\title{
Refractory inclusions with unusual chemical compositions from the Vigarano carbonaceous chondrite
}

\author{
Paul J. Sylvester, ${ }^{1}$ Lawrence Grossman ${ }^{1, *}$ and Glenn J. MacPherson ${ }^{2}$ \\ ${ }^{1}$ Department of the Geophysical Sciences, The University of Chicago, 5734 South Ellis Avenue, Chicago, IL 60637, USA \\ ${ }^{2}$ Department of Mineral Sciences, National Museum of Natural History, Smithsonian Institution, Washington, DC 20560, USA
}

(Received July 15, 1991; accepted in revised form December 11, 1991)

\begin{abstract}
Ten inclusions, nine from Vigarano and one from Leoville, both members of the reduced subgroup of $\mathrm{C} 3 \mathrm{~V}$ chondrites, were analyzed for major and trace elements by neutron activation. Most have some refractory element characteristics that are common in refractory inclusions from Allende, a member of the oxidized subgroup of C3V chondrites. For instance, three (Leo 3537-2, Vig 1623-8, Vig 1623-11) are Type B inclusions with Group I or modified Group I REE patterns, three (Vig 1623-5, Vig 1623-10, Vig 1623-13) are olivine-rich inclusions, each of which is rather uniformly diluted in most refractory elements compared to Group I inclusions, and two (Vig 1623-14, Vig 1623-16) are fine-grained inclusions with Group II REE patterns. Six of the Vigarano inclusions, however, have refractory element fractionations that are unusual in Allende inclusions. Vig 1623-11 has Cl chondrite-normalized enrichment factors for Th and refractory siderophiles that are lower than those for REE by $60 \%$ or more, a positive Mo anomaly $(\mathrm{Mo} / \mathrm{Ir}=1.92 \pm 0.02$ relative to $\mathrm{Cl}$ chondrites) and an unusually low $\mathrm{Zr} / \mathrm{Hf}$ ratio $(0.67$ \pm 0.06 relative to $\mathrm{Cl}$ ). Relative to $\mathrm{C} 1$ chondrites, the $\mathrm{Ir}$ enrichment factor is 1.7 times greater than the mean enrichment factor for Re, Os, and Ru in Vig 1623-10. Vig 1623-13 has one of the lowest Ir/Sm ratios $(0.00044 \pm 0.00007$ relative to $\mathrm{C1})$ ever measured in an olivine chondrule. Vig 1623-16 has a modified Group II REE pattern which reflects removal of refractory REEs from the nebular gas at unusually low temperatures. Vig 477-5 has a REE pattern with a small negative $\mathrm{Ce}$ anomaly $(\mathrm{Ce} / \mathrm{La}=0.79 \pm 0.01$ relative to $\mathrm{C1}$ ), a large negative $\mathrm{Yb}$ anomaly $(\mathrm{Yb} / \mathrm{Lu}=0.57 \pm 0.02$ relative to $\mathrm{C1}$ ) and no Eu anomaly. This inclusion also has a positive Mo anomaly $(\mathrm{Mo} / \mathrm{Ir}=2.25 \pm 0.03$ relative to $\mathrm{C} 1)$ and enrichment factors for $\mathrm{Zr}$ and $\mathrm{Th}$ that are lower than those for $\mathrm{Lu}$ by about 65\%. Vig 477-B has a REE pattern that may be a mixture of five parts of a component with an ultrarefractory REE pattern and ninety-five parts of another with a modified Group II REE pattern. Vigarano apparently sampled a different population of refractory inclusions from Allende, presumably because refractory nebular materials were not well mixed where and when $\mathrm{C} 3 \mathrm{~V}$ chondrites accreted. The Vigarano and Leoville coarse-grained inclusions studied here, like others analyzed previously, tend to have lower concentrations of $\mathrm{Na}$ and $\mathrm{Au}$ than Allende coarse-grained inclusions, but the two Vigarano fine-grained inclusions of this study have concentrations of these elements that are comparable to those of Allende fine-grained inclusions. Thus, although coarse-grained inclusions in the reduced subgroup of $\mathrm{C} 3 \mathrm{~V}$ chondrites were altered under different conditions from those in the oxidized subgroup, some fine-grained inclusions in the reduced subgroup were altered under similar conditions as some in the oxidized subgroup.
\end{abstract}

\section{INTRODUCTION}

REFRACTORY, Ca-, Al-RICH inclusions (CAIs) in carbonaceous chondrites are thought to be early, high-temperature condensates from a cooling solar nebular gas, modified by later partial melting, evaporation, and low-temperature alteration. Recently, MAO et al. (1990) analyzed the bulk compositions of five CAIs from Leoville and Vigarano, members of the reduced subgroup of C3V chondrites (MCSWEEN, 1977). Three have refractory element characteristics that are rare in CAls from Allende, a member of the oxidized subgroup of C3V chondrites (MCSWEEN, 1977). They suggested, therefore, that Allende inclusions (those for which the most data exist) are not representative of all early nebular condensates that accreted into $\mathrm{C} 3 \mathrm{~V}$ chondrites, and that Vigarano and Leoville are more likely than Allende to contain refractory inclusions that record undiscovered nebular frac-

* Also Enrico Fermi Institute, University of Chicago, 5640 South Ellis Avenue, Chicago, IL 60637, USA. tionations. Here, we determine the chemical compositions of nine additional Vigarano inclusions and one from Leoville by instrumental neutron activation analysis (INAA) in order to test the MAO et al. (1990) hypothesis. The idea that there are different $\mathrm{CAI}$ populations in different $\mathrm{C} 3 \mathrm{~V}$ chondrites has also been suggested by LIU and SCHMITT (1988). It is important to determine if this is true, as it would imply that early condensates were distributed heterogeneously in the nebular region where and when $\mathrm{C} 3 \mathrm{~V}$ chondrites accreted.

Petrographic and/or isotopic data have been reported for seven of the ten samples analyzed here. CAILLET et al. (1991) determined the $\mathrm{Mg}$ isotopic composition of anorthite in Leo 3537-2. The mineralogy and petrography of Vig 477-B were described by CAILLET et al. (1988). In the same inclusion, ZINNER et al. (1989) measured $\mathrm{O}$ and $\mathrm{Mg}$ isotopic compositions of periclase, spinel, and melilite, and EL GORESY et al. (1990a) determined $\mathrm{Mg}$ isotopic compositions of spinel, melilite, and hibonite. The mineralogy and petrography of Vig 477-5 were described by MACPHERSON (1985). Concentrations of rare earth elements in fassaite and perovskite from 
Vig 477-5 were measured by DAVIS et al. (1986), and in melilite by DAvis et al. (1987). Magnesium isotopic analyses of spinel and melilite in that inclusion were reported by DAVIS et al. (1986), and of melilite and hibonite by DAVIS et al. (1987). The chemical data for Vig 1623-5 discussed herein were presented initially as part of a detailed petrographic and $\mathrm{Mg}, \mathrm{Si}$, and $\mathrm{O}$ isotopic study by DAvis et al. (1991). $\mathrm{Mg}, \mathrm{Ca}$, $\mathrm{Ti}, \mathrm{Sr}, \mathrm{Sm}$, and $\mathrm{Nd}$ isotopic data were determined for $\mathrm{Vig}$ 1623-5 by Loss et al. (1990a); its Cr isotopic composition was reported by Loss et al. (1990b). For Vig 1623-8, ClAYTON et al. (1987) measured the $\mathrm{O}$ and $\mathrm{Si}$ isotopic compositions of the bulk inclusion, Loss et al. (1990a) determined the $\mathrm{Mg}$, $\mathrm{Ca}$, and $\mathrm{Ti}$ isotopic compositions of the bulk inclusion, and MACPherson and DAvIS (1990) measured Mg isotopic compositions of anorthite, melilite, and fassaite. CLAYTON et al. (1987) measured the $O$ isotopic composition of Vig 1623-14 and the $\mathrm{O}$ and $\mathrm{Si}$ isotopic compositions of Vig 162313. Preliminary results of the present study were presented by SYLVESTER et al. (1990a). In the latter paper, a chemical analysis of Vigarano inclusion V $1 \mathrm{c}$ was reported, but the material analyzed is probably not from VIc and, thus, that analysis is not included here.

\section{SAMPLE DESCRIPTIONS}

A sawn slab of Leoville and a piece of a single Vigarano stone from the Smithsonian Institution were used in this study. A diamond saw was used to cut four slices, each $\sim 4 \times 5 \mathrm{~cm}$, from the Vigarano stone, and all inclusion samples studied here were dug from cut surfaces of these two meteorites using stainless steel dental tools and a micro-trimmer in a clean room, except for Vig 1623-5 which was sampled from the rough edge of a slab. A portion of each inclusion was made into a polished thin section from which a point-count mode was determined, except for the two fine-grained inclusions, Vig 1623-14 and Vig 1623-16, in which the phases are too finely intergrown for accurate modal determinations. Results are shown in Table 1 where they are also compared with modes calculated from bulk compositions determined in the present work.

\section{Leoville 3537-2}

This is a spherical Type $\mathrm{B} 1$ inclusion, $\sim 1.1 \mathrm{~cm}$ in diameter, consisting of melilite, anorthite, fassaite, spinel, and minor NiFe metal.
Relative to other Type B1 inclusions, Jeo $3537-2$ has an unusually pronounced zonal distribution of mineral phases. The outer 0.4-1.6 $\mathrm{mm}$ (mantle) is melilite-rich and contains only minor amounts of other phases. Interior to this is a $0.8-1.2 \mathrm{~mm}$ thick, fassaite-rich zone (outer core), and the center (inner core) of the inclusion is spineland anorthite-rich. Melilite in the mantle is Al-rich near the inclusion rim and $\mathrm{Mg}$-rich away from it, and fassaite in the outer core is $\mathrm{Ti}$ and Al-rich near the outer core/mantle boundary and becomes more Ti- and Al-poor toward the center of the inclusion. The inclusion contains minor secondary alteration products, mainly veinlets of calcite and an unknown, Si-rich, Al-bearing phase that gives $\sim 85 \%$ analytical sums with an electron microprobe. There is a WARK-LOVERING (1977) rim sequence consisting, from innermost to outermost, of hibonite or perovskite, spinel + gehlenitic melilite, diopside, and forsteritic olivine. Melilite is $\mathrm{Ă}_{10-74}$, with up to $\sim 0.22 \% \mathrm{Na}_{2} \mathrm{O}$ which correlates positively with Âk content only for melilite having $\hat{A} k$ $>\sim 40$. The most gehlenitic melilite is relatively rare and located adjacent to the rim sequence. Most mantle melilite is $\AA \mathrm{k}_{20-40}$. Fassaite contains $13-25 \% \mathrm{Al}_{2} \mathrm{O}_{3}$ and $2-11 \% \mathrm{TiO}_{2}$, with $\leq 0.23 \% \mathrm{FeO}$ and $\leq 0.45 \% \quad \mathrm{~V}_{2} \mathrm{O}_{3}$. Anorthite is $\mathrm{An}_{99-100}$, containing $\leq 0.14 \% \quad \mathrm{Na}_{2} \mathrm{O}$, $\leq 0.54 \% \mathrm{FeO}$ and $\leq 0.18 \% \mathrm{MgO}$.

\section{Vigarano 477-B}

This is a Type $\mathrm{B} 1$ inclusion, approximately elliptical in shape and $4 \times 6 \mathrm{~mm}$ in size, with a $0.3-0.8 \mathrm{~mm}$ thick, melilite-rich mantle. Vig 477-B is enclosed in a WARK-LOVERING (1977) rim sequence consisting, from outermost to innermost, of olivine, diopside, Febearing spinel + perovskite + hibonite, and feldspathoids. Unlike textures found in some Type B1 inclusion mantles, many melilite crystals in the mantle of Vig 477-B have scalloped grain-grain boundaries, rather than being euhedral to subhedral laths, and few are oriented at high angles to the inclusion margin. The most Al-rich melilite is near the rim sequence (regardless of crystal orientation), along with small blades of hibonite. Spinel grains in the mantle are less abundant and smaller in size than those in the inclusion core. The core is dominated by abundant, large (up to $\sim 1 \mathrm{~mm}$ ) fassaite crystals, lesser melilite, and sparse anorthite, all enclosing abundant spinel grains and rounded grains of Ni-Fe metal (kamacite and taenite) + phosphate. Fassaite crystals are zoned and range in color from pleochroic green to colorless, the most intensely colored parts of the crystals being found close to the core-mantle interface. Prominent spinelfree islands are up to $\sim 2.4 \mathrm{~mm}$ in size and consist almost entirely of melilite with minor fassaite. The melilite occurs as a dense intergrowth of well-formed laths up to $0.2 \mathrm{~mm}$ in size, much smaller than the near-mm-sized melilite grains elsewhere in the inclusion. The laths commonly show concentric and oscillatory zoning. The inclusion is little altered. A $\sim 10-20 \mu \mathrm{m}$ thick zone near the rim contains

Table 1. Comparison of observed and calculated ${ }^{1}$ modes for inclusions studied in this work.

\begin{tabular}{|c|c|c|c|c|c|c|c|c|c|}
\hline Sample & Method & Melilite & Fassaite & Spinel & Olivine & Anorthite & Perovskite & Sodalite & Hibonit \\
\hline Leo 3537-2 & $\begin{array}{l}\text { calc } \\
\text { obs }\end{array}$ & $\begin{array}{l}42 \\
48\end{array}$ & $\begin{array}{l}21 \\
27\end{array}$ & $\begin{array}{l}28 \\
16\end{array}$ & & $\begin{array}{l}8 \\
9\end{array}$ & & & \\
\hline Vig 477-B & $\begin{array}{l}\text { calc } \\
\text { obs }\end{array}$ & $\begin{array}{l}40 \\
65\end{array}$ & $\begin{array}{l}22 \\
12\end{array}$ & $\begin{array}{l}38 \\
20\end{array}$ & & 2 & & & \\
\hline Vig 477-5 & $\begin{array}{l}\text { calc } \\
\text { obs }\end{array}$ & $\begin{array}{l}91 \\
90\end{array}$ & & $\begin{array}{l}1 \\
2\end{array}$ & & & $\begin{array}{l}2 \\
2\end{array}$ & & $\begin{array}{l}6 \\
6\end{array}$ \\
\hline Vig 1623-5 & $\begin{array}{l}\text { calc } \\
\text { obs }\end{array}$ & $\begin{array}{l}11 \\
26\end{array}$ & $\begin{array}{l}17 \\
16\end{array}$ & $\begin{array}{l}2 \\
7\end{array}$ & $\begin{array}{l}67 \\
52\end{array}$ & & & & \\
\hline Vig $1623-8$ & $\begin{array}{l}\text { calc } \\
\text { obs }\end{array}$ & $\begin{array}{l}50 \\
52\end{array}$ & $\begin{array}{l}18 \\
20\end{array}$ & $\begin{array}{l}23 \\
10\end{array}$ & & $\begin{array}{r}7 \\
18\end{array}$ & & & \\
\hline Vig $1623-10$ & $\begin{array}{l}\text { calc } \\
\text { obs }\end{array}$ & & $\begin{array}{r}5 \\
13\end{array}$ & 1 & $\begin{array}{l}80 \\
75\end{array}$ & $\begin{array}{r}13 \\
5\end{array}$ & & $\begin{array}{l}1 \\
4\end{array}$ & \\
\hline Vig $1623-11$ & $\begin{array}{l}\text { calc } \\
\text { obs }\end{array}$ & $\begin{array}{l}68 \\
67\end{array}$ & $\begin{array}{l}20 \\
16\end{array}$ & $\begin{array}{r}10 \\
7\end{array}$ & & $\begin{array}{r}1 \\
11\end{array}$ & & & \\
\hline Vig $1623-13$ & $\begin{array}{l}\text { calc } \\
\text { obs }\end{array}$ & & $\begin{array}{l}24 \\
20\end{array}$ & $\begin{array}{l}11 \\
13\end{array}$ & $\begin{array}{l}50 \\
58\end{array}$ & $\begin{array}{r}14 \\
9\end{array}$ & & & \\
\hline
\end{tabular}

${ }^{1}$ Weight per cent 
feldspathoids, and calcite occurs along fractures and in some voids. Microprobe analyses give the following ranges: melilite, $\AA \mathrm{k}_{0}$ to $\AA \mathrm{k}_{65}$ (average composition $\sim \AA^{2} k_{28}$ ), $\leq 0.24 \% \mathrm{Na}_{2} \mathrm{O}$; fassaite, $16-21 \% \mathrm{Al}_{2} \mathrm{O}_{3}$ and $6.5-14.5 \% \mathrm{TiO}_{2}$; spinel, $\mathrm{FeO}$ mostly $0.1-0.8 \%$ with rare grains up to $12.2 \%, \mathrm{~V}_{2} \mathrm{O}_{3}$ mostly $0.4-0.8 \%$ but as high as $\sim 15 \%$, and $\mathrm{Cr}_{2} \mathrm{O}_{3}$ mostly $0.1-0.4 \%$ with a maximum of $8.8 \%$.

\section{Vigarano 477-5}

This is an irregularly shaped inclusion, roughly $1.2 \mathrm{~cm}$ in maximum dimension, that consists mostly of melilite with accessory spinel, hibonite, perovskite, and a small amount of fassaite $\left(5.8-21.9 \% \mathrm{TiO}_{2}\right)$. It is similar to "fluffy" Type A inclusions in Allende (MACPHERSON and GROSSMAN, 1984), except that, unlike the latter in which interstices between highly altered melilite crystals are filled with secondary minerals, melilite in Vig 477-5 is mostly unaltered and the crystals form a dense, polygonal-granular mosaic, suggestive of solid-state recrystallization. The inclusion is brecciated in places, with interstices filled by many tiny, broken fragments of melilite, on some of which are thin layers of secondary anorthite and pyroxene. The inclusion has a concentric distribution of phases. The irregularly shaped core is an intergrowth of hibonite blades and spinel with melilite. Surrounding the core is the melilite-rich bulk of the inclusion. DAVIS et al. (1987) used X-ray mapping to discern that the melitite was recrystallized after an earlier episode of brecciation: angular and irregular regions of polygonal-granular melilite crystals (cores of Å $k_{R-1 R}$, margins of $\AA \mathrm{k}_{2-4}$ ) are set in a uniform matrix of $\tilde{A} \mathrm{k}_{10-1}$. Most hibonite is Tiand $\mathrm{Mg}$-rich, containing up to $-4.2 \% \mathrm{MgO}$ and $8.1 \% \mathrm{TiO}_{2}$, but the cores of some crystals have $\sim 0.4 \% \mathrm{MgO}$ and $0.4 \% \mathrm{TiO}_{2}$. The rim sequence on this inclusion consists of, from innermost to outermost, spinel + perovskite, melilite, anorthite, and aluminous diopside.

\section{Vigarano 1623-5}

In slab surface, Vig 1623-5 is an oblong inclusion, roughly $4 \times 5$ $\mathrm{mm}$ in size. The thin section shows two major pieces, separated by $150-250 \mu \mathrm{m}$ of matrix; many smaller fragments occur in the matrix adjacent to both pieces. The largest piece of Vig 1623-5 has a large, nearly spherical re-entrant filled with meteorite matrix, very similar to one in the Allende inclusion ALVIN and interpreted as a vesicle by MACPHERSON et al. (1981). The interior of Vig 1623-5 is olivineand pyroxene-rich, and the exterior melilite- and spinel-rich. The two zones appear to be grossly out of equilibrium (DAVIS et al., 1991). The interior consists largely of equant, euhedral to subhedral, $30-$ $170 \mu \mathrm{m}$ olivine crystals, poikilitically enclosed within $200-500 \mu \mathrm{m}$ fassaite and åkermanitic melilite grains. Minor spinel $(5-20 \mu \mathrm{m})$ is enclosed in all other phases. Fassaite and melilite are inhomogeneously distributed. In the larger portion of Vig 1623-5, fassaite is more abundant than melilite but, in the smaller portion, there is little fassaite. Spinel crystallized first, then olivine and finally fassaite and melilite in uncertain order. Fassaite contains $9.5-22.5 \% \mathrm{Al}_{2} \mathrm{O}_{3}$ and $2-8 \% \mathrm{TiO}_{2}$. Olivine is nearly pure forsterite except for $\sim 1.7 \% \mathrm{CaO}$. Melilite in the core is uniformly $\AA k_{89}$. Overlying the core is an $\sim 200 \mu \mathrm{m}$ thick, discontinuous, melilite-rich mantle that occurs in two distinct types, depending on whether the underlying core is melilite- or fassaiterich. Over melilite-rich core, the mantle consists of melilite that grades abruptly from $\AA \mathrm{k}_{89}$ in the core to $\sim \AA \mathrm{k}_{47}$ at the inclusion margin. Spinel grains and rare hibonite crystals occur within the melilite. No olivine is found in this mantle, but there is a $50 \mu \mathrm{m}$-wide band near the core/mantle boundary where olivine is pseudomorphically replaced by nearly pure åkermanite which encloses tiny, randomly dispersed spinel grains, just as interior olivine does. Mantle overlying fassaite-rich core is characterized by a discontinuous band, 10-25 $\mu \mathrm{m}$ thick and in contact with, embaying and replacing fassaite, that consists of a symplectic intergrowth of melilite plus fine-grained vermiform perovskite. Outside both types of mantle, in contact with the meteorite matrix, is a porous band up to $100 \mu \mathrm{m}$ thick that consists of $<1-5 \mu \mathrm{m}$ grains of melilite $\left(\AA \mathrm{k}_{16-43}\right)+$ spinel + either hibonite (outside of mantle overlying melilite-rich core) or perovskite (outside of mantle overlying fassaite-rich core). Alteration effects are relatively minor in Vig 1623-5. Iron and sodium are enriched in major phases near the inclusion margin and along fractures. Veins of calcite and an unknown, silica-rich phase that preferentially replaces åkermanitic melilite are present.

\section{Vigarano 1623-8}

In slab surface, $\mathrm{Vig} 1623-8$ has a maximum diameter of 5-6 mm and varies from rounded to irregular in shape through several successive saw cuts. In thin section, it is a typical Type B2 inclusion (WARK and LOVERING, 1982), consisting of melilite, fassaite, anorthite, spinel, and minor nickel-iron metal. A WARK-LOVERING (1977) rim sequence, consisting of aluminous diopside, spinel and forsteritic olivine, is nearly continuous except along one edge where the inclusion is broken. Minor aluminous diopside is present as a secondary alteration product. Melilite ( $\left(\mathrm{Ak}_{12-72}\right.$ ) occurs as large (up to $770 \mu \mathrm{m}$ ), irregular to tabular crystals and, within about $140-210 \mu \mathrm{m}$ of the rim, polygonal-granular mosaics of $<70 \mu \mathrm{m}$-sized crystals. Melilite is concentrated toward the outside of the inclusion, but not to the degree seen in Type Bl inclusions. Most larger melilite crystals show normal concentric zoning which is partially obscured by brecciation and kink-banding. Most melilite crystals contain many small inclusions of pyroxene plus lesser spinel and anorthite. Many of the pyroxene inclusions are surrounded by narrow zones of $\mathrm{Mg}$-rich melilite, giving the host melilite crystals an overall patchy appearance that further masks the concentric zoning. The most gehlenite-rich melilite grains are found within $\sim 40-50 \mu \mathrm{m}$ or so of the WARK-LOVERING (1977) rim. Melilite crystals contain up to $0.3 \mathrm{wt} \% \mathrm{Na}_{2} \mathrm{O}$, higher than generally seen in other refractory inclusions. A strong correlation between åkermanite content and $\mathrm{Na}_{2} \mathrm{O}$ suggests that sodium was introduced during igneous crystallization. Fassaite $\left(17-23 \% \mathrm{Al}_{2} \mathrm{O}_{3}\right.$, $5-17 \% \mathrm{TiO}_{2}$ ) forms large (up to $\sim 280-350 \mu \mathrm{m}$ ), pale green to bluishgreen pleochroic crystals, with cores that are more intensely colored and higher in $\mathrm{Ti}$ and $\mathrm{Al}$ than their margins. Small fassaite grains enclosed within melilite near the outer margins of the inclusion are more intensely colored and far more $\mathrm{Ti}$ - and $\mathrm{V}$-rich (up to $17 \%$ and $0.8 \%$, respectively) than larger grains deeper inside the inclusion. Anorthite crystals are up to $390 \mu \mathrm{m}$ in size, irregular in shape and fill interstices between melilite and pyroxene. In many places, contacts between crystals of anorthite and melilite are irregular, scalloped or cuspate. Some spinel grains enclosed within anorthite have thin (a few $\mu \mathrm{m}$ or less) rims of melilite around them which, in places, are contiguous with large melilite crystals external to the anorthite, similar to textures described in several Allende inclusions by PODOSEK et al. (1991). Such features suggest anorthite is replacing melilite, but these are not the very fine-grained secondary anorthite grains of the kind commonly seen replacing melilite in Allende inclusions. Rather, these are large, coarsely twinned, single crystals. Nepheline occurs as lamellae within anorthite rather than around grain margins, its typical mode of occurrence as an alteration product. Spinel is very heterogeneously distributed, being concentrated within the core and relatively deficient in the outer $\sim 1 \mathrm{~mm}$. In addition, spinel grains are heavily concentrated within anorthite and fassaite crystals and are far less abundant within melilite.

\section{Vigarano 1623-10}

This white inclusion is irregular in shape and $\sim 1.5 \times 3.5 \mathrm{~mm}$ in maximum size. In thin section, Vig 1623-10 is an amoeboid olivine inclusion like those described by GROSSMAN and STEELE (1976) in Allende. The outer parts of the inclusion consist mostly of bulbous masses of olivine with an equigranular texture, separated by meteorite matrix. The olivine clumps surround a more coherent inclusion core that also consists mostly of olivine. In the centers of the olivine clumps, and scattered within the inclusion interior, are irregular areas consisting of feldspathoids, anorthite, aluminous diopside and ironchromium-rich spinel $\left(\sim 18 \% \mathrm{FeO},>2 \% \mathrm{Cr}_{2} \mathrm{O}_{3}\right)$. Generally, the spinel grains are in the centers of these aluminum-rich areas, completely enclosed by feldspathoids + anorthite. A boundary layer of diopside $\left(2-15 \% \mathrm{Al}_{2} \mathrm{O}_{3},<1-7 \% \mathrm{TiO}_{2}\right.$ ) frequently separates the interiors of the aluminum-rich areas from the olivine. Olivine in the interior of Vig 1623-10 is mostly Fo $_{90-100}$. Near the outer parts of the inclusion, however, olivine crystals have iron-rich rims (up to $\mathrm{Fo}_{72}$ ) that parallel grain boundaries and fracture traces. These Fe-rich rims presumably formed by reaction of the $\mathrm{Mg}$-rich cores with an Fe-rich vapor. A 
solid solution consisting of $63 \%$ kirschsteinite $\left(\mathrm{CaFeSiO}_{4}\right)$ and $37 \%$ monticellite $\left(\mathrm{CaMgSiO}_{4}\right)$ is found as sparse grains. Opaque assemblages consisting of $\mathrm{Ni}-\mathrm{Fe}$ metal $(\sim 44 \% \mathrm{Ni})$, troilite, and iron oxide are found as accessories.

\section{Vigarano 1623-11}

This is an oblong inclusion, $\sim 2 \times 4 \mathrm{~mm}$ in size, consisting mostly of melilite with lesser fassaite, anorthite and spinel. Although Vig 1623-11 is predominantly melilite, a characteristic of Type A inclusions, the presence of coarse anorthite and more than trace amounts of fassaite suggests it is a Type B. Minor nepheline and calcite near the rim sequence are the only petrographic evidence of secondary alteration. Most melilite forms dense, polygonal-granular mosaics, except where large, spongy, poikilitic fassaite grains enclose small, euhedral melilite tablets. Similar euhedral melilite tablets are enclosed within anastamosing veinlets of anorthite. Some spinel grains are heterogeneously distributed throughout melilite but, like the anorthite, most spinel is in the anorthite veinlets. In fact, in many places, spinel grains occur exclusively within veinlets and do not extend beyond the veinlet walls. Overall, Vig 1623-11 appears to be a melilite-rich inclusion that was intruded by melt veins from which anorthite and spinel crystallized. Melilite is $\AA k_{17-45}$, with an average of $\sim \AA \mathrm{k}_{25}$. Minor element contents are $\mathrm{Na}_{2} \mathrm{O} \leq 0.08 \%$ and $\mathrm{TiO}_{2} 0.04-0.09 \%$; $\mathrm{K}_{2} \mathrm{O}$ was not detected. Fassaite has $16-23 \% \mathrm{Al}_{2} \mathrm{O}_{3}, 6-12 \% \mathrm{TiO}_{2}$, $\leq 0.32 \% \mathrm{FeO}$, and $\leq 0.38 \% \mathrm{~V}_{2} \mathrm{O}_{3}$. Anorthite is $\mathrm{An}_{99-100}$, with $\leq 0.08 \%$ $\mathrm{FeO}, \leq 0.21 \% \mathrm{MgO}, \leq 0.03 \% \mathrm{Na}_{2} \mathrm{O}$, and $\leq 0.1 \% \mathrm{TiO}_{2}$. The chief minor components in spinel are $\mathrm{TiO}_{2}$ (up to $0.44 \%$ ), $\mathrm{V}_{2} \mathrm{O}_{3}$ (up to $0.23 \%$ ), and $\mathrm{CaO}(\sim 0.18 \%)$.

\section{Vigarano 1623-13}

This $-3 \times 4 \mathrm{~mm}$, oblong, white object appears in slab surface to be a typical refractory inclusion, but thin section study reveals that it is a very large, Al-rich, barred olivine chondrule. Its shape varies from elliptical to irregular from one slab to another. Long, bent and somewhat dendritic bars of olivine $\left(\mathrm{Fo}_{0_{3-96}}\right)$ enclose equant granules of spinel $\left(2.2-5.5 \% \mathrm{FeO}, 3.8-4.6 \% \mathrm{Cr}_{2} \mathrm{O}_{3}\right)$. Interstices between olivine bars consist of spinel, anorthite $\left(\sim \mathrm{An}_{92}\right)$ and fassaite $\left(\sim 14 \% \mathrm{Al}_{2} \mathrm{O}_{3}\right.$, $2 \% \mathrm{TiO}_{2}, 0.7 \% \mathrm{Cr}_{2} \mathrm{O}_{3}$ ). Major phases show only slight compositional zoning.

\section{Vigarano 1623-14}

This is a very fine-grained, spinel-rich inclusion, $\sim 1.8 \times 2.4 \mathrm{~mm}$ in size. It is crudely elliptical to rectangular in shape, with embayments along the margin. The main constituents are Fe-bearing spinel, pyroxene, anorthite, nepheline, and sodalite. It differs from Vigarano coarse-grained inclusions in containing abundant feldspathoids. The inclusion has a concentrically zoned mineral distribution, with the pinkish core being more spinel- and feldspathoid-rich and the outer, gray-white part being more pyroxene- and anorthite-rich. An approximate mode for Vig 1623-14, obtained by point-counting a backscattered electron photomosaic of the thin section, yields $\sim 8 \%$ spinel, $19 \%$ pyroxene, and $74 \%$ anorthite + feldspathoids. Because the thin section contains mostly material from the core, the mode probably underestimates anorthite and pyroxene. Similar to spinel-rich, finegrained inclusions in Allende, spinel grains in Vig 1623-14 are concentrically mantled: pale pink spinel cores $(1-13 \% \mathrm{FeO})$ are enclosed within a $<5 \mu \mathrm{m}$ rim of feldspathoids + anorthite, followed by an outer, $\sim 1 \mu \mathrm{m}$ rim of fassaite (up to $\sim 23 \% \mathrm{Al}_{2} \mathrm{O}_{3}, 12 \% \mathrm{TiO} \mathrm{O}_{2}$ ). Within the feldspathoids + anorthite layer, sodalite and nepheline surround ragged patches of anorthite in an apparent replacement texture. Interstitial to the rimmed spinel grains in the core of Vig 1623-14 are extensive regions of feldspathoids, Fe-rich spinel (9-13\% FeO), anorthite that again appears to be enclosed in and partially replaced by feldspathoids, pyroxene, and rare grains of kirschsteinite-rich monticellite $\left(\mathrm{Kir}_{41-49} \mathrm{Mont} \mathrm{f}_{5-51}\right)$. The outer zone of Vig 1623-14 contains a subequal mixture of irregular coherent stringers of pyroxene and interstitial patches of anorthite, without concentrically rimmed spinel grains.

\section{Vigarano 1623-16}

This is a fine-grained, spinel-rich inclusion, diamond-shaped and $\sim 1.8 \times 2.2 \mathrm{~mm}$ in dimension. Like Vig 1623-14, Vig 1623-16 is concentrically zoned, with a pink, spinel- and feldspathoid-rich core and a lighter, pyroxene-rich mantle. The core consists of spinel, feldspathoids, fassaitic pyroxene, anorthite, forsteritic olivine, and traces of perovskite, ilmenite and an Fe-rich garnet. The mantle consists predominantly of diopsidic augite, spinel, melilite, and minor anorthite and feldspathoids. Spinel grains in the core of Vig 1623-16 are concentrically rimmed: pale pink spinel cores (up to $\sim 10.6 \%$ $\mathrm{FeO}$ ) are enclosed within a $<5 \mu \mathrm{m}$ rim of feldspathoids + anorthite, followed by an outer, $-1 \mu \mathrm{m}$ rim of fassaite $\left(\mathrm{TiO}_{2}\right.$ up to $\left.8 \%\right)$. Perovskite grains are scattered within many of the spinel crystals. Interstitial to the rimmed spinel grains in the core of Vig 1623-16 is a porous mixture of feldspathoids, olivine (Fogs), anorthite, diopside, and occasional grains of ilmenite and kirschsteinite-rich monticellite $\left(\sim \mathrm{Kir}_{29} \mathrm{Mont}_{71}\right)$. The compact mantle of $\mathrm{Vig} 1623-16$ is quite different from that of Vig 1623-14: it consists mostly of densely intergrown diopsidic augite crystals that locally enclose subordinate patches of melilite $\left(\AA \mathrm{K}_{27-36}\right)$ and $\mathrm{Fe}$-poor spinel ( $<0.5 \% \mathrm{FeO} ; \mathrm{Cr}_{2} \mathrm{O}_{3} \sim 0.6 \%$ ). Small amounts of anorthite and feldspathoids are also present. Some spinel grains near the edge of the inclusion are deep pink to purple, contain up to $22 \% \mathrm{FeO}$ and $\sim 1.5 \% \mathrm{Cr}_{2} \mathrm{O}_{3}$, and enclose tiny grains of ilmenite.

\section{EXPERIMENTAL METHODS}

\section{Rabbit Irradiation}

Experimental procedures and standards used for the short INAA irradiation, carried out at the University of Missouri Research Reactor (MURR), were the same as those of $D_{\text {AvIS }}$ et al. (1982), except that the neutron flux was $8 \times 10^{13} \mathrm{n} / \mathrm{cm}^{2}$-sec. Samples and standards were counted at the MURR three times: first, for $4-8 \mathrm{~min}$ on an $11.6 \%$ efficiency $\mathrm{Ge}(\mathrm{Li})$ detector, 3-10 min after irradiation; next, in a position closer to the same detector for 2-8 $\mathrm{min}, 9-46 \mathrm{~min}$ after irradiation; and lastly, on one of two $\mathrm{Ge}(\mathrm{Li})$ detectors with efficiencies of 28.1 and $35.5 \%$ for $9-30 \mathrm{~min}, 2-12 \mathrm{hr}$ after irradiation. Neutron flux corrections of $55.2 \%$ were applied to each sample and standard based on variations in the specific activity of ${ }^{60} \mathrm{Co}$, assumed to change linearly with time, in Co-doped aluminum wires irradiated during the course of the experiment. Polyethylene pouches into which samples and standards were packaged for irradiation and counting required blank corrections of $0-39 \%$ of the amount of $\mathrm{Cl}$ present in each sample. Blank corrections were $\leq 5 \%$ for all other elements determined, except in Vig 1623-13, for which corrections of $10 \%$ were made to the amounts of Dy and Ti present, due to the low concentrations of these elements in this sample; and in Vig 1623-5, for which corrections of $10-51 \%$ were made to the amounts of $\mathrm{Al}, \mathrm{V}$, $\mathrm{Ti}, \mathrm{Dy}$, and Mn present, due to the small size of this sample. Specpure standards of $\mathrm{MgO}, \mathrm{Al}_{2} \mathrm{O}_{3}$, and $\mathrm{SiO}_{2}$ were used to correct for the fast neutron reactions, ${ }^{27} \mathrm{Al}(n, p){ }^{27} \mathrm{Mg}$ and ${ }^{28} \mathrm{Si}(n, p){ }^{28} \mathrm{Al}$. INAA results are shown in Table 2 , inctuding sample sizes and the standard, nuclide, photopeak energy, and half-life used for each element. Errors quoted are $1 \sigma$ uncertainties due only to counting statistics. Upper limits are based on $2 \sigma$ uncertainties.

\section{Long Irradiation}

Experimental procedures and standards used for the long INAA irradiation were the same as those of MAO et al. (1990), with some important improvements. In order to lower blank contamination levels that had hindered our ability to determine concentrations of some elements in very small samples (MAO et al., 1991), supersilica tubes in which samples were irradiated and counted were washed with ultra-pure $\mathrm{HCl}$ and $\mathrm{HNO}_{3}$, double-distilled from Vycor glass. Also, two sets of gas line filters were used to filter the oxygen and methane gas that, when ignited, provided a flame used to seal the supersilica tubes. The pre-filter was a Cole-Parmer syringe filter consisting of a polytetrafluoroethylene (PTFE) membrane, $20 \mathrm{~cm}^{2}$ in area, with a $0.22 \mu \mathrm{m}$ filtration rating, housed in a polypropylene shell. The final filter was a Nuclepore gas line filter consisting of a 
stainless steel shell and two PTFE layers with an area of $360 \mathrm{~cm}^{2}$ for filtering particles $\geq 0.02 \mu \mathrm{m}$.

The package of sample, standard, and empty supersilica tubes was irradiated in row 1 of the graphite reflector of the MURR for $126 \mathrm{~h}$ at a flux of $8 \times 10^{13} \mathrm{n} / \mathrm{cm}^{2} / \mathrm{sec}$ and then transferred to the flux trap of the same reactor where, one day later, the irradiation continued for another $159 \mathrm{~h}$ at a flux of $4 \times 10^{14} \mathrm{n} / \mathrm{cm}^{2} / \mathrm{sec}$. After irradiation, each sample was counted four times at the University of Chicago on either of two high-purity Ge detectors with efficiencies of $35.7 \%$ and 37.9\%: for 2.3-14 h, 19-64 h after irradiation; for 8.9-33 h, 2.7-8.5 days after irradiation; for 1.7-3.1 days, 9.5-38 days after irradiation; and for 6.2-11 days, 17-60 days after irradiation.

\section{Data Reduction}

All corrections applied to the INAA data were made by procedures described in MAO et al. (1990). Corrections for the spatial variation of neutron flux of $\leq 7.5 \%$ were made to each sample and standard. Lanthanum interference on ${ }^{141} \mathrm{Ce}$ by double neutron capture on ${ }^{139} \mathrm{La}$, $\mathrm{Eu}$ interference on ${ }^{153} \mathrm{Sm}$ by double neutron capture on ${ }^{151} \mathrm{Eu}$, and $\mathrm{Yb}$ interference on ${ }^{177} \mathrm{Lu}$ by decay of reactor-produced ${ }^{177} \mathrm{Yb}$ resulted in corrections amounting to $0.3-6 \%, 0-6 \%$, and $2-7 \%$, respectively, in all samples. Corrections for the overlap of the peak of one nuclide on that of another werc based on the ratio of the area of the interfering peak to that of another peak of the same nuclide in a standard. These amounted to $4-18 \%$ for ${ }^{182} \mathrm{Ta}(84.7 \mathrm{keV})$ on ${ }^{170} \mathrm{Tm}(84.3 \mathrm{keV}), 0$ ${ }_{10 \%}$ for ${ }^{199} \mathrm{Au}(208.2 \mathrm{keV})$ on ${ }^{177} \mathrm{Lu}(208.4 \mathrm{keV}), 0-77 \%$ for ${ }^{136} \mathrm{Eu}$ $(811.6 \mathrm{keV})$ on ${ }^{58} \mathrm{Co}(810.8 \mathrm{keV}), 0-36 \%$ for ${ }^{154} \mathrm{Eu}(756.9 \mathrm{keV})$ on ${ }^{95} \mathrm{Zr}(756.7 \mathrm{keV}), 0-24 \%$ for ${ }^{169} \mathrm{Yb}(130.5 \mathrm{keV})$ on ${ }^{191} \mathrm{Os}(129.4 \mathrm{keV})$, $0-27 \%$ for ${ }^{182} \mathrm{Ta}(264.1 \mathrm{keV})$ on ${ }^{75} \mathrm{Se}(264.7 \mathrm{keV})$, and $0-70 \%$ for ${ }^{177} \mathrm{Lu}(136.5 \mathrm{keV})$ on ${ }^{186} \mathrm{Re}(137.1 \mathrm{keV})$. As a consequence of the large neutron fluence of this irradiation, the specific activity of ${ }^{152} \mathrm{Eu}$ in the samples varied from $12 \%$ less to $10 \%$ more than that of the REE standard, and corrections to the activity of ${ }^{152} \mathrm{Eu}$ in each sample were made accordingly.

Holmium was determined from ${ }^{166} \mathrm{Ho}$ using the method of KAWABE et al. (1986) to correct for Dy interference. We found that 25$53 \%$ of the total ${ }^{166} \mathrm{Ho}$ counts in all samples, except Vig 1623-16. were due to Dy, and made appropriate corrections. No correction was made to the ${ }^{166} \mathrm{Ho}$ counts of Vig 1623-16 because Dy is below detection in this sample.

\section{Blank Corrections}

Blank corrections were calculated from the mean amount of each element of interest present in four empty supersilica tubes prepared in the same way as, and irradiated along with, tubes containing the samples. These corrections were $\leq 10 \%$ of the amount present of each element in all samples with the following exceptions: $16-42 \%$ of the amounts of $\mathrm{Ta}, \mathrm{As}, \mathrm{Co}, \mathrm{Au}, \mathrm{Br}$, and $\mathrm{Zn}$ in Vig 477-5; 18-25\% of the amounts of $\mathrm{Ta}$, Th, and $\mathrm{Zn}$ in Vig $1623-5 ; 12-23 \%$ of the amounts of Ta, As, and Th in Vig 1623-10; 14 and 27\% of the amounts of Ni and $\mathrm{Ba}$, respectively, in Vig 1623-14; and 34\% of the amount of $\mathrm{W}$ in Vig 1623-11. Also, for $K$, blank corrections of $11-66 \%$ of the amount present were made for six samples. The reason that blank corrections tended to be greatest in Vig 477-5 and Vig 1623-5 is that these samples have masses that are smaller than the other samples by factors of 3.5-130.

One of the empty supersilica tubes has a $W$ concentration that is ten times higher than the mean $\mathrm{W}$ concentration in the other three tubes. Because of this, we report $W$ concentrations, uncorrected for a blank, as upper limits in Table 2 whenever the $\mathrm{Cl}$ chondrite-normalized concentration of $W$ is greater than the mean of those of $R e$, Os, Ir, Mo, and Ru. This is the case for Vig 1623-5, Vig 1623-10, and Vig 1623-16 in which the $\mathrm{Cl}$ chondrite-normalized concentrations of $W$ would have been $9.6,46$, and $>14$ times higher, respectively, than the means of those of the other siderophiles. Blank corrections for $\mathrm{W}$ in the remaining samples were made by using the mean $W$ concentration in the three empty tubes that do not have the anomalously high $\mathbf{W}$ content.

Blank concentrations for many elements determined in this work are lower than those of the same elements in other recent studies performed in this laboratory. Silica tubes in the latter were made and sealed with a flame fueled by unfiltered gases and were washed with reagent grade acids. In contrast, in this work, filtered gases and ultrapure acids were used. MAO et al. (1991) reported mean element levels for empty tubes prepared in the former way, and these are compared to those of this study in Table 3. Some means are reported as upper limits with standard deviations because some of the individual determinations from which they are computed are upper limits that are treated as actual values. The mean blank levels of this study are significantly smaller than those of MAO et al. (1991) for Na (by 65\%), $\mathrm{Sc}(>91 \%), \mathrm{Cr}(83 \%), \mathrm{Co}(94 \%), \mathrm{Ni}(98 \%), \mathrm{La}(96 \%), \mathrm{Ce}(76 \%), \mathrm{Sm}$ $(76 \%)$, and $\mathrm{Au}(>41 \%)$. We suspect that much of the reduction is due to use of filtered gases and ultra-pure acids; but, since the tubing in the two experiments was fashioned from different batches of supersilica, we cannot rule out the possibility that supersilica used in the present experiment originally had lower element concentrations than that used by MAO et al. (1991).

\section{Filomena}

We included a small aliquot $(168 \mu \mathrm{g})$ of the IIAB hexahedrite, Filomena, in the long irradiation as a secondary standard. Concentrations of $\mathrm{Cr}, \mathrm{Co}, \mathrm{Ni}, \mathrm{Ga}, \mathrm{As}, \mathrm{W}, \mathrm{Re}, \mathrm{Os}, \mathrm{Ir}, \mathrm{Ru}$, and $\mathrm{Au}$ determined here are within $2 \sigma$ of the means of these elements in replicate analyses by PERNICKA and WASSON (1987) and WASSON et al. (1989). Except for $\mathrm{Cr}, \mathrm{Ni}$, and $\mathrm{Ir}$, the concentration of each element in Filomena determined here is also within $2 \sigma$ of that determined previously in this laboratory, using a $1.4 \mathrm{mg}$ sample (SYLVESTER et al., 1990b). These results indicate that, even for $\mu \mathrm{g}$-sized samples, heterogeneities in Filomena are smaller than the uncertainties of INAA, except for $\mathrm{Cr}, \mathrm{Ni}$, and $\mathrm{Ir}$. While $\mathrm{Ni}$ and $\mathrm{Ir}$ concentrations differ from our previous determinations by amounts that lie just beyond analytical uncertainty, $\mathrm{Cr}$ concentrations are grossly different. For $\mathrm{Cr}$, WASsON et al. (1989) suggested that the large standard deviation of their mean (14\%) reflected nonrepresentative sampling of daubreelite. This may be the reason for the discrepancy between the $\mathrm{Cr}$ concentrations in the $\mu \mathrm{g}$ and $\mathrm{mg}$-sized aliquots of Filomena analyzed in this laboratory.

\section{MINERAL MODES CALCULATED FROM MAJOR ELEMENT CONCENTRATIONS}

Silica is the only major constituent of $\mathrm{Ca}$-, Al-rich inclusions not determined in this work, so computation of silica by difference is a very good approximation. We can use electron microprobe analyses of the phases studied in a polished thin section of a split of each inclusion and our INAA determination of the bulk chemical composition of each to calculate a modal mineralogical composition. These are compared in Table 1 with actual point-count modes determined in thin sections of eight of the inclusions analyzed by INAA. Poor agreement between calculated and observed modes may indicate that the sample taken for INAA is mineralogically nonrepresentative of the bulk inclusion.

Calculated and observed modes are in very good agreement with one another for Vig 477-5, Vig 1623-10, and Vig 1623-13. The samples of Vig 1623-8 and Leo 3537-2 taken for INAA are richer in spinel and poorer in other phases than their counterparts seen in thin section. The sample of Vig 1623-11 used for INAA is much poorer in anorthite than the one in thin section. A large discrepancy exists between calculated and observed modes for Vig 477-B. The thin section is much richer in melilite and much poorer in tassaite and spinel than the sample studied by INAA, probably because this inclusion is unusually heterogeneous in mineralogical composition. A significantly greater amount of olivine and smaller amount of melilite are present in the sample of Vig 1623-5 studied by INAA than in the one seen in thin section. Given the spatial segregation of these phases from one another observed in the thin section of Vig 1623-5 and the tiny amount of sample studied by INAA, this discrepancy is easily understandable.

Although we were unable to determine point-count modes in the fine-grained inclusions Vig 1623-14 and Vig 1623-16, we were able to ascertain that the major feldspathoids are nepheline and sodalite in both. Assuming that all $\mathrm{Cl}$ in the INAA determination is present as sodalite and all $\mathrm{Na}$ in excess of the amount in sodalite is present as nepheline, we calculate $30 \%$ sodalite and $15 \%$ nepheline in the 
Table 2. Chemical Compositions of Leoville and Vigarano Inclusions (ppm. unless otherwise noted)

\begin{tabular}{|c|c|c|c|c|c|c|c|c|c|c|c|c|c|c|c|c|c|c|}
\hline Sample & Description & $\begin{array}{c}\text { Mass } \\
(\mu g)\end{array}$ & $\begin{array}{c}\text { Mass } \\
(\mu \mathrm{g}) \\
\end{array}$ & $\begin{array}{l}\mathrm{CaO} \\
(\%) \\
\end{array}$ & $\begin{array}{c}\mathrm{Al}_{2} \mathrm{O}_{3} \\
(\%)\end{array}$ & $\begin{array}{c}\mathrm{MgO} \\
(\%) \\
\end{array}$ & $\mathrm{Na}$ & $\mathrm{K}$ & $\mathrm{Sc}$ & $\begin{array}{c}\mathrm{TiO}_{2} \\
(\%)\end{array}$ & $\mathrm{V}$ & $\mathrm{Cr}$ & $\mathrm{Mn}$ & $\mathrm{Fe}(\%)$ & $\mathrm{Co}$ & $\mathrm{Ni}$ & $\mathrm{Zn}$ & $\mathrm{Ga}$ \\
\hline Leo $3537-2$ & Type B1 CAI & $\begin{array}{r}1049.0 \\
\pm 0.3\end{array}$ & $\begin{array}{l}316.2 \\
\pm 0.6\end{array}$ & $\begin{array}{r}25.2 \\
\pm 0.6\end{array}$ & $\begin{array}{r}34.9 \\
\pm 0.4\end{array}$ & $\begin{array}{r}13.7 \\
\pm 0.3\end{array}$ & $\begin{array}{r}311 \\
\pm 7\end{array}$ & $\begin{array}{r}70.3 \\
\pm 3.6\end{array}$ & $\begin{array}{l}134.9 \\
\pm 0.2\end{array}$ & $\begin{array}{r}1.94 \\
\pm 0.03\end{array}$ & $\begin{array}{r}987 \\
\pm 38\end{array}$ & $\begin{array}{l}408 \\
\pm 1\end{array}$ & $\begin{array}{r}38.2 \\
\pm 1.3\end{array}$ & $\begin{array}{r}0.319 \\
\pm 0.005\end{array}$ & $\begin{array}{r}16.25 \\
\pm 0.05\end{array}$ & $\begin{array}{r}456 \\
\pm 5\end{array}$ & $<18$ & $<0.13$ \\
\hline Vig 477-B & Type Bl CAI & $\begin{array}{r}1162.6 \\
\pm 0.8\end{array}$ & $\begin{array}{l}743.2 \\
\pm 0.5\end{array}$ & $\begin{array}{r}22.9 \\
\pm 0.6\end{array}$ & $\begin{array}{r}37.3 \\
\pm 0.3\end{array}$ & $\begin{array}{r}16.8 \\
\pm 0.4\end{array}$ & $\begin{array}{r}438 \\
\pm 5\end{array}$ & $\begin{array}{r}57.4 \\
\pm 4.1\end{array}$ & $\begin{array}{l}320.7 \\
\pm 0.8\end{array}$ & $\begin{array}{r}2.64 \\
\pm 0.04\end{array}$ & $\begin{array}{l}1210 \\
\pm 50\end{array}$ & $\begin{array}{l}530 \\
\pm 2\end{array}$ & $\begin{array}{r}11.5 \\
\pm 0.5\end{array}$ & $\begin{array}{r}0.411 \\
\pm 0.006\end{array}$ & $\begin{array}{r}40.1 \\
\pm 0.1\end{array}$ & $\begin{array}{r}1038 \\
\pm 6\end{array}$ & $\begin{array}{r}33 \\
\pm 13\end{array}$ & $<1.2$ \\
\hline Vig 477.5 Fl & Fluffy Type A CAI & $\begin{array}{r}49.5 \\
\pm 0.3\end{array}$ & $\begin{array}{r}12.5 \\
\pm 0.8\end{array}$ & $\begin{array}{r}38.2 \\
\pm 1.3\end{array}$ & $\begin{array}{r}35.8 \\
\pm 0.3\end{array}$ & $\begin{array}{r}2.81 \\
\pm 0.44\end{array}$ & $<580$ & $\begin{array}{r}143 \\
\pm 53\end{array}$ & $\begin{array}{l}105.4 \\
\pm 0.1\end{array}$ & $\begin{array}{r}1.11 \\
\pm 0.05\end{array}$ & $\begin{array}{r}254 \\
\pm 9\end{array}$ & $\begin{array}{l}791 \\
\pm 3\end{array}$ & $\begin{array}{r}64.4 \\
\pm 4.2\end{array}$ & $\begin{array}{r}0.874 \\
\pm 0.005\end{array}$ & $\begin{array}{r}12.3 \\
\pm 1.4\end{array}$ & $\begin{array}{l}636 \\
\pm 6\end{array}$ & $\begin{array}{r}34 \\
\pm 10\end{array}$ & $\begin{array}{r}0.31 \\
\pm 0.14\end{array}$ \\
\hline Vig 1623-5 & $\begin{array}{l}\text { Oliv-fass-rich } \\
\text { FUN inclusion }\end{array}$ & $\begin{array}{r}11.9 \\
\pm 0.1\end{array}$ & $\begin{array}{r}8.2 \\
\pm 0.6\end{array}$ & $\begin{array}{r}8.16 \\
\pm 0.83\end{array}$ & $\begin{array}{r}5.56 \\
\pm 0.07\end{array}$ & $\begin{array}{r}43.4 \\
\pm 1.3\end{array}$ & $<350$ & $\begin{array}{r}237 \\
\pm 33\end{array}$ & $\begin{array}{l}358.06 \\
\pm 0.08\end{array}$ & $\begin{array}{r}1.21 \\
\pm 0.08\end{array}$ & $\begin{array}{l}146 \\
\pm 7\end{array}$ & $\begin{array}{l}215 \\
\pm 3\end{array}$ & $\begin{array}{r}28.1 \\
\pm 8.3\end{array}$ & $\begin{array}{r}0.836 \\
\pm 0.012\end{array}$ & $\begin{array}{r}16.9 \\
\pm 0.8\end{array}$ & $\begin{array}{l}9320 \\
\pm 10\end{array}$ & $\begin{array}{r}71 \\
\pm 14\end{array}$ & $<1.1$ \\
\hline Vig 1623-8 & AI & $\begin{array}{l}749.0 \\
\pm 0.9\end{array}$ & $\begin{array}{l}639.7 \\
\pm 0.2\end{array}$ & $\begin{array}{r}27.5 \\
\pm 0.6\end{array}$ & $\begin{array}{r}33.1 \\
\pm 0.2\end{array}$ & $\begin{array}{r}12.4 \\
\pm 0.3\end{array}$ & $\begin{array}{r}585 \\
\pm 5\end{array}$ & $\begin{array}{r}40.9 \\
\pm 3.5\end{array}$ & $\begin{array}{l}161.5 \\
\pm 0.4\end{array}$ & $\begin{array}{r}1.92 \\
\pm 0.03\end{array}$ & $\begin{array}{r}748 \\
\pm 21\end{array}$ & $\begin{array}{l}400 \\
\pm 1\end{array}$ & $\begin{array}{r}16.5 \\
\pm 0.6\end{array}$ & $\begin{array}{r}0.445 \\
\pm 0.006\end{array}$ & $\begin{array}{r}71.6 \\
\pm 0.2\end{array}$ & $\begin{array}{r}2082 \\
\pm 5\end{array}$ & $\begin{array}{r}21.3 \\
\pm 6.4\end{array}$ & $\begin{array}{r}0.32 \\
\pm 0.13\end{array}$ \\
\hline Vig $1623-10$ & Olivine & $\begin{array}{l}268.0 \\
\pm 0.1\end{array}$ & $\begin{array}{r}44.2 \\
\pm 0.2\end{array}$ & $\begin{array}{r}4.43 \\
\pm 0.19\end{array}$ & $\begin{array}{r}5.69 \\
\pm 0.05\end{array}$ & $\begin{array}{r}43.8 \\
\pm 0.5\end{array}$ & $\begin{array}{r}1670 \\
\pm 70\end{array}$ & $\begin{array}{l}1940 \\
\pm 20\end{array}$ & $\begin{array}{r}23.67 \\
\pm 0.02\end{array}$ & $\begin{array}{r}0.250 \\
\pm 0.016\end{array}$ & $\begin{array}{l}170 \\
5 \pm 5\end{array}$ & $\begin{array}{l}695 \\
\pm 1\end{array}$ & $\begin{array}{r}632 \\
\pm 9\end{array}$ & $\begin{array}{r}1.971 \\
\pm 0.006\end{array}$ & $\begin{array}{r}37.9 \\
\pm 0.2\end{array}$ & $\begin{array}{r}6030 \\
\pm 3\end{array}$ & $\begin{array}{r}2340 \\
\pm 20\end{array}$ & $\begin{array}{r}5.07 \\
\pm 0.45\end{array}$ \\
\hline Vig 1623-11 & Type & $\begin{array}{r}96.9 \\
\pm 0.1\end{array}$ & $\begin{array}{r}95.0 \\
\pm 0.3\end{array}$ & $\begin{array}{r}32.9 \\
\pm 1.0\end{array}$ & $\begin{array}{r}30.0 \\
\pm 0.2\end{array}$ & $\begin{array}{r}8.17 \\
\pm 0.42\end{array}$ & $<270$ & $\begin{array}{r}99.7 \\
\pm 6.5\end{array}$ & $\begin{array}{r}78.47 \\
\pm 0.03\end{array}$ & $\begin{array}{r}1.63 \\
\pm 0.05\end{array}$ & $\begin{array}{r}475 \\
\pm 14\end{array}$ & $\begin{array}{r}589 \\
\pm 1\end{array}$ & $\begin{array}{l}227 \\
\pm 8\end{array}$ & $\begin{array}{r}0.646 \\
\pm 0.002\end{array}$ & $\begin{array}{r}32.5 \\
\pm 0.2\end{array}$ & $\begin{array}{r}1093 \\
\pm 2\end{array}$ & $\begin{array}{r}122 \\
\pm 4\end{array}$ & $<0.30$ \\
\hline Vig 1623-13 & $\begin{array}{r}\text { Barre } \\
\text { che }\end{array}$ & $\begin{array}{r}96.7 \\
\pm 0.2\end{array}$ & $\begin{array}{r}68.8 \\
\pm 0.8\end{array}$ & $\begin{array}{r}9.91 \\
\pm 0.58\end{array}$ & $\begin{array}{r}15.2 \\
\pm 0.2\end{array}$ & $\begin{array}{r}34.0 \\
\pm 0.7\end{array}$ & $\begin{array}{l}3660 \\
\pm 80\end{array}$ & $\begin{array}{r}86 \\
\pm 13\end{array}$ & $\begin{array}{r}60.59 \\
\pm 0.03\end{array}$ & $\begin{array}{r}0.392 \\
\pm 0.038\end{array}$ & $\begin{array}{l}2138 \\
8 \pm 6\end{array}$ & $\begin{array}{r}5471 \\
\pm 9\end{array}$ & $\begin{array}{l}223 \\
\pm 6\end{array}$ & $\begin{array}{r}2.883 \\
\pm 0.006\end{array}$ & $\begin{array}{r}6.78 \\
\pm 0.28\end{array}$ & $\begin{array}{r}775 \\
\pm 1.2\end{array}$ & $\begin{array}{r}55.4 \\
\pm 8.1\end{array}$ & $<0.76$ \\
\hline Vig 1623-14 & Fine-grained CAI & $\begin{array}{r}55.5 \\
\pm 0.3\end{array}$ & $\begin{array}{r}51.5 \\
\pm 0.1\end{array}$ & $\begin{array}{r}5.96 \\
\pm 0.49\end{array}$ & $\begin{array}{r}29.4 \\
\pm 0.3\end{array}$ & $\begin{array}{r}6.80 \\
\pm 0.53\end{array}$ & $\begin{array}{l}71400 \\
\pm 200\end{array}$ & $\begin{array}{r}3870 \\
\pm 130\end{array}$ & $\begin{array}{r}32.0 \\
\pm 0.2\end{array}$ & $\begin{array}{r}0.929 \\
\pm 0.067\end{array}$ & $\begin{array}{r}818 \\
7 \pm 25\end{array}$ & $\begin{array}{l}703 \\
\pm 1\end{array}$ & $\begin{array}{l}114 \\
\pm 7\end{array}$ & $\begin{array}{r}2.57 \\
\pm 0.15\end{array}$ & $\begin{array}{r}12.1 \\
\pm 0.3\end{array}$ & $\begin{array}{r}4.9 \\
+1.9\end{array}$ & $\begin{array}{r}1380 \\
\pm 140\end{array}$ & $<9.7$ \\
\hline Vig $1623-16$ & ined CAI & $\begin{array}{r}348.8 \\
\pm 0.1\end{array}$ & $\begin{array}{l}265.8 \\
\pm 0.4\end{array}$ & $\begin{array}{r}9.46 \\
\pm 0.36\end{array}$ & $\begin{array}{r}22.0 \\
\pm 0.3\end{array}$ & $\begin{array}{r}13.0 \\
\pm 0.3\end{array}$ & $\begin{array}{c}31700 \\
\pm 100\end{array}$ & $\begin{array}{l}1700 \\
\pm 70\end{array}$ & $\begin{array}{r}6.14 \\
\pm 0.03\end{array}$ & $\begin{array}{r}4.09 \\
3 \pm 0.03\end{array}$ & $\begin{array}{r}392 \\
\pm 17\end{array}$ & $\begin{array}{r}2387 \\
\pm 5\end{array}$ & $\begin{array}{l}282 \\
\pm 7\end{array}$ & $\begin{array}{r}3.75 \\
\pm 0.06\end{array}$ & $\begin{array}{r}8.64 \\
+0.08\end{array}$ & $\begin{array}{r}206.1 \\
\pm 0.8\end{array}$ & $\begin{array}{r}1320 \\
+80\end{array}$ & $\begin{array}{r}3.4 \\
+1.1\end{array}$ \\
\hline Irradiation ${ }^{1}$ & & $\mathbf{R}$ & $\mathrm{L}$ & $\mathrm{R}$ & $\mathbf{R}$ & $\mathbf{R}$ & $\mathrm{L}$ & L & $\mathrm{L}$ & $\mathrm{R}$ & $\mathbf{R}$ & $\mathbf{L}$ & R & $\mathrm{L}$ & $\mathfrak{L}$ & L & $\mathbf{L}$ & L \\
\hline Nuclide & & & & ${ }^{49} \mathrm{Ca}$ & $28 \wedge 1$ & ${ }^{27} \mathrm{Mg}$ & ${ }^{24} \mathrm{Na}$ & ${ }^{42} \mathrm{~K}$ & ${ }^{46} \mathrm{Sc}$ & ${ }^{51} \mathrm{Ti}$ & $52 \mathrm{~V}$ & ${ }^{51} \mathrm{Cr}$ & ${ }^{56} \mathrm{Mn}$ & ${ }^{59} \mathrm{Fe}$ & ${ }^{60} \mathrm{Co}$ & ${ }^{58} \mathrm{Co}$ & $69 \mathrm{~m} \mathrm{Zn}$ & ${ }^{72} \mathrm{Ga}$ \\
\hline Energy (keV) & & & & 3084 & 1779 & 1014 & 1368 & 1525 & 889 & 320 & 1434 & 320 & 1811 & 1099 & 1332 & 811 & 439 & 630 \\
\hline Half-life & & & & $8.72 \mathrm{~m}$ & $2.25 \mathrm{~m}$ & $9.45 \mathrm{~m}$ & $14.97 \mathrm{~h}$ & $12.36 \mathrm{~h}$ & $83.8 \mathrm{~d}$ & $5.76 \mathrm{~m} 3^{3}$ & $3.76 \mathrm{~m}$ & $27.70 \mathrm{~d}$ & $2.579 \mathrm{~h}$ & $44.51 \mathrm{~d}$ & $5.272 y$ & $70.91 \mathrm{~d}$ & $13.8 \mathrm{~h}$ & $13.95 \mathrm{~h}$ \\
\hline Standard & & & & $\mathrm{CaO}$ & $\mathrm{Al}_{2} \mathrm{O}_{3}$ & $\mathrm{MgO}$ & SP & SP & SP & $\mathrm{TiO}_{2}$ & $\mathrm{BCR}$ & SP & $\mathrm{BCR}$ & SH & SF & $\mathrm{NZ}$ & $\mathrm{NZ}$ & $\mathrm{SP}$ \\
\hline Filomena ${ }^{2}$ & & & $\begin{array}{r}168.2 \\
\pm 0.2\end{array}$ & & & & & & & & & $\begin{array}{r}92.7 \\
\pm 3.8\end{array}$ & & $\begin{array}{r}94.1 \\
\pm 1.2\end{array}$ & $\begin{array}{r}4560 \\
\pm 10\end{array}$ & $\begin{array}{r}57060 \\
\pm 70\end{array}$ & & $\begin{array}{r}59.0 \\
\pm 3.5\end{array}$ \\
\hline Filomena ${ }^{4}$ & & & & & & & & & & & & $\begin{array}{r}68 \\
+9\end{array}$ & & & $\begin{array}{r}4540 \\
+50\end{array}$ & $\begin{array}{l}56500 \\
\pm 600\end{array}$ & & $\begin{array}{r}58.8 \\
\pm 1.1\end{array}$ \\
\hline \multicolumn{4}{|c|}{ Cl Chondrites 5} & 1.30 & 1.64 & 16.4 & 5000 & 558 & 5.82 & 0.0727 & 56.5 & 2660 & 1990 & 19.04 & 502 & 11000 & 312 & 10.0 \\
\hline
\end{tabular}

\footnotetext{
${ }^{1}$ Elements determined in the rabbit irradiation are designated by $\mathrm{R}$; in the long irradiation by $\mathrm{L}$, Quoted uncertainties for each concentration are based on 10 counting statistics errors. ${ }^{2}$ This study. ${ }^{3}$ SYLVESTER et al. (1990b). ${ }^{4}$ Means and standard deviations of replicate analyses from WASSON et al. (1989), except Os and Ru from PERNICKA and WASSON (1987). 5 ANDERS and GREVESSE (1989).
}

sample of Vig 1623-14 which we analyzed and $16 \%$ sodalite and $4 \%$ nepheline in Vig 1623-16.

\section{REFRACTORY ELEMENT CONCENTRATIONS}

\section{Review of Allende Inclusion Compositions and their Interpretation}

Before interpreting refractory element concentrations of the Vigarano and Leoville inclusions analyzed in this study, it is instructive to review what is known about refractory element concentrations of Allende inclusions, and how those concentrations have been interpreted.

Thermodynamic calculations suggest that refractory lithophile $(\mathrm{Ca}$, $\mathrm{Al}, \mathrm{Ti}, \mathrm{REEs}, \mathrm{Hf}, \mathrm{Zr}, \mathrm{Sc}, \mathrm{Th}, \mathrm{Ta}, \mathrm{V}, \mathrm{Sr}, \mathrm{Ba}$ ) and siderophile (W, Re Os, Ir, Mo, Ru) elements would have condensed from a cooling solar nebula gas into host phases such as zirconium oxide, hibonite $\left(\mathrm{CaAl}_{12} \mathrm{O}_{19}\right)$, perovskite $\left(\mathrm{CaTiO}_{3}\right)$, melilite $\left(\mathrm{Ca}_{2} \mathrm{Al}_{2} \mathrm{SiO}_{7}-\mathrm{Ca}_{2} \mathrm{MgSi}_{2} \mathrm{O}_{7}\right)$, and metal alloys at high temperatures (GROSSMAN, 1972, 1973; BOYNTON, 1975; PALME and WLOTZKA, 1976; HINTON et al., 1988; SYLVESTER et al., 1990b). More volatile elements (Ni, Co, Mg, Fe, $\mathrm{Si}, \mathrm{Cr}, \mathrm{Au}, \mathrm{Mn}, \mathrm{As}, \mathrm{K}, \mathrm{Na}, \mathrm{Ga}, \mathrm{Sb}, \mathrm{Cl}, \mathrm{Se}, \mathrm{Cs}, \mathrm{Zn}, \mathrm{Br}$ ) would have condensed from the same gas into host phases such as metallic nickeliron, troilite, olivine, pyroxene, and feldspar at lower temperatures. Allende refractory inclusions are thought to have been assembled from high-temperature, refractory element-rich condensate phases or evaporation residues that were removed from the nebular gas before condensation of lower temperature, volatile element-rich phases. This is because such inclusions are enriched in refractory elements by factors of ten or more and depleted in most volatile elements by at least $10 \%$, compared to element concentrations in $\mathrm{Cl}$ chondrites, which are probably representative of the total condensable matter in the solar system (GROSSMAN and GANAPATHY, 1976a,b). Original high-temperature condensates or residues still exist as the constituent minerals of inclusions that have never been melted, such as in the melilite-rich fluffy Type As of Allende (MACPHERSON and GrossMAN, 1984), but, in melted inclusions such as melilite-rich compact Type As and fassaite-rich Type Bs of Allende, original condensate phases have been largely consumed during fusion and the minerals now present crystallized from melts (MACPHERSON and GROSSMAN, 1981). Refractory elements in melted inclusions have been redistributed among the crystallizing minerals, according to crystal-melt partition coefficients for each element in each mineral.

Most coarse-grained, Type A and B Allende inclusions have rare earth element (REE) concentrations enriched to $\sim 18$ times their levels in $\mathrm{Cl}$ chondrites and either Group I or modified Group I chondrite-normalized REE patterns (GROSSMAN et al., 1977; MASON and MARTIN, 1977). Group I RFE patterns are those in which no REE is fractionated from any other, except Eu, by more than $20 \%$ relative to $\mathrm{Cl}$ chondrites. Precursors of inclusions with such patterns were probably isolated from the nebular gas at temperatures low enough 
Table 2 (continued)

\begin{tabular}{|c|c|c|c|c|c|c|c|c|c|c|c|c|c|c|c|c|c|c|}
\hline Sample & As & $\mathrm{Se}$ & $\mathrm{Br}$ & $\mathrm{Cl}$ & Sr & $\mathrm{Zr}$ & $\begin{array}{c}\mathrm{Sb} \\
(\mathrm{ppb})\end{array}$ & $\begin{array}{c}\mathrm{Cs} \\
(\mathrm{ppb})\end{array}$ & Ba & $\mathrm{La}$ & $\mathrm{Ce}$ & $\mathrm{Sm}$ & $\mathrm{Eu}$ & $\mathrm{Tb}$ & Dy & Ho & $\operatorname{Tm}$ & $\mathrm{Yb}$ \\
\hline Leo $3537-2$ & $<0.072$ & $<037$ & $<0.11$ & $<47$ & $\begin{array}{r}144 \\
\pm 12\end{array}$ & $\begin{array}{r}69.7 \\
\pm 8.8\end{array}$ & $<14$ & $\begin{array}{r}92 \\
\pm 13\end{array}$ & $<52$ & $\begin{array}{r}3.70 \\
\pm 0.04\end{array}$ & $\begin{array}{r}9.41 \\
\pm 0.24\end{array}$ & $\begin{array}{r}2.667 \\
\pm 0.008\end{array}$ & $\begin{array}{r}0.978 \\
\pm 0.009\end{array}$ & $\begin{array}{r}0.691 \\
\pm 0.018\end{array}$ & $\begin{array}{r}4.93 \\
\pm 0.18\end{array}$ & $\begin{array}{r}1.04 \\
\pm 0.05\end{array}$ & $\begin{array}{r}0.499 \\
\pm 0.060\end{array}$ & $\begin{array}{r}2.68 \\
+0.05\end{array}$ \\
\hline Vig 477-B & $<0.11$ & $<0.67$ & $\begin{array}{r}0.347 \\
\pm 0.058\end{array}$ & $<52$ & $\begin{array}{r}131 \\
\pm 14\end{array}$ & $\begin{array}{r}229 \\
\pm 17\end{array}$ & $<7.4$ & $<34$ & $<72$ & $\begin{array}{r}5.76 \\
\pm 0.04\end{array}$ & $\begin{array}{r}11.7 \\
\pm 0.4\end{array}$ & $\begin{array}{r}3.73 \\
\pm 0.01\end{array}$ & $\begin{array}{r}0.790 \\
\pm 0.014\end{array}$ & $\begin{array}{r}1.89 \\
\pm 0.03\end{array}$ & $\begin{array}{r}15.3 \\
\pm 0.5\end{array}$ & $\begin{array}{r}3.16 \\
+0.09\end{array}$ & $\begin{array}{r}2.39 \\
\pm 0.05\end{array}$ & $\begin{array}{r}4.80 \\
\pm 0.06\end{array}$ \\
\hline Vig $477-5$ & $\begin{array}{r}0.440 \\
\pm 0.054\end{array}$ & $\begin{array}{r}0.47 \\
\pm 0.11\end{array}$ & $\begin{array}{r}5.9 \\
\pm 2.4\end{array}$ & $\begin{array}{r}850 \\
\pm 210\end{array}$ & $\begin{array}{r}205 \\
\pm 10\end{array}$ & $\begin{array}{r}35.2 \\
\pm 8.3\end{array}$ & $<240$ & $<27$ & $<44$ & $\begin{array}{r}5.38 \\
\pm 0.05\end{array}$ & $\begin{array}{r}11.0 \\
\pm 0.2\end{array}$ & $\begin{array}{r}3.214 \\
\pm 0.008\end{array}$ & $\begin{array}{r}1.35 \\
\pm 0.03\end{array}$ & $\begin{array}{r}0.937 \\
\pm 0.012\end{array}$ & $\begin{array}{r}6.09 \\
\pm 0.37\end{array}$ & $\begin{array}{r}1.59 \\
\pm 0.06\end{array}$ & $\begin{array}{r}0.634 \\
\pm 0.018\end{array}$ & $\begin{array}{r}2.33 \\
\pm 0.03\end{array}$ \\
\hline Vig 1623-5 & $\begin{array}{r}2.40 \\
\pm 0.10\end{array}$ & $<1.2$ & $\begin{array}{r}293 \\
\pm 1.7\end{array}$ & $<1200$ & $<25$ & $\begin{array}{r}78 \\
\pm 15\end{array}$ & $<390$ & $<100$ & $<110$ & $\begin{array}{r}2.03 \\
\pm 0.05\end{array}$ & $\begin{array}{r}6.38 \\
\pm 0.23\end{array}$ & $\begin{array}{r}2.01 \\
\pm 0.01\end{array}$ & $<0.084$ & $\begin{array}{r}0.554 \\
\pm 0.022\end{array}$ & $\begin{array}{r}2.38 \\
\pm 0.72\end{array}$ & $\begin{array}{r}0.890 \\
\pm 0.066\end{array}$ & $\begin{array}{r}0.336 \\
\pm 0.046\end{array}$ & $\begin{array}{r}2.81 \\
\pm 0.05\end{array}$ \\
\hline Vig 1623-8 & $<0.074$ & $<034$ & $<0.12$ & $<14$ & $\begin{array}{r}135 \\
\pm 8\end{array}$ & $\begin{array}{r}67.0 \\
\pm 8.7\end{array}$ & $<11$ & $<\boldsymbol{w}$ & $<41$ & $\begin{array}{r}3.91 \\
\pm 0.03\end{array}$ & $\begin{array}{r}8.32 \\
\pm 0.19\end{array}$ & $\begin{array}{r}2.297 \\
\pm 0.007\end{array}$ & $\begin{array}{r}1.12 \\
\pm 0.01\end{array}$ & $\begin{array}{r}0.726 \\
\pm 0.015\end{array}$ & $\begin{array}{r}5.18 \\
\pm 0.20\end{array}$ & $\begin{array}{r}1.15 \\
\pm 0.04\end{array}$ & $\begin{array}{r}0.527 \\
\pm 0.053\end{array}$ & $\begin{array}{r}3.34 \\
\pm 0.03\end{array}$ \\
\hline Vig $1623-10$ & $\begin{array}{r}0.178 \\
\pm 0.020\end{array}$ & $\begin{array}{r}20.00 \\
\pm 0.06\end{array}$ & $\begin{array}{r}22.2 \\
\pm 0.7\end{array}$ & $\begin{array}{r}329 \\
\pm 60\end{array}$ & $<4.1$ & $\begin{array}{r}22.8 \\
\pm 2.9\end{array}$ & $\begin{array}{r}714 \\
\pm 34\end{array}$ & $\begin{array}{l}465 \\
\pm 8\end{array}$ & $<19$ & $\begin{array}{r}1.15 \\
\pm 0.01\end{array}$ & $\begin{array}{r}2.50 \\
\pm 0.04\end{array}$ & $\begin{array}{r}0.507 \\
\pm 0.002\end{array}$ & $\begin{array}{r}0.249 \\
\pm 0.008\end{array}$ & $\begin{array}{r}0.139 \\
\pm 0.005\end{array}$ & $\begin{array}{r}0.93 \\
\pm 0.16\end{array}$ & $\begin{array}{r}0.204 \\
\pm 0.020\end{array}$ & $\begin{array}{r}0.0967 \\
\pm 0.0064\end{array}$ & $\begin{array}{r}0.632 \\
\pm 0.009\end{array}$ \\
\hline Vig 1623-11 & $\begin{array}{r}0.154 \\
\pm 0.016\end{array}$ & $<0.072$ & $\begin{array}{r}6.08 \\
\pm 0.33\end{array}$ & $\begin{array}{r}560 \\
\pm 180\end{array}$ & $\begin{array}{l}180 \\
\pm 5\end{array}$ & $\begin{array}{r}43.4 \\
\pm 3.8\end{array}$ & $\begin{array}{r}357 \\
\pm 20\end{array}$ & $<21$ & $<17$ & $\begin{array}{r}3.21 \\
\pm 0.02\end{array}$ & $\begin{array}{r}8.09 \\
\pm 0.06\end{array}$ & $\begin{array}{r}1.828 \\
\pm 0.002\end{array}$ & $\begin{array}{r}1.33 \\
\pm 0.01\end{array}$ & $\begin{array}{r}0.638 \\
\pm 0.021\end{array}$ & $\begin{array}{r}4.35 \\
\pm 0.61\end{array}$ & $\begin{array}{r}0.975 \\
\pm 0.036\end{array}$ & $\begin{array}{r}0.446 \\
\pm 0.018\end{array}$ & $\begin{array}{r}3.05 \\
\pm 0.01\end{array}$ \\
\hline$V_{i g} 1623-13$ & $\begin{array}{r}0.26 \\
\pm 0.10\end{array}$ & $<0.14$ & $\begin{array}{r}8.77 \\
\pm 0.39\end{array}$ & $\begin{array}{r}2100 \\
\pm 190\end{array}$ & $\begin{array}{r}65.4 \\
\pm 3.3\end{array}$ & $\begin{array}{r}20.6 \\
\pm 3.0\end{array}$ & $<46$ & $\begin{array}{r}11.4 \\
\pm 6.6\end{array}$ & $<15$ & $\begin{array}{r}1.27 \\
\pm 0.02\end{array}$ & $\begin{array}{r}2.93 \\
\pm 0.05\end{array}$ & $\begin{array}{r}0.874 \\
\pm 0.002\end{array}$ & $\begin{array}{r}0.374 \\
\pm 0.007\end{array}$ & $\begin{array}{r}0.259 \\
\pm 0.008\end{array}$ & $\begin{array}{r}1.75 \\
\pm 0.43\end{array}$ & $\begin{array}{r}0.431 \\
\pm 0.019\end{array}$ & $\begin{array}{r}0.179 \\
+0.010\end{array}$ & $\begin{array}{r}1.209 \\
\pm 0.015\end{array}$ \\
\hline Vig 1623-14 & $<056$ & $<0.061$ & $\begin{array}{r}55.6 \\
\pm 1.8\end{array}$ & $\begin{array}{l}16600 \\
\pm 500\end{array}$ & $\begin{array}{r}775 \\
\pm 2.6\end{array}$ & $<8.0$ & $<24$ & $\begin{array}{r}51.9 \\
\pm 5.0\end{array}$ & $\begin{array}{r}4.8 \\
+2.0\end{array}$ & $\begin{array}{r}9.63 \\
\pm 0.17\end{array}$ & $\begin{array}{r}25.14 \\
\pm 0.05\end{array}$ & $\begin{array}{r}5.66 \\
\pm 0.04\end{array}$ & $\begin{array}{r}0.285 \\
\pm 0.007\end{array}$ & $\begin{array}{r}0.860 \\
\pm 0.006\end{array}$ & $\begin{array}{r}3.92 \\
\pm 0.66\end{array}$ & $\begin{array}{r}0.55 \\
\pm 0.19\end{array}$ & $\begin{array}{r}0.988 \\
\pm 0.003\end{array}$ & $\begin{array}{r}1.093 \\
\pm 0.012\end{array}$ \\
\hline Vig 1623-16 & $<0.20$ & $<0.030$ & $\begin{array}{r}36.3 \\
\pm 1.1\end{array}$ & $\begin{array}{r}8530 \\
\pm 190\end{array}$ & $\begin{array}{r}93.4 \\
\pm 1.2\end{array}$ & $<15$ & $<4.6$ & $\begin{array}{r}89.3 \\
\pm 2.4\end{array}$ & $\begin{array}{r}34.4 \\
\pm 1.7\end{array}$ & $\begin{array}{r}1.36 \\
\pm 0.03\end{array}$ & $\begin{array}{r}6.01 \\
\pm 0.02\end{array}$ & $\begin{array}{r}1.217 \\
\pm 0.008\end{array}$ & $\begin{array}{r}0.389 \\
\pm 0.003\end{array}$ & $\begin{array}{r}0.069 \\
+0.001\end{array}$ & $<0.42$ & $<0.10$ & $\begin{array}{r}0.572 \\
\pm 0.003\end{array}$ & $\begin{array}{r}1.298 \\
\pm 0.004\end{array}$ \\
\hline Irradiation ${ }^{1}$ & $\mathrm{~L}$ & $\mathrm{~L}$ & $\mathbf{L}$ & $\mathbf{R}$ & L & $\mathbf{L}$ & L & $\mathbf{L}$ & $\mathrm{L}$ & $\mathrm{L}$ & L & $\mathbf{L}$ & $\mathrm{L}$ & L & $\mathbf{R}$ & $\mathrm{L}$ & $\mathbf{L}$ & $\mathrm{L}$ \\
\hline Nuclide & ${ }^{76} \mathrm{As}$ & ${ }^{75} \mathrm{Se}$ & ${ }^{82} \mathrm{Br}$ & ${ }^{38} \mathrm{Cl}$ & ${ }^{85} \mathrm{Sr}$ & ${ }^{95} \mathbf{Z}_{\mathbf{r}}$ & ${ }^{124} \mathrm{Sb}$ & ${ }^{134} \mathrm{Cs}$ & $131_{\mathrm{Ba}}$ & ${ }^{140} \mathrm{La}$ & ${ }^{141} \mathrm{Ce}$ & ${ }^{153} \mathrm{Sm}$ & ${ }^{152} \mathrm{Eu}$ & ${ }^{160} \mathrm{~Tb}$ & ${ }^{165} \mathrm{Dy}$ & ${ }^{166} \mathrm{Ho}$ & ${ }^{170} \mathrm{Tm}$ & $175 \mathrm{Yb}$ \\
\hline Energy $(\mathrm{keV})$ & 559 & 265 & 776 & 1643 & 514 & 757 & 1691 & 796 & 373 & 1596 & 145 & 103 & 1408 & 8791178 & 95 & 81 & 84 & 396 \\
\hline Half-life & $26.3 \mathrm{~h}$ & $119.78 \mathrm{~d}$ & $35.30 \mathrm{~h}$ & $37.2 \mathrm{~m}$ & $64.8 \mathrm{~d}$ & $64.03 d$ & $60.20 \mathrm{~d}$ & $2.065 \mathrm{y}$ & $11.8 \mathrm{~d}$ & $40.28 \mathrm{~h}$ & $32.5 \mathrm{~d}$ & $46.7 \mathrm{~h}$ & $13.4 \mathrm{y}$ & $72.4 \mathrm{~d}$ & $2.33 \mathrm{~h}$ & $26.8 \mathrm{~h}$ & $129 \mathrm{~d}$ & $4.19 \mathrm{~d}$ \\
\hline Standard & $\mathrm{SP}$ & $\mathrm{NZ}$ & $\mathbf{S P}$ & $\mathrm{KCl}$ & BCR & SP & SP & sp & SP & REEA & REEB & REEB & REEA & REEA & $\mathbf{S P}$ & REEA & REEB & REEA \\
\hline Filomena ${ }^{2}$ & $\begin{array}{r}5.07 \\
\pm 0.12\end{array}$ & & & & & & & & & & & & & & & & & \\
\hline Filomena $^{3}$ & $\begin{array}{r}4.96 \\
\pm 0.33\end{array}$ & & & & & & & & & & & & & & & & & \\
\hline Filomena ${ }^{4}$ & $\begin{array}{r}4.73 \\
\pm 0.09\end{array}$ & & & & & & & & & & & & & & & & & \\
\hline $\mathrm{Cl}$ Chondrites 5 & 1.86 & 18.6 & 3.57 & 704 & 7.80 & 3.94 & 142 & 187 & 2.34 & 0.2347 & 0.6032 & 0.1471 & 0.056 & 0.0363 & 0.2427 & 0.0556 & 0.0242 & 0.1625 \\
\hline
\end{tabular}

that each REE was fully condensed into them. Modified Group I REE patterns are those in which $\mathrm{Cl}$ chondrite-normalized enrichment factors vary progressively with atomic number such that fractionations among REEs exceed $20 \%$. Inclusions with such patterns probably sampled high-temperature condensates or evaporation residues with a strong crystal-chemical preference for either light REEs (LREEs) or heavy REEs (HREEs) in relative proportions that differed from those generally present in the nebula. A high-temperature condensate phase that prefers LREEs is hibonite; one that prefers HREEs is zirconium oxide (HINTON et al., 1988). Group I and modified Group I REE patterns of some Allende inclusions have positive and negative Eu anomalies, which are presumably the result of nonrepresentative sampling of melilite relative to other phases by the inclusions in the nebula. Melilite has a strong crystal-chemical preference for divalent Eu relative to trivalent REEs (KUEHNER et al., 1989), and these are the respective valence states expected under the reducing conditions of a high-temperature gas of solar composition.

In some Allende inclusions with Group I or modified Group I REE patterns, the ratio of any one of $\mathrm{Zr}, \mathrm{Th}, \mathrm{Hf}, \mathrm{Sc}, \mathrm{Ta}, \mathrm{V}, \mathrm{Sr}$, or $\mathrm{Ba}$ to a REE of similar volatility may differ from the ratio of the same elements in $\mathrm{Cl}$ chondrites by more than 20\%. BOYNTON (1975) showed that Lu and Er are the two most refractory REEs, Eu and $\mathrm{Yb}$ the two most volatile REEs, and that $\mathrm{La}$ and $\mathrm{Sm}$ are of intermediate volatility. The condensation calculations of DAVIS et al. (1982) suggest that, in a gas of solar composition and assuming ideal solid solution in Ca-bearing minerals, $\mathrm{Zr}$, Hf, and $\mathrm{Sc}$ are about as refractory as $\mathrm{Lu}$ and $\mathrm{Er}, \mathrm{Ta}$ is about as refractory as $\mathrm{La}$; and the volatilities of V, St, and Ba are equal to or slightly greater than that of $\mathrm{Yb}$. Although the same calculations predict Th to be almost as refractory as $\mathrm{Lu}$, this does not seem to have been the case during formation of Allende inclusion precursors. In Allende Group II inclusions (discussed later), REEs are fractionated from one another as a function of volatility and $T h$ seems to have behaved as a much less refractory element than $\mathrm{Lu}$, as pointed out by BOYNTON (1978), having instead a volatility comparable to that of $\mathrm{La}$ and $\mathrm{Sm}$. In nine Group I and modified Group I inclusions analyzed by Grossman and coworkers (GROSSMAN and GANAPATHY, 1976a; GROSSMAN et al., 1977, and unpubl. data), $\mathrm{Hf} / \mathrm{Lu}$ ranges from $0.40 \pm 0.07$ to 1.02 $\pm 0.04 \times \mathrm{Cl}$ (i.e., multiplied by the same ratio in $\mathrm{Cl}$ chondrites) and $\mathrm{Sc} / \mathrm{Lu}$ from $0.66 \pm 0.04$ to $2.7 \pm 0.2 \times \mathrm{Cl}$. In seven of the nine inclusions, $\mathrm{V} / \mathrm{Yb}$ ranges from $0.22 \pm 0.01$ to $0.99 \pm 0.04 \times \mathrm{Cl}$, and, in five of them, $\mathrm{Zr} / \mathrm{Lu}$ ranges from $0.54 \pm 0.07$ to $0.85 \pm 0.03 \times \mathrm{Cl}$, $\mathrm{Ta} / \mathrm{La}$ from $0.74 \pm 0.16$ to $1.41 \pm 0.09 \times \mathrm{Cl}, \mathrm{Sr} / \mathrm{Yb}$ from 0.63 \pm 0.05 to $3.0 \pm 0.5 \times \mathrm{Cl}$, and $\mathrm{Ba} / \mathrm{Yb}$ from $0.65 \pm 0.06$ to $\mathrm{I} .1 \pm 0.2$ $\times \mathrm{Cl}$. In fourteen Group I and modified Group I inclusions analyzed by Mason and coworkers (MASON and MARTIN, 1977; MASON and TAYLOR, 1982), $\mathrm{Zr} / \mathrm{Er}$ ranges from 0.59 to $1.1 \times \mathrm{Cl}$. In thirteen of these, $\mathrm{Hf} / \mathrm{Er}$ is $0.55-1.1 \times \mathrm{Cl}$, and in twelve of them, Th/La is $0.70-$ $1.1 \times \mathrm{Cl}$. Nine of the inclusions have $\mathrm{Sr} / \mathrm{Yb}$ ranging from 0.65 to $1.8 \times \mathrm{Cl}$ and $\mathrm{Ba} / \mathrm{Yb}$ from 0.67 to $2.8 \times \mathrm{Cl}$.

The cause of these relatively large fractionations is probably related to the fact that the refractory lithophiles do not form ideal solid solutions in all of the available Ca-bearing host phases (BOYNTON, 1975; Hinton et al., 1988). Calcium sites in different minerals are nonequivalent, resulting in crystal-chemical preferences for one phase relative to another during condensation or evaporation of some 
Table 2 (continued)

\begin{tabular}{|c|c|c|c|c|c|c|c|c|c|c|c|c|}
\hline Sample & Lu & $\mathrm{Hf}$ & $\begin{array}{c}\mathrm{Ta} \\
(\mathrm{ppb})\end{array}$ & W & $\begin{array}{c}\operatorname{Re} \\
(\mathrm{ppb})\end{array}$ & Os & Ir & Mo & $\mathrm{Ru}$ & $\begin{array}{c}\mathrm{Au} \\
(\mathrm{ppb})\end{array}$ & $\begin{array}{c}\text { Th } \\
(\mathrm{ppb})\end{array}$ & $\begin{array}{c}\mathrm{U} \\
(\mathrm{ppb})\end{array}$ \\
\hline Leo $3537-2$ & $\begin{array}{r}0.407 \\
\pm 0.017\end{array}$ & $\begin{array}{r}2.81 \\
\pm 0.03\end{array}$ & $\begin{array}{l}208 \\
\pm 8\end{array}$ & $\begin{array}{r}1.03 \\
\pm 0.05\end{array}$ & $\begin{array}{r}541 \\
\pm 46\end{array}$ & $\begin{array}{r}8.43 \\
\pm 0.13\end{array}$ & $\begin{array}{r}7.31 \\
\pm 0.01\end{array}$ & $\begin{array}{r}5.72 \\
\pm 0.51\end{array}$ & $\begin{array}{r}11.2 \\
\pm 0.2\end{array}$ & $<43$ & $\begin{array}{r}295 \\
\pm 28\end{array}$ & $<100$ \\
\hline Vig 477-B & $\begin{array}{r}1.29 \\
\pm 0.02\end{array}$ & $\begin{array}{r}7.27 \\
\pm 0.07\end{array}$ & $\begin{array}{r}404 \\
\pm 15\end{array}$ & $\begin{array}{r}0.451 \\
\pm 0.051\end{array}$ & $\begin{array}{r}483 \\
\pm 35\end{array}$ & $\begin{array}{r}6.30 \\
\pm 0.13\end{array}$ & $\begin{array}{r}6.25 \\
\pm 0.01\end{array}$ & $\begin{array}{r}7.01 \\
\pm 0.61\end{array}$ & $\begin{array}{r}7.57 \\
\pm 0.25\end{array}$ & $\begin{array}{r}4.7 \\
\pm 2.2\end{array}$ & $\begin{array}{r}666 \\
\pm 35\end{array}$ & $<130$ \\
\hline Vig 477-5 & $\begin{array}{r}0.609 \\
\pm 0.024\end{array}$ & $\begin{array}{r}1.75 \\
\pm 0.14\end{array}$ & $\begin{array}{r}191 \\
\pm 22\end{array}$ & $<2.1$ & $\begin{array}{r}546 \\
\pm 28\end{array}$ & $\begin{array}{r}7.31 \\
\pm 0.08\end{array}$ & $\begin{array}{r}6.111 \\
\pm 0.005\end{array}$ & $\begin{array}{r}26.5 \\
\pm 0.4\end{array}$ & $\begin{array}{r}9.58 \\
+0.11\end{array}$ & $\begin{array}{r}45.9 \\
\pm 2.1\end{array}$ & $\begin{array}{r}238 \\
\pm 27\end{array}$ & $<170$ \\
\hline Vig 1623-5 & $\begin{array}{r}0.349 \\
\pm 0.015\end{array}$ & $\begin{array}{r}4.40 \\
\pm 0.23\end{array}$ & $\begin{array}{r}200 \\
\pm 71\end{array}$ & $<25$ & $\begin{array}{r}83.1 \\
\pm 5.9\end{array}$ & $\begin{array}{r}1.40 \\
\pm 0.17\end{array}$ & $\begin{array}{r}1.618 \\
+0.003\end{array}$ & $\begin{array}{r}1.97 \\
\pm 0.35\end{array}$ & $\begin{array}{r}2.50 \\
\pm 0.16\end{array}$ & $\begin{array}{l}177 \\
\pm 3\end{array}$ & $\begin{array}{r}200 \\
\pm 34\end{array}$ & $<140$ \\
\hline Vig 1623-8 & $\begin{array}{r}0.527 \\
\pm 0.019\end{array}$ & $\begin{array}{r}2.41 \\
\pm 0.02\end{array}$ & $\begin{array}{r}206 \\
\pm 11\end{array}$ & $\begin{array}{r}1,41 \\
\pm 0.06\end{array}$ & $\begin{array}{r}743 \\
\pm 30\end{array}$ & $\begin{array}{r}11.7 \\
\pm 0.1\end{array}$ & $\begin{array}{r}10.48 \\
\pm 0.02\end{array}$ & $\begin{array}{r}14.5 \\
\pm 0.5\end{array}$ & $\begin{array}{r}18.0 \\
\pm 0.1\end{array}$ & $\begin{array}{r}3.98 \\
\pm 0.90\end{array}$ & $\begin{array}{r}478 \\
\pm 11\end{array}$ & $<110$ \\
\hline Vig $1623-10$ & $\begin{array}{r}0.0777 \\
\pm 0.0022\end{array}$ & $\begin{array}{r}0.839 \\
+0.039\end{array}$ & $\begin{array}{r}38.5 \\
\pm 5.3\end{array}$ & $<14$ & $\begin{array}{r}79.1 \\
\pm 7.9\end{array}$ & $\begin{array}{r}1.33 \\
\pm 0.04\end{array}$ & $\begin{array}{r}2.224 \\
\pm 0.002\end{array}$ & $\begin{array}{r}3.66 \\
\pm 0.09\end{array}$ & $\begin{array}{r}2.10 \\
\pm 0.03\end{array}$ & $\begin{array}{r}90.7 \\
\pm 0.6\end{array}$ & $\begin{array}{r}55.1 \\
\pm 6.0\end{array}$ & $<29$ \\
\hline Vig 1623-11 & $\begin{array}{r}0.466 \\
\pm 0.004\end{array}$ & $\begin{array}{r}1.69 \\
\pm 0.02\end{array}$ & $\begin{array}{l}159 \\
\pm 9\end{array}$ & $\begin{array}{r}0.70 \\
\pm 0.18\end{array}$ & $\begin{array}{r}236 \\
\pm 7\end{array}$ & $\begin{array}{r}3.06 \\
\pm 0.04\end{array}$ & $\begin{array}{r}2.802 \\
\pm 0.002\end{array}$ & $\begin{array}{r}10.4 \\
\pm 0.1\end{array}$ & $\begin{array}{r}4.67 \\
+0.06\end{array}$ & $\begin{array}{r}7.4 \\
\pm 1.1\end{array}$ & $\begin{array}{r}150 \\
\pm 24\end{array}$ & $\begin{array}{r}129 \\
\pm 28\end{array}$ \\
\hline Vig 1623-13 & $\begin{array}{r}0.161 \\
\pm 0.003\end{array}$ & $\begin{array}{r}0.912 \\
\pm 0.028\end{array}$ & $\begin{array}{r}742 \\
\pm 5.9\end{array}$ & $<0.42<$ & $<100$ & $<0.011$ & $\begin{array}{r}0.00127 \\
\pm 0.00019\end{array}$ & $<039$ & $<0.18$ & $<2,4$ & $\begin{array}{r}130 \\
\pm 16\end{array}$ & $<52$ \\
\hline Vig 1623-14 & $<0.19$ & $<0.019$ & $\begin{array}{r}498 \\
\pm 10\end{array}$ & $<13$ & $<190$ & $<0.012$ & $\begin{array}{r}0.00595 \\
\pm 0.00024\end{array}$ & $<7.4$ & $<0.043$ & $<30$ & $\begin{array}{r}879 \\
\pm 8\end{array}$ & $<770$ \\
\hline Vig $1623-16$ & $\begin{array}{r}0.080 \\
\pm 0.014\end{array}$ & $<0.0045$ & $\begin{array}{l}936 \\
\pm 9\end{array}$ & $<0.63$ & $<37$ & $<0.0042$ & $\begin{array}{r}0.00879 \\
+0.00008\end{array}$ & $<13$ & $<0.013$ & $<5.9$ & $\begin{array}{l}160 \\
\pm 3\end{array}$ & $<160$ \\
\hline
\end{tabular}

\begin{tabular}{|c|c|c|c|c|c|c|c|c|c|c|c|c|}
\hline Irıduiation 1 & $\mathrm{~L}$ & $\mathrm{~L}$ & $\mathrm{~L}$ & $\mathrm{~L}$ & $\mathbf{L}$ & L & $\mathrm{L}$ & L & $\mathrm{L}$ & $\mathrm{L}$ & $\mathrm{L}$ & $\mathbf{L}$ \\
\hline Nuclide & $177_{\mathrm{Lu}}$ & ${ }^{181} \mathrm{Hf}$ & ${ }^{182} \mathrm{Ta}$ & ${ }^{187} \mathrm{~W}$ & ${ }^{186} \mathrm{Re}$ & ${ }^{191} \mathrm{Os}$ & ${ }^{192} \mathrm{Ir}$ & ${ }^{99} \mathrm{Mo}$ & ${ }^{103} \mathrm{Ru}$ & ${ }^{198} \mathrm{Au}$ & ${ }^{233} \mathrm{~Pa}$ & ${ }^{239} \mathrm{~Np}$ \\
\hline Energy (keV) & 208 & 482 & 1221 & 686 & 137 & 129 & 468 & 140 & 497 & 412 & 312 & 278 \\
\hline Half-life & $6.71 \mathrm{~d}$ & $42.4 d$ & $114.5 \mathrm{~d}$ & $23.9 \mathrm{~h}$ & $90.7 \mathrm{~h}$ & $15.4 d$ & $73.83 \mathrm{~d}$ & $65.94 \mathrm{~h}$ & $39.24 \mathrm{~d}$ & $2.6935 d$ & $27.0 \mathrm{~d}$ & $56.4 \mathrm{~h}$ \\
\hline Standard & REEB & SP & SP & $\mathbf{w}$ & $A R$ & AR & AR & Mo & $A R$ & AR & SP & SP \\
\hline Filomena ${ }^{2}$ & & & & $\begin{array}{r}2.57 \\
\pm 0.14\end{array}$ & $\begin{array}{r}264 \\
\pm 40\end{array}$ & $\begin{array}{r}0.94 \\
\pm 0.20\end{array}$ & $\begin{array}{r}3.31 \\
\pm 0.01\end{array}$ & & $\begin{array}{r}17.9 \\
\pm 1.2\end{array}$ & $\begin{array}{l}613 \\
\pm 6\end{array}$ & & \\
\hline Filomena ${ }^{3}$ & & & & & $\begin{array}{r}230 \\
\pm 26\end{array}$ & $\begin{array}{r}1.06 \\
\pm 0.07\end{array}$ & $\begin{array}{r}3.44 \\
\pm 0.03\end{array}$ & & $\begin{array}{r}17.6 \\
\pm 0.4\end{array}$ & $\begin{array}{r}609 \\
\pm 4\end{array}$ & & \\
\hline Filomena ${ }^{4}$ & & & & $\begin{array}{r}2.562 \\
+0.013\end{array}$ & $\begin{array}{r}234 \\
\pm 14\end{array}$ & $\begin{array}{r}1.14 \\
\pm 0.13\end{array}$ & $\begin{array}{r}3.37 \\
\pm 0.05\end{array}$ & & $\begin{array}{r}19.3 \\
\pm 1.2\end{array}$ & $\begin{array}{r}612 \\
\pm 8\end{array}$ & & \\
\hline Cl Chondrites & 0.0243 & 0.104 & 14.2 & 0.0926 & 36.5 & 0.486 & 0.481 & 0.928 & 0.712 & $2 \quad 140$ & 29.4 & 8.1 \\
\hline
\end{tabular}

elements. If, during accretion of inclusion precursors, phases containing distinct ratios of refractory lithophiles were sampled in different proportions from those that existed in the nebula, the fractionations

Table 3. Mean and Standard Deviation of the Mean of the Masses $(\mu \mathrm{g})$ of Elements in Empty Supersilica Tubes

Mao et al. $(1991)^{1} \quad$ This Study ${ }^{2}$

\begin{tabular}{lcc} 
& Mao el al. (1991) & This Sudy \\
\hline $\mathrm{Na}$ & $2.24 \pm 0.74 \times 10^{-2}$ & $7.89 \pm 1.31 \times 10^{-3}$ \\
$\mathrm{Sc}$ & $7.12 \pm 1.53 \times 10^{-6}$ & $<6.1 \pm 1.2 \times 10^{-7}$ \\
$\mathrm{Cr}$ & $5.35 \pm 2.07 \times 10^{-4}$ & $9.32 \pm 1.28 \times 10^{-5}$ \\
$\mathrm{Fe}$ & $4.94 \pm 2.20 \times 10^{-3}$ & $<7.4 \pm 1.7 \times 10^{-3}$ \\
$\mathrm{Co}$ & $3.74 \pm 2.33 \times 10^{-4}$ & $2.28 \pm 0.78 \times 10^{-5}$ \\
$\mathrm{Ni}$ & $2.48 \pm 1.11 \times 10^{-3}$ & $4.16 \pm 1.30 \times 10^{-5}$ \\
$\mathrm{Zn}$ & $2.03 \pm 0.47 \times 10^{-4}$ & $<2.0 \pm 0.4 \times 10^{-4}$ \\
$\mathrm{Br}$ & $2.57 \pm 0.23 \times 10^{-5}$ & $4.05 \pm 1.03 \times 10^{-5}$ \\
$\mathrm{Sb}$ & $2.68 \pm 1.18 \times 10^{-6}$ & $1.65 \pm 0.72 \times 10^{-6}$ \\
$\mathrm{La}$ & $4.02 \pm 1.36 \times 10^{-5}$ & $1.42 \pm 0.15 \times 10^{-6}$ \\
$\mathrm{Ce}$ & $1.27 \pm 0.28 \times 10^{-5}$ & $3.05 \pm 0.55 \times 10^{-6}$ \\
$\mathrm{Sm}$ & $8.30 \pm 2.60 \times 10^{-7}$ & $1.96 \pm 0.22 \times 10^{-7}$ \\
$\mathrm{~Tb}$ & $<2.1 \pm 0.3 \times 10^{-7}$ & $<3.5 \pm 0.2 \times 10^{-8}$ \\
$\mathrm{Hf}$ & $2.52 \pm 0.89 \times 10^{-6}$ & $1.90 \pm 0.86 \times 10^{-6}$ \\
$\mathrm{Au}$ & $1.11 \pm 0.29 \times 10^{-7}$ & $<6.6 \pm 1.3 \times 10^{-8}$ \\
\hline
\end{tabular}

${ }^{1}$ Eight supersilica tubes washed with reagent-grade $\mathrm{HCl}$ and $\mathrm{HNO}_{3}$ and blown with a flame fed by unfiltered oxygen and methane gas.

2Four supersilica tubes washed with ultrapure $\mathrm{HCl}$ and $\mathrm{HNO}_{3}$, double-distilled from Vycor glass, and blown with a flame fed by filtered oxygen and methane gas. seen among refractory lithophiles in individual inclusions could have arisen. Although the activity coefficients necessary for calculating the distribution of all refractory lithophiles among all possible host phases during condensation are unknown, it has been inferred that $\mathrm{Sr}$ was carried into the inclusions predominantly in melilite, $\mathrm{Ba}$ in an unknown phase other than melilite (GROSSMAN et al., 1977), and REEs, $\mathrm{Sc}, \mathrm{Th}, \mathrm{Zr}, \mathrm{Ta}$, and $\mathrm{Hf}$ in one or more of hibonite, perovskite, and zirconium oxide (DAVIS et al., 1978; FAHEY et al., 1987; HINTON et al., 1988).

Although a given pair of refractory lithophiles may be fractionated from one another by relatively large amounts in individual Group I or modified Group I inclusions compared to $\mathrm{C} 1$ chondrites, the fractionation between the same pair of elements is always less than $\sim 20 \%$ when the compositions of large numbers of such inclusions are averaged. This suggests that each refractory lithophile was completely condensed into the assemblage of grains from which this population of refractory inclusions accreted. An exception seems to be $\mathrm{V}$, as the mean $\mathrm{V} / \mathrm{Yb}$ ratio of Group I and modified Group I Allende inclusions is strongly sub-chondritic.

Seven such inclusions analyzed by Grossman et al. (unpubl. data) have a mean $\mathrm{V} / \mathrm{Yb}$ ratio of $0.67 \pm 0.11 \times \mathrm{Cl}$, and two others analyzed by CONARD (1976) have $\mathrm{V} / \mathrm{Yb}$ ratios of $0.66 \pm 0.02$ and $0.58 \pm 0.01$ $\times \mathrm{Cl}$. Assuming $\mathrm{V}$ and $\mathrm{Yb}$ formed ideal solid solutions in precursor phases of Allende inclusions, the condensation or evaporation temperature of $\mathrm{V}$ would have been nearly identical to that of $\mathrm{Yb}$ (DAVIS et al., 1982), and $V$ and $Y b$ would be expected to be present in nearly chondritic proportions in those inclusions. Since, instead, Allende inclusions have strongly sub-chondritic $\mathrm{V} / \mathrm{Yb}$ ratios, it seems likely that $\mathrm{V}$ was slightly more volatile than predicted by the above cal- 
culations such that it was not fully condensed into inclusion precursors at the accretion temperatures of most inclusions.

Refractory siderophiles are enriched by a factor of $\sim 16$ in Allende Group I and modified Group I inclusions relative to $\mathrm{C} 1$ chondrites and, in almost every inclusion, enrichment factors for $\mathrm{Re}, \mathrm{Os}, \mathrm{Ru}$, and Ir differ from one another by less than 20\% (GROSSMAN et al., 1977; MASON and TAYLOR, 1982). In some of these inclusions, W and Mo have enrichment factors that are within $20 \%$ of those for $\mathrm{Re}, \mathrm{Os}$, Ir, and Ru but, in others, refractory siderophile enrichment patterns exhibit negative Mo anomalies, in some cases accompanied by smaller, negative $W$ anomalies. For instance, FEGLEY and PALME (1985) noted that five Allende inclusions with $\mathrm{C} 1$ chondrite-normalized Mo/Os ratios of $0.75,0.71,0.61,0.40$, and $<0.06$ had corresponding $\mathrm{Cl}$ chondrite-normalized $W / O$ s ratios of $0.85,0.87,0.85$, 1.1 , and 0.18 . Refractory siderophiles are now concentrated in tiny, metallic Fe- and Ni-rich opaque assemblages in Allende inclusions, but they are thought to have originally entered those inclusions in condensate metal alloy grains. During CAI fusion, the metal grains melted to form Fe-, Ni-, P-, refractory siderophile element-rich droplets that were immiscible in the co-existing silicate liquid. Upon cooling, those droplets crystallized, new siderophile element alloys formed by exsolution and these assemblages were oxidized to produce the observed opaque assemblages (BLUM et al., 1989).

The simplest interpretation of Allende inclusions which are uniformly enriched in $\mathrm{Re}, \mathrm{Os}, \mathrm{Ru}, \mathrm{Ir}, \mathrm{W}$, and $\mathrm{Mo}$ is that each inclusion sampled grains of a common metal alloy into which each of Re, Os, $\mathrm{Ru}, \mathrm{Ir}, \mathrm{W}$, and Mo was completely condensed. FEGLEY and PALME (1985) recognized, however, that if each refractory siderophile condensed into a common alloy sampled by all Allende inclusions, then in inclusions with $W$ and Mo depletions, that alloy must have formod in a gas whose oxygen fugacity was higher than that of a gas of solar composition. In an oxidizing gas, $\mathrm{Mo}$ is the most volatile of the refractory siderophiles, and $W$ is the next most volatile, so a condensate alloy removed from such a gas at temperatures at which $\mathrm{Re}, \mathrm{Os}, \mathrm{Ru}$, and Ir have completely condensed could have large Mo depletions and smaller $W$ depletions, as could inclusions that preferentially sampled such an alloy.

A different model than that of condensation of refractory siderophiles into a common alloy was proposed by SYLVESTER et al. (1990b). They found that the combination of subchondritic $\mathrm{Re} / \mathrm{Os}$ and $\mathrm{Ir} / \mathrm{Pt}$ ratios in some opaque assemblages from an Allende Group l inclusion cannot be reproduced by the one-phase condensation model, but can be explained by a three-phase model. In the latter, $\mathrm{Re}, \mathrm{Os}$, and $\mathrm{Ru}$, whose high-temperature crystal structures are hexagonal close-packed (hcp), condensed into one alloy, Ir, Pt and Rh, which are face-centered cubic (fcc), condensed into a second, and $\mathrm{W}$ and $\mathrm{Mo}$, which are body-centered cubic (bcc), condensed into a third. Each inclusion sampled large numbers of grains of the three alloys. Thus, fractionations between elements in the same alloy and between elements in different alloys were averaged out to yield chondritic proportions of refractory siderophiles in most bulk inclusions. Inclusions with W and Mo depletions simply may have sampled a ratio of bcc grains to hcp and fec grains that is less than the nebular ratio. According to this model, therefore, such inclusions do not require formation in a highly oxidizing environment, as in the one-phase condensation model.

Some coarse-grained Allende inclusions have relatively unfractionated REE patterns, except for Eu and $\mathrm{Yb}$ anomalies. Such patterns have been called Group III when the Eu and $\mathrm{Yb}$ anomalies are negative and Group VI when they are positive (MASON and TAYLOR, 1982). Inclusions with Group III REE patterns accumulated from condensates or residues that were removed from the nebular gas at a temperature above which Eu and $\mathrm{Yb}$, the most volatile of the REEs, were completely condensed but, in order to account for lack of fractionation among the remaining REEs, the temperature must have been low enough that each of the latter was totally condensed. In contrast, there must be two precursors of inclusions with so-called Group VI REE patterns - one with a Group I pattern and another containing only Eu and $\mathrm{Yb}$. The latter is thought to have condensed from a gas from which material with a Group III pattern was removed previously.

Almost all Allende fine-grained inclusions and a very few coarsegrained ones have Group II REE patterns (GROSSMAN and GANA.
PATHY, 1976b; MASON and TAYLOR, 1982). In such patterns, LREEs and $\mathrm{Tm}$ are comparably enriched to $\sim 20-60$ times their concentrations in $\mathrm{Cl}$ chondrites, other HREEs are progressively depleted with increasing atomic number from $\mathrm{Gd}(10-40 \times \mathrm{Cl})$ to $\mathrm{Lu}(\approx 5 \times \mathrm{C1})$, and $\mathrm{Eu}$ and $\mathrm{Yb}$ exhibit negative anomalies. Enrichment factors for the most refractory $(\mathrm{Hf}, \mathrm{Lu}, \mathrm{Zr}, \mathrm{Sc})$ and most volatile $(\mathrm{V}, \mathrm{Yb}, \mathrm{Eu}$, $\mathrm{Sr}, \mathrm{Ba}$ ) of the refractory lithophiles are smaller than those for refractory lithophiles of intermediate volatility (Th, Tm, La, Ce, Sm, Ta). Refractory siderophiles also have small enrichment factors $(₹ 1 \times C 1)$. Precursors of inclusions with Group II REE patterns condensed from a cooling solar gas after removal of an early, ultra-refractory condensate containing the most refractory of the lithophiles (BOYNTON, 1975; DAVIS and GROSSMAN, 1979) and the refractory siderophiles. The most volatile of the refractory lithophiles are depleted in Group II inclusions because they were only partially condensed at the temperature at which the condensate precursors of these inclusions became isolated from the gas.

IRELAND and FEGLEY (1991) compiled a list of published REE patterns of 108 Allende CAIs. Of the one hundred and eight inclusions, thirty-five (probably mostly fine-grained ones) have Group II REE patterns. Of the remaining seventy-three samples, $78 \%$ have Group I and modified Group I patterns, with and without Eu anomalies, $12 \%$ have Group III patterns, and $8 \%$ have Group VI patterns. One inclusion, HAL, has a LREE-enriched pattern with a large negative Ce anomaly (DAVIS et al., 1982).

\section{GROUP I, TYPE B LEOVILLE AND VIGARANO COARSE-GRAINED INCLUSIONS}

Three Type B, coarse-grained inclusions analyzed in this study, Leo 3537-2, Vig 1623-8, and Vig 1623-11, have Group I or modified Group I REE patterns, which are plotted in Fig. 1. C1 chondrite-normalized refractory lithophile and siderophile element enrichment factors, arranged in order of increasing volatility in a solar gas, are also plotted in this figure. In Fig. 1 and throughout this paper, $\mathrm{C} 1$ chondritenormalization factors are those of ANDERS and GREVESSE (1989), which are given in Table 2. Relative volatilities of refractory lithophiles and siderophiles in a solar gas are those of DAVIS et al. (1982) and SYLVESTER et al. (1990b), respectively, except for Th, which, as noted earlier, seems to have been as volatile as $\mathrm{Ta}, \mathrm{La}$, and $\mathrm{Sm}$ in such a gas.

\section{Leoville 3537-2}

This is a Group I inclusion. $\mathrm{Cl}$ chondrite-normalized REE enrichment factors define a narrow range from $15.6 \pm 0.3$ for Ce to $20.6 \pm 2.5$ for $\mathrm{Tm}$, and most of the other refractory lithophiles and siderophiles have enrichment factors that, within their errors, fall in this range. Exceptions are Hf (27.0 $\pm 0.3)$ and $S c(23.18 \pm 0.03)$, which have anomalously large enrichment factors, and $\mathrm{Th}(10 \pm 1), \mathrm{W}(11.1 \pm 0.5)$, and Mo (6.2 \pm 0.6$)$, which have anomalously small enrichment factors. Anomalies of these sizes for $\mathrm{Hf}, \mathrm{Sc}, \mathrm{Th}, \mathrm{W}$, and Mo are not unusual in individual Allende Group I inclusions, although, in the latter, anomalous enrichment factors for $\mathrm{Hf}$ are smaller than REE enrichment factors, rather than larger, as in Leo 3537-2.

\section{Vigarano 1623-8}

This is a modified Group I inclusion. $\mathrm{Cl}$ chondrite-normalized enrichment factors of LREEs (16.7 \pm 0.1 for $\mathrm{La}$, $13.8 \pm 0.3$ for $\mathrm{Ce}, 15.62 \pm 0.05$ for $\mathrm{Sm}$ ) are lower than those of HREEs (21.0 \pm 0.6 for the mean of Tb, Dy, Ho, Tm, Yb, and $\mathrm{Lu}$ ). Enrichment factors for all other refractory elements 


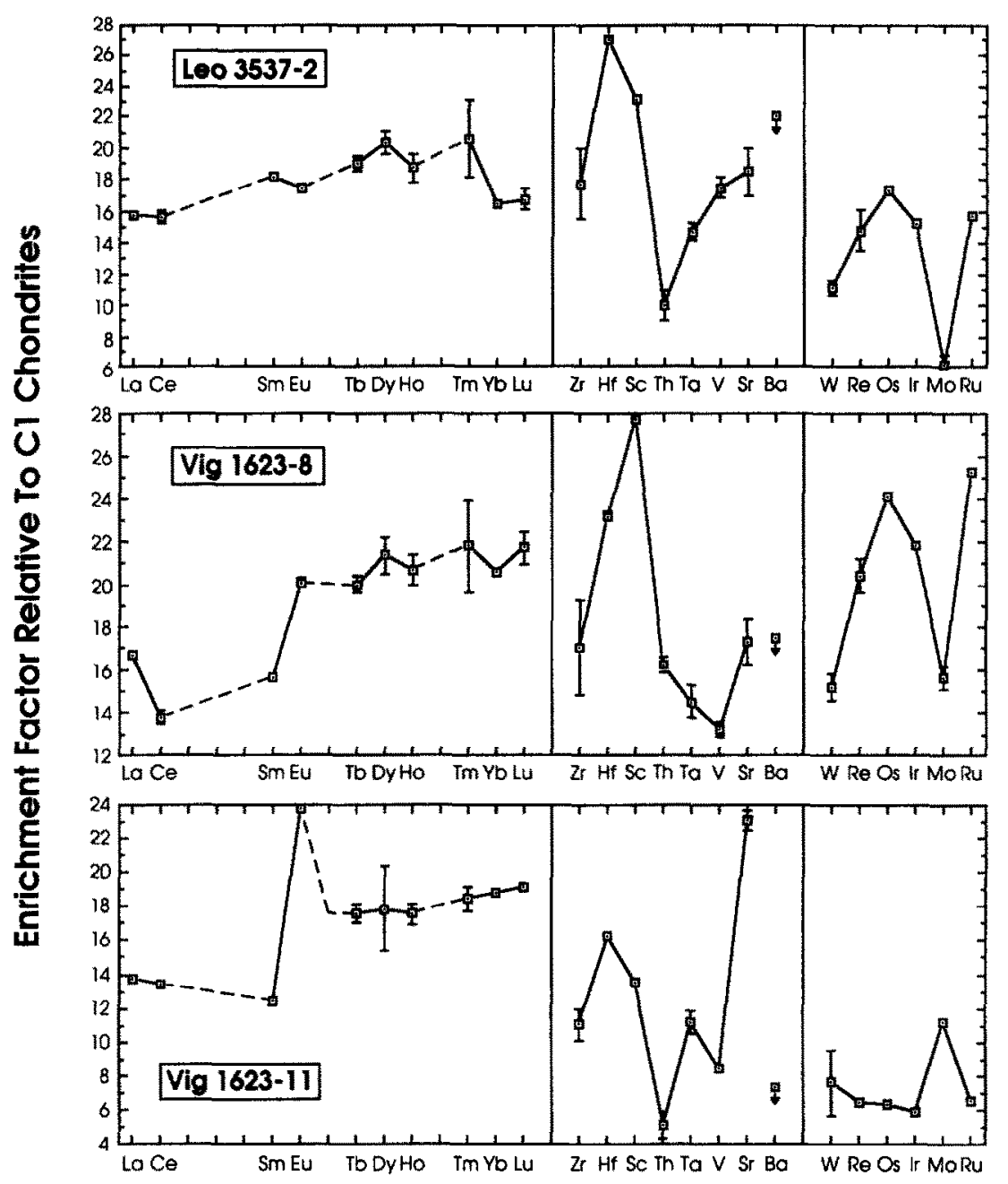

FIG. 1. Cl chondrite-normalized concentrations of REE, refractory lithophiles other than REE and refractory siderophiles in Leo 3537-2, Vig 1623-8, and Vig 1623-11. Refractory lithophiles and siderophiles arranged in order of increasing volatility from left to right. $1 \sigma$ error bars shown where larger than plotted symbols. Arrows indicate $2 \sigma$ upper limits.

are within $20 \%$ of those for HREEs, except that for Sc (27.75 $\pm 0.07 \times \mathrm{C} 1)$, which is larger, and those for Th $(16.3 \pm 0.4)$, $\mathrm{Ta}(14.5 \pm 0.8), \mathrm{V}(13.2 \pm 0.4), \mathrm{W}(15.2 \pm 0.6)$, and Mo $(15.6 \pm 0.5)$, which are similar to enrichment factors for LREEs. The precursors of Vig 1623-8 could have formed by high-temperature condensation or evaporation. Like LREEdepleted, modified Group I inclusions in Allende, a LREEenriched phase, such as hibonite, may have been preferentially excluded, or a HREE-enriched phase, such as zirconium oxide, preferentially included during accretion of the precursors of Vig 1623-8. An alternative explanation for the LREE depletion in Vig 1623-8 is that we inadvertently depleted our analyzed aliquot of the sample in anorthite relative to spinel during removal of material from its host inclusion in the laboratory, as indicated in Table 1. Although anorthite in Type B inclusions is normally enriched in LREEs relative to HREEs (NAGASAwA et al., 1977), preferential sampling of REE-free spinel relative to anorthite would only result in a significant LREE depletion if the anorthite contains a large fraction of the total REEs in the inclusion.

\section{Vigarano 1623-11}

This is an unusual modified Group I inclusion. As in some Allende-modified Group I inclusions, compared to $\mathrm{C} 1$ chondrites, LREEs (mean of $\mathrm{La}, \mathrm{Ce}, \mathrm{Sm}=13.2 \pm 0.5 \times \mathrm{Cl}$ ) are depleted relative to HREEs (mean of $\mathrm{Tb}, \mathrm{Dy}, \mathrm{Ho}, \mathrm{Tm}, \mathrm{Yb}$, $\mathrm{Lu}=18.2 \pm 0.6 \times \mathrm{C} 1)$, enrichment factors for Sc (13.48 $\pm 0.01 \times \mathrm{C} 1)$ and $\mathrm{Hf}(16.3 \pm 0.2 \times \mathrm{C} 1)$ are similar to those of the REEs, and there are anomalously large enrichment factors for $\mathrm{Eu}(23.8 \pm 0.2 \times \mathrm{C} 1)$ and $\mathrm{Sr}(23.1 \pm 0.6 \times \mathrm{C} 1)$. Enrichment factors for refractory siderophiles, except Mo $(11.2 \pm 0.1 \times \mathrm{C} 1)$, are fractionated from one another by less than $20 \%(\mathrm{~W}=7.6 \pm 1.9 \times \mathrm{Cl}, \mathrm{Re}=6.5 \pm 0.2 \times \mathrm{Cl}$, Os $=6.30 \pm 0.08 \times \mathrm{Cl}, \mathrm{Ir}=5.825 \pm 0.004 \times \mathrm{Cl}, \mathrm{Ru}=6.56$ $\pm 0.08 \times \mathrm{Cl}$ ). Vig 1623-11 differs from most modified Group I Allende inclusions, however, in that, compared to $\mathrm{Cl}$ chondrites, $\mathrm{Th}, \mathrm{Ba}$, and refractory siderophiles are strongly depleted relative to REEs ( Th/ $\mathrm{La}=0.37 \pm 0.06 \times \mathrm{Cl}, \mathrm{Ba} / \mathrm{Yb}$ $<0.39 \times \mathrm{Cl}, \mathrm{Os} / \mathrm{La}=0.460 \pm 0.007, \mathrm{Ir} / \mathrm{La}=0.426 \pm 0.003$ $\times \mathrm{Cl}), \mathrm{Zr}$ and $\mathrm{Hf}$ are strongly fractionated from one another 
$(\mathrm{Zr} / \mathrm{Hf}=0.67 \pm 0.06 \times \mathrm{Cl})$, and $\mathrm{Mo}$ is strongly enriched relative to other refractory siderophiles $(\mathrm{Mo} / \mathrm{Ir}=1.92 \pm 0.02$ $X \mathrm{C} 1)$. In fact, Vig 1623-11 has lower $\mathrm{Th} / \mathrm{La}$ and $\mathrm{Ba} / \mathrm{Yb}$ ratios and a higher Mo/Ir ratio than any Allende Group I or modified Group I inclusion, and $\mathrm{Os} / \mathrm{La}, \mathrm{Ir} / \mathrm{La}$ and $\mathrm{Zr} / \mathrm{Hf}$ ratios that are among the lowest in those inclusions. As seen in Table 1, anorthite was preferentially excluded relative to all other phases during laboratory sampling of the aliquot of Vig 1623-11 taken for INAA. Anorthite is not a host for siderophiles and is incapable of fractionating $\mathrm{Zr}$ from $\mathrm{Hf}$. Anorthite in Type B inclusions accounts for only a small fraction of the total Th in these objects but the bulk of the Ba (A. M. Davis, pers. comm.). Thus, nonrepresentative laboratory sampling of Vig 1623-11 may be responsible for the unusually low $\mathrm{Ba} / \mathrm{Yb}$ ratio of this sample but probably not for the other unusual ratios.

During accretion of the precursors of Vig 1623-11, REEbearing phases were preferentially sampled over metal alloy phases and the carrier phase of Th. Since Vig 1623-11 has large enrichment factors for $\mathrm{Eu}$ and $\mathrm{Sr}$, its REE-bearing precursor phases were probably melilite-rich relative to the nebular proportions of these phases. The overall slope of the REE pattern of Vig $1623-11$ is probably due to preferential sampling of HREE-enriched precursor phases, such as $\mathrm{ZrO}_{2}$, over LREE-enriched ones, such as perovskite. These two phases, though minor in abundance, may have contributed more trivalent REEs to most CAls than did melilite. The super-chondritic Mo/Ir ratio of Vig 1623-11 can be understood in the context of the three-phase condensation model of SYLVESTER et al. (1990b) as the result of sampling a greater proportion of bcc alloy grains to fec alloy grains than was generally present in the nebula. In the one-phase condensation model, the combination of a super-chondritic $\mathrm{Mo} / \mathrm{Ir}$ ratio and chondritic proportions of all other siderophiles necessitates mixing of two condensate components. In this model, a super-chondritic $\mathrm{Mo} / \mathrm{Ir}$ ratio arises from condensation in an oxidizing gas from which a higher temperature, Mo-deficient condensate alloy was removed previously. Because of significant volatility differences among the remaining refractory siderophiles, however, there is no temperature at which the early condensate can be removed which will yield a subsequent condensate containing both a super-chondritic $\mathrm{Mo}$ / Ir ratio and chondritic proportions of the remaining refractory siderophiles. Thus, in the one-phase model, nearly pure Mo must be mixed with another phase into which all other refractory siderophiles were completely condensed in order to obtain the refractory siderophile enrichment pattern of Vig 1623-11.

The origin of the strongly sub-chondritic $\mathrm{Zr} / \mathrm{Hf}$ ratio of Vig 1623-11 is unknown. These elements cannot be strongly fractionated from one another on the basis of volatility differences in a solar gas because they have similar condensation temperatures in such a gas (DAvis et al., 1982). After condensation, melting, and crystallization, $\mathrm{Zr}$ and $\mathrm{Hf}$ are concentrated in fassaite in Allende Type B inclusions. Fassaite that crystallized early from a melt having the composition of an Allende Type $\mathrm{B} C \mathrm{CAI}$ with $\mathrm{Cl}$ chondritic proportions of $\mathrm{Zr}$ and $\mathrm{Hf}$ has a $\mathrm{Zr} / \mathrm{Hf}$ ratio of $\sim 0.7 \times \mathrm{Cl}$, whereas later crystallizing fassaite has greater $\mathrm{Zr} / \mathrm{Hf}$ ratios (SIMON et al., 1991). Thus, assuming that fassaite compositions are similar in Vigarano and Allende Type B CAIs, a sub-chondritic $\mathrm{Zr}$ / Hf ratio could have been produced in our sample of Vig 1623-11 by inadvertently enriching it in early fassaite relative to late fassaite during laboratory sampling. We do not think that this occurred, however, because early fassaite in Allende Type B CAIs has a super-chondritic Sc/Lu ratio, $\sim 5 \times \mathrm{Cl}$ (SIMON et al., 1991), whereas our sample has a sub-chondritic $\mathrm{Sc} / \mathrm{Lu}$ ratio, $0.702 \pm 0.007 \times \mathrm{Cl}$.

\section{OLIVINE-RICH VIGARANO INCLUSIONS}

Refractory lithophile and siderophile element enrichment factors in Vig 1623-5, -10, and -13 are plotted in Fig. 2. Compared to their Allende counterparts, Vig 1623-10 and -13 exhibit unusual refractory element fractionations.

\section{Vigarano 1623-10}

This olivine-rich (80\%) aggregate is similar to Allende amoeboid olivine aggregates (GROSSMAN et al., 1979) in that it is uniformly diluted in refractory elements compared to Allende Group I coarse-grained inclusions. The mean enrichment factor for $\mathrm{Ca}, \mathrm{Sc}, \mathrm{Hf}, \mathrm{La}, \mathrm{Ce}, \mathrm{Sm}, \mathrm{Eu}, \mathrm{Tb}, \mathrm{Yb}, \mathrm{Lu}$, $\mathrm{Os}, \mathrm{Ir}$, and $\mathrm{Ru}$ is $4.1 \pm 0.4 \times \mathrm{Cl}$ chondrites in Vig 1623-10, $3.9 \pm 0.2 \times \mathrm{C} 1$ in the average of seven Allende amoeboid olivine aggregates (GROSSMAN et al., 1979), and $18.3 \pm 0.6$ $\times \mathrm{Cl}$ in the average of nine Allende coarse-grained inclusions (Grossman et al., 1977). The uniform dilution of refractory elements in Vig 1623-10, like in Allende amoeboid olivine aggregates, is probably the result of mixing Group I CAI-like condensates or residues with refractory element-poor, and hence lower temperature, olivine-rich material. If each of the aforementioned refractory elements were carried into Vig 1623-10 in CAI-like constituents that were enriched in refractories by a factor of eighteen relative to $\mathrm{Cl}$ chondrites, then the latter comprise $4.1 / 18$ or $23 \%$ of the inclusion, and the remaining $77 \%$ must be lower temperature, olivine-rich constituents.

There are three refractory element characteristics of Vig 1623-10 that are rare in Allende inclusions. First, the $\mathrm{Cl}$ chondrite-normalized enrichment factor of Ir, $4.624 \pm 0.004$, is 1.7 times the mean enrichment factor of $\mathrm{Re}, \mathrm{Os}$, and $\mathrm{Ru}$, $2.7 \pm 0.5$, whereas in Allende amoeboid olivine aggregates, refractory siderophile enrichment factors seldom differ by more than 20\% from one another (GROSSMAN et al., 1979). Enrichment of Ir relative to $\mathrm{Re}, \mathrm{Os}$, and Ru may reflect preferential sampling of fcc alloy grains over hcp grains in the nebula. Second, $\mathrm{Sr}$ and Eu are strongly fractionated from each other ( $\mathrm{Sr} / \mathrm{Eu}<0.12 \times \mathrm{C} 1$ chondrites) in Vig 1623-10 Although the $\mathrm{Sr} / \mathrm{Eu}$ ratios of Allende amoeboid olivine aggregates are not well known, these elements tend to be unfractionated in coarse-grained Allende Group I inclusions, presumably because both elements enter those inclusions in the same condensate phase, melilite, and both are totally condensed into that phase (GrosSMAN et al., 1977). In Vig 1623-10, however, either melilite grains were removed from the nebular gas at a temperature higher than necessary for total condensation of $\mathrm{Sr}$, which may have a slightly lower condensation temperature than $\mathrm{Eu}$ in a gas of solar composition (DAVIS et al., 1982), or $\mathrm{Sr}$ was concentrated into a phase other than melilite, and that phase was not well sampled 


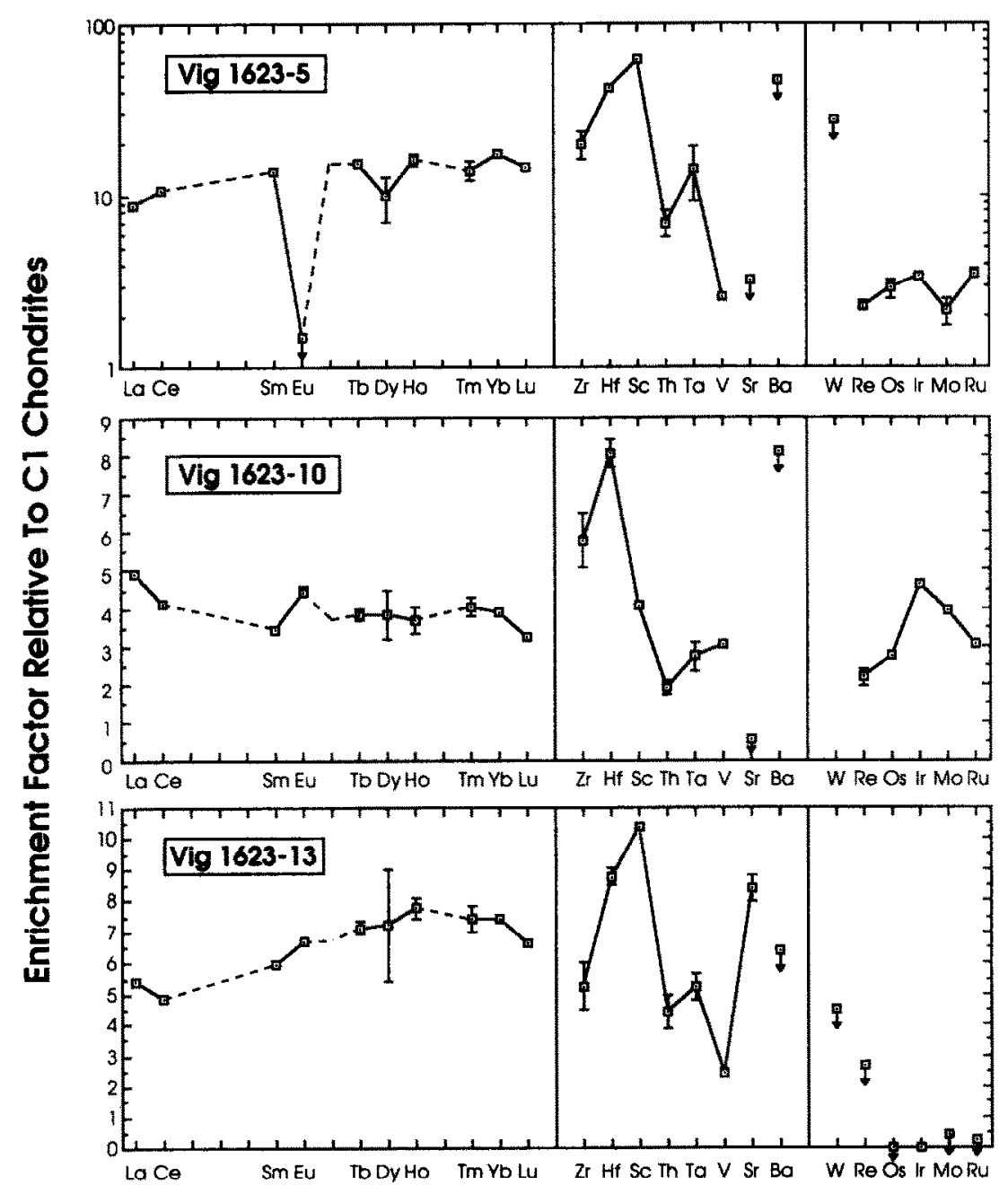

FIG. 2. C1 chondrite-normalized concentrations of REE, refractory lithophiles other than REE and refractory siderophiles in Vig 1623-5, Vig 1623-10, and Vig 1623-13. Refractory lithophiles and siderophiles arranged in order of increasing volatility from left to right. $1 \sigma$ error bars shown where larger than plotted symbols. Arrows indicate $2 \sigma$ upper limits.

by Vig 1623-10. Third, the Th/La ratio of Vig 1623-10 is strongly sub-chondritic $(0.38 \pm 0.04 \times \mathrm{Cl}$ chondrites $)$, suggesting that $\mathrm{Th}$ was not completely condensed into the phases that carried REEs into this inclusion.

\section{Vigarano 1623-13}

This barred olivine chondrule is similar to Allende olivine chondrules in that it is rather uniformly enriched in refractory lithophiles relative to $\mathrm{Cl}$ chondrites, but depleted in those elements relative to Allende Group I inclusions. Refractory lithophile element enrichment factors in Vig 1623-13 are at the high end of the range of those in individual Allende olivine chondrules, however. For instance, the mean enrichment factor for $\mathrm{Ca}, \mathrm{Al}, \mathrm{Sc}, \mathrm{La}, \mathrm{Sm}, \mathrm{Eu}$, and $\mathrm{Yb}$ is $7.5 \pm 0.6$ in Vig 1623-13, ranges from $1.3 \pm 0.1$ to $8 \pm 1$ in twelve Allende olivine chondrules analyzed by RUBIN and WASSON (1987), and is $2.8 \pm 0.1$ in the average of the latter. Like Allende chondrules, Vig 1623-13 probably crystallized from a melt of precursors that included both Group I CAI-like material and material that ceased chemical communication with the nebular gas at lower temperature. If refractory lithophiles in the CAI-like component were enriched by a factor of 18 relative to $\mathrm{Cl}$ chondrites, then that component constitutes 7.5 / 18 or $42 \%$ of the Vig $1623-13$ precursors.

In the Allende olivine chondrules analyzed by RUBIN and WASSON (1987), the lowest CI chondrite-normalized Ir enrichment factor is 0.04 . Vig $1623-13$ is unusual in that its refractory siderophile element enrichment factors are extremely low, even compared to Allende chondrules: Ir $=0.0026 \pm 0.0004, \operatorname{Re}<2.7$, Os $<0.023$, Mo $<0.42$ and $\mathrm{Ru}<0.25$. Allende olivine chondrules tend to have lower enrichment factors for refractory siderophiles than for refractory lithophiles, suggesting that metal and silicate components of those chondrules were fractionated from each other before or during melting. In the Allende olivine chondrules of RUBIN and WASSON (1987), for example, Ir/Sm ratios are sub-chondritic in all but two samples, and as low as $0.02 \times \mathrm{Cl}$. In Vig 1623-13, this ratio is $0.00044 \pm 0.00007$ $\times \mathrm{Cl}$, one of the lowest $\mathrm{Ir} / \mathrm{Sm}$ ratios ever measured in an 
olivine chondrule. Among seventeen such chondrules from the Ornans C3O chondrite, the lowest $\mathrm{Ir} / \mathrm{Sm}$ ratio is 0.15 $\times \mathrm{C} 1$ chondrites (RUBIN and WASSON, 1988), among fifteen from the Qingzhen EH3 chondrite, it is $0.01 \times \mathrm{Cl}$ (GROSSMAN et al., 1985), and among thirty-one from the Semarkona LL3 chondrite, it is $0.01 \times \mathrm{C} 1$, except for two chondrules with Ir concentrations below the detection limit of neutron activation analysis and $\mathrm{Ir} / \mathrm{Sm}$ ratios $<0.003 \times \mathrm{Cl}$ (GROSSMAN and WASSON, 1983, 1985).

Two mechanisms have been suggested for metal/silicate fractionation during chondrule melting. The first is segregation of immiscible siderophile element-rich liquid droplets from silicate liquid and loss of the former from the latter as the chondrule spun in the nebula and the second is loss of siderophiles relative to lithophiles by evaporation. Both mechanisms are thought to have had only a limited role in producing low siderophile element enrichment factors in most chondrules (GROSSMAN and WASSON, 1985). In the case of Vig 1623-13, we have no way of evaluating whether the former mechanism was responsible for loss of refractory siderophiles, but the latter was probably not important. This is because $\mathrm{Eu}, \mathrm{Yb}$, and $\mathrm{Sr}$, which are among the most volatile of the refractory lithophiles in a solar gas, and $\mathrm{Ce}$, which becomes extremely volatile in an oxidizing gas, do not have anomalously low enrichment factors compared to more refractory lithophiles (e.g., $\mathrm{Eu} / \mathrm{La}=1.23 \pm 0.03, \mathrm{Yb} / \mathrm{La}=1.37$ $\pm 0.03, \mathrm{Sr} / \mathrm{La}=1.55 \pm 0.08, \mathrm{Ce} / \mathrm{La}=0.90 \pm 0.02$, relative to $\mathrm{Cl}$ chondrites). It is possible, of course, that the refractory precursors of Vig 1623-13 were simply depleted in refractory siderophiles compared to refractory lithophiles. If all of the Ir in Vig 1623-13 precursors was contained in a CAI-like component which, as discussed above, constituted $42 \%$ of those precursors, then the enrichment factor of Ir was only $0.0026 / 0.42$ or 0.006 in that component relative to $\mathrm{C} 1$ chondrites.

\section{Vigarano 1623-5}

This forsteritic olivine-, fassaite-bearing FUN inclusion described in detail by DAVIS et al. (1991) is thought to have acquired mass fractionated $\mathrm{O}, \mathrm{Mg}$ and $\mathrm{Si}$ isotopes as a result of evaporation, either during or before melting. In our sample, $\mathrm{Cl}$ chondrite-normalized enrichment factors for LREEs (La $=8.7 \pm 0.2 \times \mathrm{C} 1)$ are smaller than those for HREEs (Lu $=14.4 \pm 0.6 \times \mathrm{C} 1), \mathrm{Sc}(61.52 \pm 0.01 \times \mathrm{C} 1)$, and $\mathrm{Hf}(42$ $\pm 2 \times \mathrm{Cl})$, and are greater than those for $\mathrm{Eu}(<1.5 \times \mathrm{Cl})$ and $\operatorname{Sr}(<3.2 \times \mathrm{C1})$. As explained in DAVIS et al. (1991), this composition reflects the fact that, as a result of laboratory sampling, our tiny aliquot of Vig 1623-5 contains a higher proportion of fassaite than does the bulk inclusion. SIMON et al. (1991) showed that partition coefficients for HREEs, $\mathrm{Sc}$, and $\mathrm{Hf}$ are larger, and those for $\mathrm{Eu}$ and $\mathrm{Sr}$ smaller, than those for LREEs in fassaite that crystallized from melts having Type B CAI compositions.

Our sample of Vig 1623-5 has a strongly sub-chondritic $\mathrm{V} / \mathrm{Yb}$ ratio $(0.149 \pm 0.007 \times \mathrm{Cl}$ chondrites), which is probably not the result of preferential fassaite sampling because the bulk $\mathrm{V} / \mathrm{Yb}$ ratio reconstructed from electron and ion microprobe analyses of phases in a thin section of this inclusion is similarly sub-chondritic $(0.110 \pm 0.006 \times \mathrm{Cl}$; DAVIS et al., 1991). Strongly sub-chondritic $\mathrm{V} / \mathrm{Yb}$ ratios arc a com- mon characteristic of Allende FUN inclusions. Such ratios for HAL, CG-14, TE, and C1, for example, are $>0.007$ (HINTON et al., 1988) to <0.31 (DAvis et al., 1982), $0.082 \pm 0.006$ (ClAYTON et al., 1984), $0.084 \pm 0.006$ (WARK et al., 1987), and $\sim 0.02$ (HUTCHEON et al., 1989), respectively. These are lower than even the lowest $\mathrm{V} / \mathrm{Yb}$ ratios found in non-FUN Allende inclusions $(0.22 \times \mathrm{C} 1$; Grossman, unpub. data), and their origin is not understood. They cannot be the result of volatilization and loss of $\mathrm{V}$ during $\mathrm{CAI}$ melting because $\mathrm{Yb}$ and $\mathrm{V}$ have similar volatilities in a solar gas (DAVIS et al., 1982). Vanadium may be lost relative to Yb during evaporation in an oxidizing gas but so should other elements such as $\mathrm{Ce}$, which is depleted neither in Vig $1623-5(\mathrm{Ce} / \mathrm{La}=1.23$ $\pm 0.06 \times \mathrm{Cl}$ chondrites) nor in any FUN inclusion except HAL (DAvis et al., 1982).

C1 chondrite-normalized enrichment factors for Os (2.9 $\pm 0.4)$, $\operatorname{Ir}(3.364 \pm 0.006)$, and $R u(3.5 \pm 0.2)$ in Vig 16235 are within $20 \%$ of one another, but that for $\operatorname{Re}(2.3 \pm 0.2)$ is about $30 \%$ lower than those for $\mathrm{Ir}$ and $\mathrm{Ru}(\mathrm{Re} / \mathrm{Ru}=0.66$ $\pm 0.07 \times \mathrm{Cl} ; \mathrm{Re} / \mathrm{Ir}=0.68 \pm 0.06 \times \mathrm{Cl}$ ). Considering the relatively large errors associated with the low concentrations of refractory siderophiles in Vig 1623-5, the aforementioned $\operatorname{Re}$ fractionation is not much larger than those present in some non-FUN Allende inclusions. Rhenium has been measured in only two Allende FUN inclusions, C1 (HUTCHEON et al., 1989) and TE (WARK et al., 1987). In the latter, Re is depleted by about $30 \%$ relative to Ir and by about $40 \%$ relative to Os, compared to $\mathrm{Cl}$ chondrites.

Although Vig 1623-5 is a FUN inclusion and thus possesses isotopic abundances that are unusual in most Allende inclusions, it exhibits normal refractory element fractionations for non-FUN and FUN Allende inclusions.

\section{GROUP II, FINE-GRAINED VIGARANO INCLUSIONS}

$\mathrm{Cl}$ chondrite-normalized enrichment factors for refractory lithophiles and siderophiles in Vig 1623-14 and -16 are plotted in Fig. 3.

\section{Vigarano 1623-14}

This inclusion has a refractory element composition that is very similar to that of Allende fine-grained inclusions. It has a normal Group II REE pattern: Tm and LREEs are comparably enriched to $\sim 40$ times $\mathrm{Cl}$ chondrites, other HREEs are progressively depleted with increasing atomic number from $\mathrm{Tb}(23.7 \pm 0.2 \times \mathrm{C} 1$ chondrites) to $\mathrm{Lu}(<7.8$ $\times \mathrm{Cl})$, and $\mathrm{Eu}(5.09 \pm 0.12 \times \mathrm{C} 1)$ and $\mathrm{Yb}(6.73 \pm 0.07 \times \mathrm{C} 1)$ have low enrichment factors. The most refractory $(\mathrm{Hf}, \mathrm{Lu}$, $\mathrm{Zr}, \mathrm{Sc}$ ) and most volatile ( $\mathrm{V}, \mathrm{Yb}, \mathrm{Eu}, \mathrm{Sr}, \mathrm{Ba})$ of the refractory lithophiles have lower enrichment factors than those of refractory lithophiles of intermediate volatility $(\mathrm{Th}, \mathrm{Tm}, \mathrm{La}$, $\mathrm{Ce}, \mathrm{Sm}, \mathrm{Ta}$ ). Refractory siderophiles have low enrichment factors, that for Ir being $0.0124 \pm 0.0005$ relative to $\mathrm{C} 1$ chondrites.

\section{Vigarano 1623-16}

This is an unusual Group II inclusion. It is classified as a Group II inclusion because $\mathrm{Cl}$ chondrite-normalized enrichment factors for the most refractory lithophiles (HIf $<0.04$, 


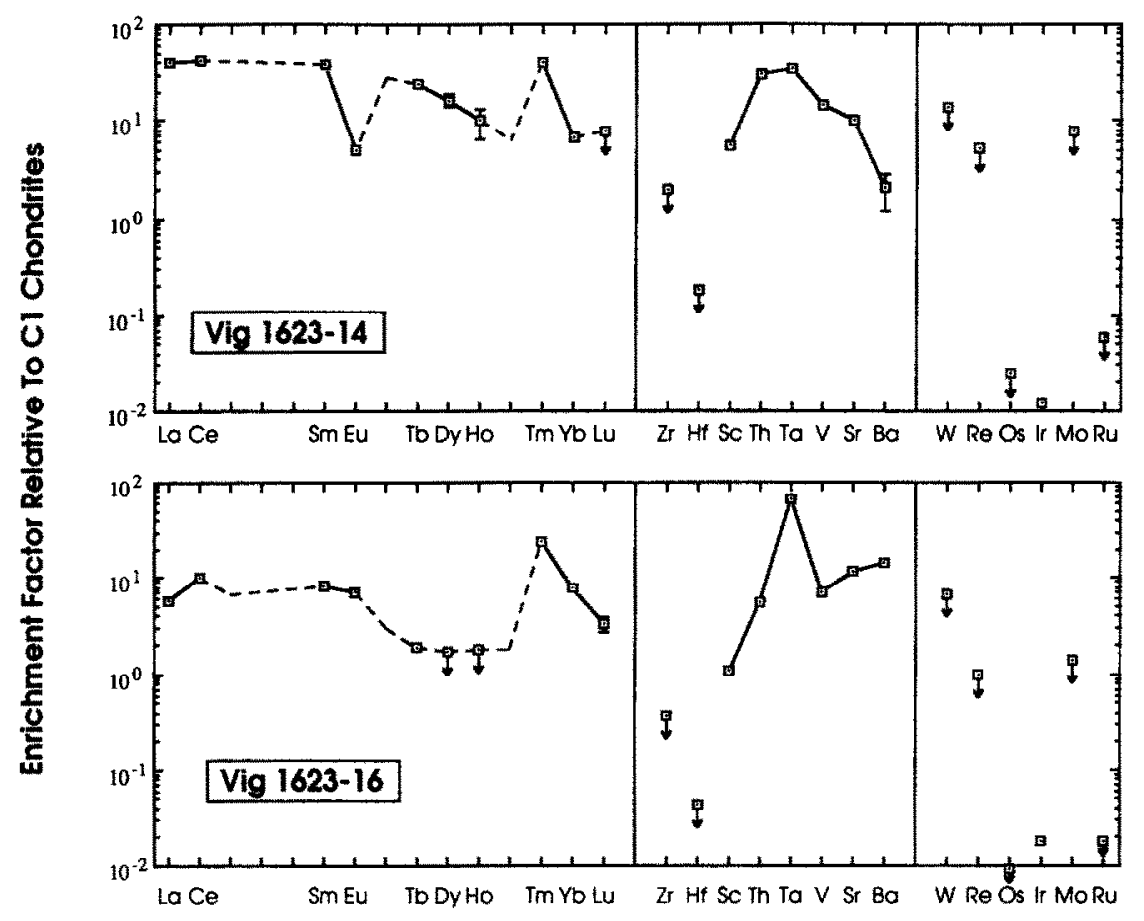

FIG. 3. C1 chondrite-normalized concentrations of REE, refractory lithophiles other than REE and refractory siderophiles in Vig 1623-14 and Vig 1623-16. Refractory lithophiles and siderophiles arranged in order of increasing volatility from left to right. $1 \sigma$ error bars shown where larger than plotted symbols. Arrows indicate $2 \sigma$ upper limits.

$\mathrm{Sc}=1.055 \pm 0.005, \mathrm{Zr}<0.38, \mathrm{Lu}=3.3 \pm 0.6, \mathrm{~Tb}=1.90$ \pm 0.03 , Dy $<1.7$, Ho $<1.8)$ and the refractory siderophiles $(\mathrm{Ir}=0.0183 \pm 0.0002)$ are smaller than those for refractory lithophiles of intermediate volatility $(\mathrm{La}=5.8 \pm 0.1, \mathrm{Sm}$ $=8.27 \pm 0.05, \mathrm{Th}=5.4 \pm 0.1, \mathrm{Tm}=23.6 \pm 0.1, \mathrm{Ta}$ $=65.9 \pm 0.6$ ). It is unusual because enrichment factors for $\mathrm{La}, \mathrm{Sm}$, and $\mathrm{Th}$ are 3 to 10 times smaller than in normal Group II inclusions, and because its REE pattern has a positive $\mathrm{Ce}$ anomaly, with $\mathrm{Ce} / \mathrm{La}=1.72 \pm 0.04$ relative to $\mathrm{Cl}$ chondrites. Inclusions having compositions similar to that of Vig 1623-16 have been referred to as modified Group IIs. Such inclusions are thought to have condensed after an early, ultrarefractory condensate was removed from the nebular gas, as was the case for normal Group II inclusions. In this case, however, the removal temperature of that early condensate was sufficiently low that $T h$ and the most refractory of the LREEs ( $\mathrm{La}, \mathrm{Sm}$ ) were removed along with $\mathrm{Hf}, \mathrm{Zr}, \mathrm{Sc}$, the refractory HREEs, and the refractory siderophiles. Thus, $\mathrm{Th}, \mathrm{La}$, and $\mathrm{Sm}$, which are more refractory than $\mathrm{Ce}$, were depleted relative to $\mathrm{Ce}$ in the gas from which modified Group II inclusions subsequently condensed. The enrichment factor for $\mathrm{Ta}$ is larger than those for LREEs in modified Group II inclusions because $\mathrm{Ta}$ is more volatile than those REEs, resulting in smaller fractions of Ta than LREEs being removed from the nebular gas in the ultrarefractory condensate. Like Vig 1623-16, some modified Group IIs have super-chondritic $\mathrm{Tm} / \mathrm{Sm}$ ratios. In a solar gas and assuming ideal solid solution, Tm is more refractory than Sm (DAvis et al., 1982). Thus, an early ultrarefractory condensate that removed a significant fraction of the $\mathrm{Sm}$ from a cooling solar gas would have removed even more of the $\mathrm{Tm}$, and subsequent condensates would have sub-chondritic $\mathrm{Tm} / \mathrm{Sm}$ ratios. Since Vig 162316 has a super-chondritic $\mathrm{Tm} / \mathrm{Sm}$ ratio, $\mathrm{Tm}$ must have been more volatile than $\mathrm{Sm}$ during condensation of the ultrarefractory precursors of Vig 1623-16. Perhaps those precursors preferentially excluded $\mathrm{Tm}$ relative to $\mathrm{Sm}$ because they consisted of hibonite or perovskite, both of which have strong crystal-chemical preferences for LREEs over HREEs. It is not known whether nonrepresentative laboratory sampling of Vig 1623-16 can be responsible for the unusual Tm/Sm ratio, as the detailed modal mineralogy of this inclusion could not be determined and the distribution of trace elements within fine-grained inclusions is unknown.

Modified Group II inclusions are not common in Allende, with samples A-2 of CONARD (1976), 3803 of MASON and MARTIN (1977), and 5242 of MASON and TAYLOR (1982) being the only unambiguous examples. Allende sample 16 of GROSSMAN and GANAPATHY (1976b) may be another, but this is less clear because its $\mathrm{Tm}$ concentration was not measured. Other reported occurrences of modified Group II inclusions are from four hibonite-bearing CAIs in the unique chondrite ALH85085 (F101, F104, F123 of MACPHERSON et al., 1989; CAI \#174 of El. GORESY et al., 1990b), one Type B2 CAI, Leo 3537-1, in Leoville (MAO et al., 1990), and two CAIs, CL 7-1 and CL9, from the Mokoia C3V chondrite (LIU et al., 1987). IRELAND et al. (1988) and IRELAND (1990) have reported similar compositions in perovskite and hibonite grains from the Murchison $\mathrm{C} 2 \mathrm{M}$ chondrite.

Vig 1623-16 is similar to most normal and some modified (Allende 3803, 5242; Mokoia CL7-1, CL9) Group II CAIs in that it exhibits sub-chondritic Eu/Sm $(0.840 \pm 0.007 \times \mathrm{Cl})$ and $\mathrm{Yb} / \mathrm{Tm}(0.338 \pm 0.002 \times \mathrm{C} 1)$ ratios. Such ratios are the 


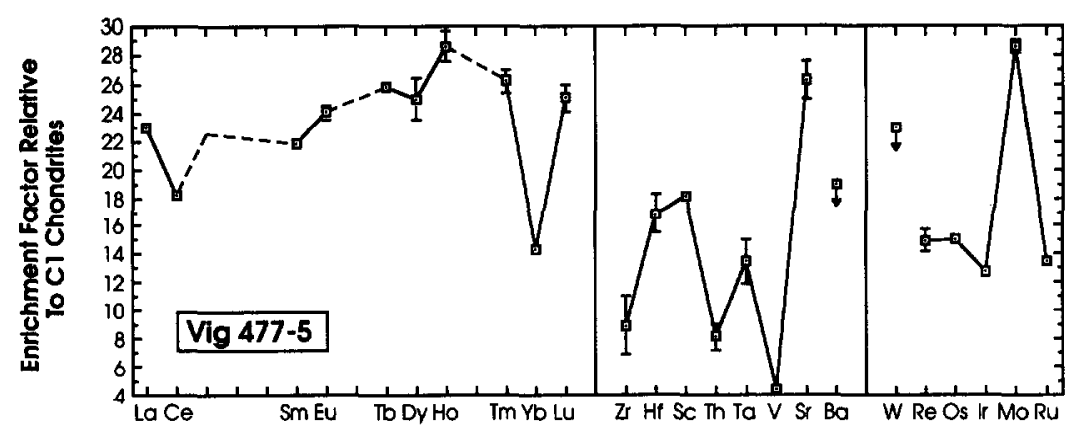

FIG. 4. C1 chondrite-normalized concentrations of REE, refractory lithophiles other than REE and refractory siderophiles in Vig 477-5. Refractory lithophiles and siderophiles arranged in order of increasing volatility from left to right. $1 \sigma$ error bars shown where larger than plotted symbols. Arrows indicate $2 \sigma$ upper limits.

result of removal of the condensate precursors of these inclusions from the cooling nebular gas before $\mathrm{Eu}$ and $\mathrm{Yb}$, the two most volatile REEs, could fully condense into them.

\section{OTHER INCLUSIONS}

\section{Fluffy Type A Vigarano Inclusion}

Vig 477-5 has an unusual REE pattern (Fig. 4). LREEs $(\sim 22 \times \mathrm{Cl}$ chondrites) and HREEs $(\sim 26 \times \mathrm{C} 1)$ are comparably enriched, but there is a small negative Ce anomaly $(\mathrm{Ce} / \mathrm{La}=0.79 \pm 0.01 \times \mathrm{Cl})$, a large negative $\mathrm{Yb}$ anomaly $(\mathrm{Yb} / \mathrm{Lu}=0.57 \pm 0.02 \times \mathrm{C} 1)$, and little or no Eu anomaly $(\mathrm{Eu} / \mathrm{Sm}=1.10 \pm 0.02 \times \mathrm{Cl})$. Fassaite, perovskite, and melilite in Vig 477-5 do not have negative $\mathrm{Ce}$ and $\mathrm{Yb}$ anomalies, according to ion microprobe analyses (DAVIS et al., 1986, 1987). Negative $\mathrm{Ce}$ and $\mathrm{Yb}$ anomalies found in the bulk inclusion, therefore, must reflect the compositions of REEbearing phases other than these minerals, such as hibonite.

There are few refractory inclusions with a REE pattern like that of Vig 477-5. Two inclusions, CG3 from Allende (Grossman and GaNAPATHY, 1976a; Grossman et al., 1977) and CAI \#2 from the Felix C3O chondrite (MISAWA and NAKAMURA, 1988), and a hibonite grain, SHIB 13-24, from Murchison (IRELAND, 1990) have REE patterns with large negative $\mathrm{Yb}$ anomalies and no Eu anomalies, but they do not have negative $\mathrm{Ce}$ anomalies, even small ones like that in Vig 477-5. The REE patterns of two other Murchison hibonite grains, SHIB 7-373 and 7-A95 (IRELAND et al., 1988), do have negative $\mathrm{Ce}$ and $\mathrm{Yb}$ anomalies and no $\mathrm{Eu}$ anomaly, but the $\mathrm{Ce}$ anomaly in the former $(\mathrm{Ce} / \mathrm{La}=0.84 \times \mathrm{Cl}$ chondrites) is smaller than even that in Vig 477-5, and the REE pattern of the latter is HREE-enriched ( $\mathrm{Lu} / \mathrm{La} \sim 5 \times \mathrm{Cl}$ ), whereas Vig 477-5 has a relatively flat REE pattern.

Among the most volatile REEs, Eu is more volatile than $\mathrm{Yb}$ which is more volatile than Ce. Group III REE patterns, characterized by negative Eu and $\mathrm{Yb}$ anomalies, are thought to have formed by removal from the nebular gas at temperatures too high for Eu and $\mathrm{Yb}$ to have completely condensed. Removal at a slightly higher temperature would also produce a small negative $\mathrm{Ce}$ anomaly. Vig 477-5 may have accreted from two components: one that formed at a high enough temperature that it had negative $\mathrm{Ce}, \mathrm{Eu}$, and $\mathrm{Yb}$ anomalies and a component enriched in Eu compared to all other REEs.
The latter could have formed after prior condensation of all REEs except Eu, the most volatile REE.

There are some other unusual refractory element fractionations in Vig 477-5: $\mathrm{Zr} / \mathrm{Lu}$ and $\mathrm{Th} / \mathrm{La}$ ratios are strongly subchondritic, $0.36 \pm 0.09$ and $0.35 \pm 0.04$ relative to $\mathrm{Cl}$ chondrites, respectively, and $\mathrm{Mo} / \mathrm{Ir}$ and $\mathrm{Mo} / \mathrm{Os}$ ratios are strongly super-chondritic, $2.25 \pm 0.03$ and $1.90 \pm 0.04$ relative to $\mathrm{Cl}$ chondrites, respectively. The latter ratios reflect preferential sampling of bcc alloy grains relative to fcc and hcp alloy grains by the inclusion in the nebula. The former ratios suggest that $\mathrm{Zr}$ and $\mathrm{Th}$ were carried in a separate condensate phase from that which carried the REEs, and that Vig 477-5 preferentially sampled the REE carrier relative to the $\mathrm{Zr}$ and $\mathrm{Th}$ carrier. The excellent agreement between calculated and observed modes seen in Table 1 for this inclusion suggests that the unusual $\mathrm{Zr} / \mathrm{Lu}$ and $\mathrm{Th} / \mathrm{La}$ ratios are probably not due to nonrepresentative sampling in the laboratory.

\section{Type B CAI Vigarano 477-B}

This inclusion has a very unusual REE pattern (Fig. 5). Enrichment factors relative to $\mathrm{Cl}$ chondrites are highest for Tm (99 $\pm 2 \times \mathrm{Cl})$, somewhat less for the most refractory of the HREEs ( $\mathrm{Tb}=52.1 \pm 0.8 \times \mathrm{C} 1$, Dy $=63 \pm 2 \times \mathrm{C} 1$, Ho $=57 \pm 2 \times \mathrm{C} 1, \mathrm{Lu}=53.1 \pm 0.8 \times \mathrm{C} 1)$, and even lower for the LREEs ( $\mathrm{La}=24.5 \pm 0.2 \times \mathrm{Cl}, \mathrm{Sm}=25.36 \pm 0.07 \times \mathrm{C} 1)$. There is a small negative $\mathrm{Ce}$ anomaly $(\mathrm{Ce} / \mathrm{La}=0.79 \pm 0.03$ $\times \mathrm{Cl})$, and larger ones for $\mathrm{Eu}(\mathrm{Eu} / \mathrm{Sm}=0.56 \pm 0.01 \times \mathrm{Cl})$ and $\mathrm{Yb}(\mathrm{Yb} / \mathrm{Lu}=0.56 \pm 0.01 \times \mathrm{Cl})$. Refractory lithophiles with the highest condensation temperatures, $\mathrm{Hf}, \mathrm{Lu}, \mathrm{Zr}, \mathrm{Sc}$, $\mathrm{Tb}, \mathrm{Dy}$, and Ho, have a mean enrichment factor of $58 \pm 2$, greater than the mean of $\mathrm{La}, \mathrm{Ce}, \mathrm{Sm}, \mathrm{Th}, \mathrm{Ta}, \mathrm{V}, \mathrm{Yb}, \mathrm{Eu}$, and $\mathrm{Sr}, 23 \pm 2$, which have lower condensation temperatures. Refractory siderophile element enrichment factors are less than those of all refractory lithophiles, with the mean of $\mathrm{Re}$, Os, Ir, and Ru being $13 \pm 1 \times \mathrm{C} 1$, and those for $\mathrm{W}(4.9 \pm 0.6$ $\times \mathrm{C} 1)$ and $\mathrm{Mo}(7.6 \pm 0.7 \times \mathrm{C} 1)$ being even lower.

In Fig. 6, the REE pattern of Vig 477-B is compared to that of a modified Group II inclusion, Vig 1623-16, and that of a hypothetical condensate removed from a solar gas at a temperature just below that at which hibonite appears, 1727 $\mathrm{K}$ at $10^{-3} \mathrm{~atm}$, calculated assuming ideal solid solution (EKAMBARAM et al., 1984) and normalized to $10 \times \mathrm{Cl}$ chondritic $\mathrm{La}$. The latter is enriched in the more refractory REEs (the 


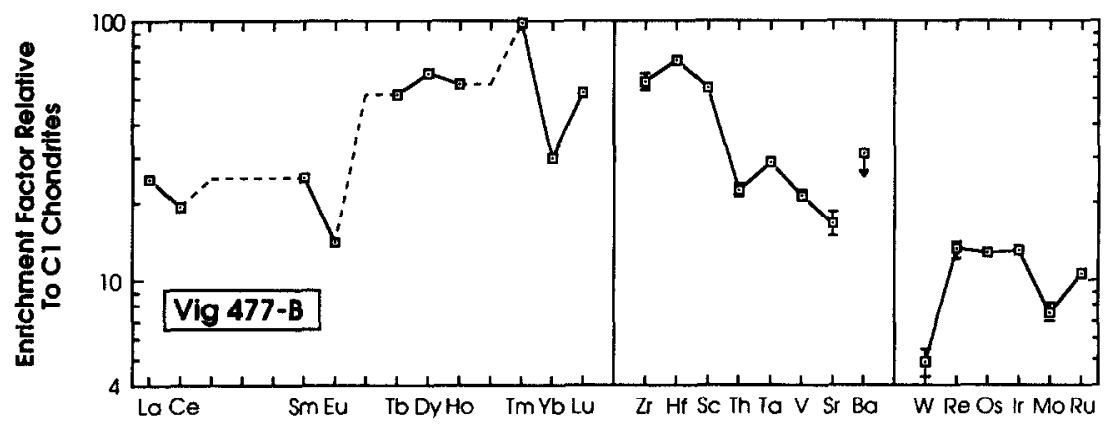

FIG. 5. C1 chondrite-normalized concentrations of REE, refractory lithophiles other than REE and refractory siderophiles in Vig 477-B. Refractory lithophiles and siderophiles arranged in order of increasing volatility from left to right. $1 \sigma$ error bars shown where larger than plotted symbols. Arrow indicates $2 \sigma$ upper limit.

heavy ones except $\mathrm{Yb}$ ) relative to the more volatile REEs (the light ones, Eu and $\mathrm{Yb}$ ), which had not completely condensed at the temperature of removal, and is thus referred to as an ultrarefractory condensate. The REE pattern of Vig 477-B is similar to that of the ultrarefractory condensate in being enriched in more refractory HREEs relative to more volatile LREEs, but differs in having smaller negative Eu and $\mathrm{Yb}$ anomalies and a sub-chondritic $\mathrm{Lu} / \mathrm{Tm}$ ratio. Small Eu and $\mathrm{Yb}$ anomalies and a sub-chondritic $\mathrm{Lu} / \mathrm{Tm}$ ratio are characteristics of many Group II inclusions, suggesting that the REE pattern of Vig 477-B can be explained as a mixture of an ultrarefractory REE pattern and a Group II one. In Vig $477-\mathrm{B}$, the high $\mathrm{Tb} / \mathrm{Sm}$ ratio of 2.0 relative to $\mathrm{Cl}$ chondrites requires a relatively large contribution from the ultrarefrac-

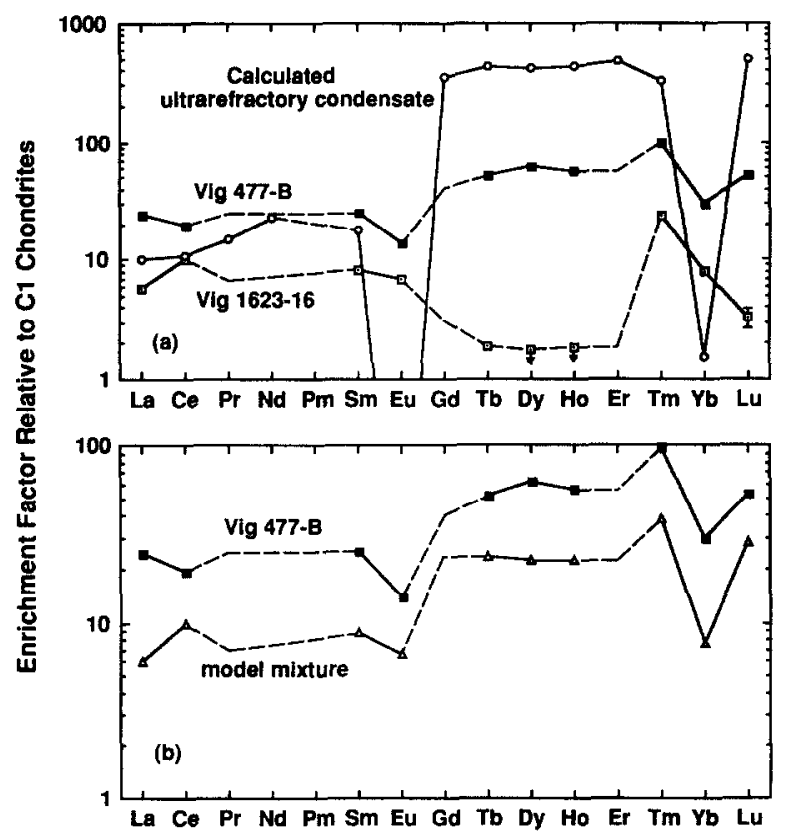

FIG. 6. Mixing models for Vig 477-B. (a) Cl chondrite-normalized concentrations of REE in Vig 477-B, a calculated ultrarefractory condensate, and Vig 1623-16, which has a modified Group II pattern. (b) $\mathrm{Cl}$ chondrite-normalized concentrations of REEs in a model mixture ( $5 \%$ ultrarefractory condensate plus $95 \%$ Vig 1623-16) compared to those of Vig 477-B. tory component which is enriched in HREEs relative to LREEs compared to $\mathrm{C} 1$ chondrites, while the opposite is true for the Group II component. At the same time, the high Tm/ $\mathrm{Tb}$ ratio of 1.9 in $\mathrm{Vig} 477-\mathrm{B}$ relative to $\mathrm{Cl}$ chondrites requires a relatively large contribution from the Group II component whose $\mathrm{Tm} / \mathrm{Tb}$ ratio is super-chondritic, while that of the ultrarefractory component is sub-chondritic. Although these two ratios in Vig 477-B cannot be satisfied simultaneously by mixing an ultrarefractory component with a normal Group II one, they can be satisfied if a modified Group II component is used, since some modified Group II inclusions, like Vig 1623-16, have much higher $\mathrm{Cl}$ chondrite-normalized $\mathrm{Tm} /$ $\mathrm{Tb}$ ratios than normal Group IIs, requiring much less of a contribution from the Group II component and thus maintaining a super-chondritic $\mathrm{Tb} / \mathrm{Sm}$ ratio in the mixture. Mixing $5 \%$ of the ultrarefractory condensate shown in Fig. 6a and 95\% of modified Group II inclusion Vig 1623-16, for example, produces a model REE pattern similar in shape to that of Vig 477-B, as shown in Fig. 6b. Both are enriched in HREEs, except $\mathrm{Yb}$, relative to LREEs, have sub-chondritic $\mathrm{Lu} / \mathrm{Tm}$ ratios and super-chondritic $\mathrm{Tm} / \mathrm{Sm}$ ratios, and exhibit negative $\mathrm{Eu}$ and $\mathrm{Yb}$ anomalies. The model mixture has a positive Ce anomaly, unlike Vig 477-B, indicating that the modified Group II component in Vig 477-B differed from its counterpart in Fig. 6 in having a negative Ce anomaly. Such a Ce anomaly could have formed in the modified Group II component if the latter condensed from the nebular gas at a higher temperature than its counterpart used in the mixing calculation. At higher temperatures, smaller fractions of $\mathrm{Ce}$ would have condensed than of the other LREEs, since $\mathrm{Ce}$ is the most volatile of those REEs.

If a modified Group II component with the composition of Vig 1623-16 makes up 95\% of Vig 477-B, then, by mass balance, the ultrarefractory component comprising the remaining 5\% must have $\mathrm{Cl}$ chondrite-normalized enrichment factors of 1400 for $\mathrm{Hf}, 1160$ for $\mathrm{Zr}, 1080$ for Sc, 350 for $\mathrm{Th}$, 300 for $\mathrm{V}, 110$ for $\mathrm{Sr}, 260$ for Ir and Os, 210 for $\mathrm{Ru}$, and $\sim 260$ for Re. Larger enrichment factors are expected in the ultrarefractory component of Vig 477-B for $\mathrm{Hf}, \mathrm{Zr}$, and Sc than for $\mathrm{Th}, \mathrm{V}$, and $\mathrm{Sr}$ because the former are more refractory than the latter. In ultrarefractory inclusion SH-2 from Murchison, for example, $\mathrm{C} 1$ chondrite-normalized enrichment factors are 1800 for $\mathrm{Hf}, 1200$ for $\mathrm{Zr}, 260$ for $\mathrm{Sc},<180$ for Th, $<11$ for $\mathrm{V}$, and $<218$ for Sr (EKAMBARAM et al., 1984). 
The modified Group II component of Vig 477-B must have a lower Ta concentration than Vig 1623-16 because the latter requires the ultrarefractory component of Vig 477-B to have a Ta concentration that is less than zero. It is plausible for the modified Group II component of Vig 477-B to have the same REE concentrations as Vig 1623-16 while having a lower Ta concentration because $\mathrm{Ta} / \mathrm{La}$ ratios of some modified Group II inclusions are lower than that of Vig 1623-16. In the latter, the $\mathrm{Ta} / \mathrm{La}$ ratio is $11.4 \times \mathrm{Cl}$ chondrites, but in Mokoia inclusion CL7-1 (LIU et al., 1987), the Ta/La ratio is $3.0 \times \mathrm{Cl}$, and in Allende inclusion A-2 (CONARD, 1976), it is $1.3 \times \mathrm{C} 1$. In a solar gas, Ta is about as refractory as $\mathrm{Ce}$, and slightly more volatile than $\mathrm{La}$ and Sm (DAVIS et al., 1982). Thus, $\mathrm{Ta} / \mathrm{La}$ ratios are predicted to be lowest in modified Group II inclusions whose condensate constituents were removed from the nebular gas at unusually high temperatures, as seems to be indicated for the Group II component of Vig 477-B by the $\mathrm{Ce}$ content of the latter. If the modified Group II component of Vig 477-B has a $\mathrm{Ta} / \mathrm{La}$ ratio like that of CL7-1, 3.0 $\times \mathrm{Cl}$, then the enrichment factor for Ta in the modified Group II component of Vig $477-\mathrm{B}$ is $\sim 17 \times \mathrm{Cl}$, and the ultrarefractory component of Vig 477-B has an enrichment factor for Ta of $250 \times \mathrm{Cl}$ chondrites.

Compared to ultra-refractory inclusion SH-2, which has enrichment factors for Ir, Os, Ru, and Re of $1696 \pm 4,3100$ $\pm 16,162 \pm 13$, and $3808 \pm 30 \times \mathrm{Cl}$ chondrites, respectively, and to ultrarefractory inclusion RNZ from the Ornans C3O chondrite (PALME et al., 1982), which has enrichment factors for Ir, Os, Ru, and Re of $14490 \pm 440,15210 \pm 760,3760$ \pm 750 , and $15260 \pm 460 \times \mathrm{Cl}$ chondrites, respectively, the ultrarefractory component of Vig 477-B has small enrichment factors for refractory siderophiles and, therefore, must have sampled smaller amounts of siderophile element-rich metal alloys than did SH-2 and RNZ. Also, refractory siderophiles must have condensed into the metal alloys that contributed to the ultrarefractory component of Vig 477-B at lower temperatures than into those alloys that contributed to $\mathrm{SH}-2$ and RNZ. This is because SH-2 and RNZ have strongly superchondritic $\mathrm{Os} / \mathrm{Ru}$ ratios of $19 \pm 2$ and $4.0 \pm 0.8 \times \mathrm{Cl}$ chondrites, respectively, whereas the ultrarefractory condensate of Vig 477-B has only a slightly super-chondritic Os/Ru ratio of $1.24 \pm 0.05 \times \mathrm{C} 1$. Since Os is much more refractory than $\mathrm{Ru}$ in a solar gas, alloys condensing from such a gas have strongly super-chondritic Os/Ru ratios at high temperatures and $O s / R u$ ratios that approach unity at lower temperatures. According to the threc-phase condensation model of SYLVESTER et al. (1990b), for example, hcp condensate alloy grains of the ultrarefractory component of Vig 477-B were removed from the nebular gas at $1575 \mathrm{~K}$ and $10^{-3} \mathrm{~atm}$, whereas those of RNZ were removed at $1640 \mathrm{~K}$ and $10^{-3}$ atm. The foregoing conclusions are not changed if the modified Group II component of Vig 477-B is assumed to have the Re, Os, Ir, and Ru concentrations of a modified Group II inclusion other than Vig 1623-16. For instance, if the modified Group II component of Vig 477-B has the refractory siderophile element concentrations of Leo 3537-1 (MAO et al., 1990), the ultrarefractory component of Vig 477-B will have $\mathrm{C} 1$ chondrite-normalized enrichment factors of 250 for Ir, Os, and Re and 190 for $\mathrm{Ru}$, and an Os/Ru ratio of 1.29 $\pm 0.05 \times \mathrm{C} 1$.

\section{NON-REFRACTORY ELEMENT CONCENTRATIONS}

Non-refractory elements ( $\mathrm{Ni}, \mathrm{Co}, \mathrm{Mg}, \mathrm{Fe}, \mathrm{Si}, \mathrm{Cr}, \mathrm{Au}, \mathrm{Mn}$, As, $\mathrm{K}, \mathrm{Na}, \mathrm{Ga}, \mathrm{Sb}, \mathrm{Cl}, \mathrm{Se}, \mathrm{Cs}, \mathrm{Zn}, \mathrm{Br}$ ) are thought to have been added to CAIs during low-temperature $(<1470 \mathrm{~K}$ at $10^{-3} \mathrm{~atm}$ for $\mathrm{Ni}, \mathrm{Co}, \mathrm{Mg}, \mathrm{Si}, \mathrm{Cr}, \mathrm{Au}, \mathrm{Mn}, \mathrm{As}$ and some of the $\mathrm{Fe} ;<1100 \mathrm{~K}$ for the others) reaction with the nebular gas, before the inclusions were incorporated into their meteorite hosts. As a result of low-temperature alteration, secondary mineral phases formed in the inclusions. Previous work suggests that coarsc-graincd inclusions from the reduced subgroup of C3V chondrites, including Vigarano and Leoville, experienced secondary alteration at higher temperatures or for a shorter time than those from the oxidized subgroup, which includes Allende (MAO et al., 1990). This is because the former tend to have lower concentrations of non-refractory elements, particularly $\mathrm{Na}$ and $\mathrm{Au}$ (plotted in Fig. 7a), than do their counterparts in the oxidized subgroup (BIsCHOFF et al., 1987; MAO et al., 1990). Also, low-temperature, secondary mineral phases are much less abundant in CAIs from Vigarano than in those from Allende (MACPHERSON, 1985). At high temperatures, non-refractory elements were not completely condensed from the nebular gas, and inclusions bathed in that gas were less likely to acquire high concentrations of those elements, and mineral phases composed of them, than were inclusions immersed in a low-temperature gas. Similarly, the shorter the time an inclusion was exposed to nebular gas at low temperature, the less likely was it that non-refractory elements and their mineral phases were deposited in that inclusion.

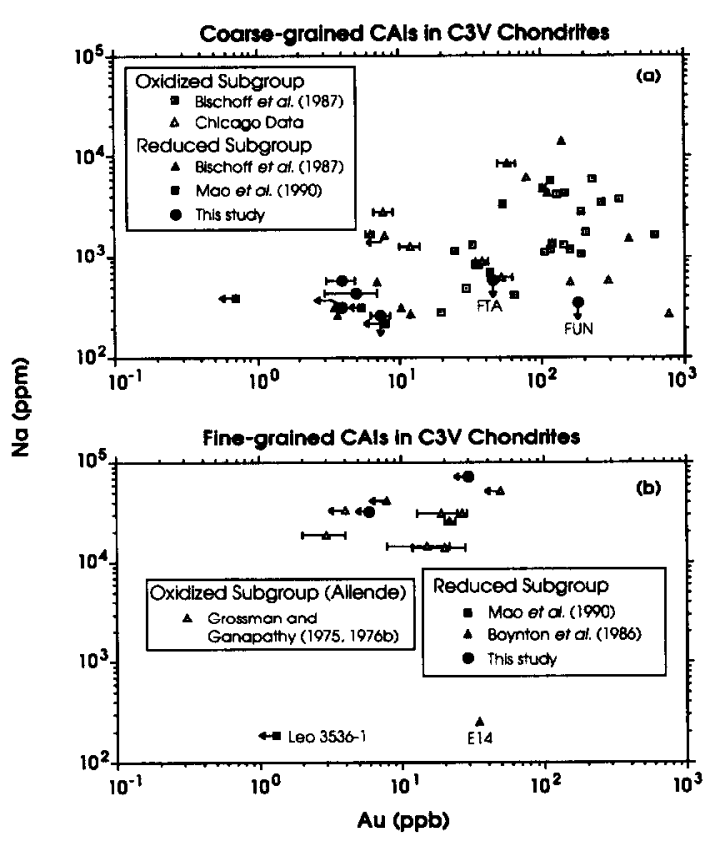

FiG. 7. Concentrations of $\mathrm{Na}$ and $\mathrm{Au}$ in (a) coarse-grained and (b) fine-grained CAIs of the oxidized and reduced subgroups of C3V chondrites. FTA = fluffy Type A inclusion, Vig 477-5 (this study). FUN = Vig 1623-5 (this study). Data for Leo 3536-1 from MAO et al. (1990) and for E14 from BoYNTON et al. (1986). $1 \sigma$ error bars shown where larger than plotted symbols. Arrows indicate $2 \sigma$ upper limits. 
The relatively low concentrations of $\mathrm{Na}$ and $\mathrm{Au}$ (Fig. 7a) and the relatively low amounts of secondary mineral phases in the coarse-grained Vigarano and Leoville CAIs studied here are consistent with the idea that coarse-grained inclusions in the reduced subgroup of $\mathrm{C} 3 \mathrm{~V}$ chondrites were altered at higher temperatures or for a shorter lime than those in the oxidized subgroup. Two of the Vigarano CAIs, however, are anomalously enriched in Au compared to all other CAIs from the reduced subgroup. One is the fluffy Type $A$ inclusion, Vig 477-5, which has $0.33 \pm 0.02 \times \mathrm{Cl}$ chondritic $\mathrm{Au}$, and the other is the olivine-rich, FUN inclusion, Vig 1623-5, which has $1.26 \pm 0.02 \times \mathrm{Cl}$ chondritic Au. Since Au has a higher condensation temperature than Na (PALME et al., 1988), Vig 477-5 and 1623-5 must have been altered at a lower temperature than all other CAIs of the reduced subgroup, so that Au condensed in significant amounts, but at a high enough temperature that $\mathrm{Na}$ did not condense in significant amounts, unlike CAIs of the oxidized subgroup.

Unlike $\mathrm{Na}$ and $\mathrm{Au}$, concentrations of some relatively volatile elements in the Vigarano and Leoville coarse-grained inclusions of this study are not distinctly lower than those of Allende coarse-grained inclusions. For example, mean $\mathrm{Cl}$ chondrite-normalized enrichment factors for $\mathrm{Ni}(0.22 \pm 0.12)$, $\mathrm{Co}(0.06 \pm 0.02), \mathrm{Cr}(0.18 \pm 0.03)$, and $\mathrm{Mn}(0.03 \pm 0.02)$ in the Vigarano and Leoville coarse-grained inclusions are greater than enrichment factors for these elements in about half or more of the Allende coarse-grained inclusions compiled by DAvis et al. (1982). In contrast, like $\mathrm{Na}$ and $\mathrm{Au}$, mean $\mathrm{Cl}$ chondrite-normalized enrichment factors for $\mathrm{Fe}$ $(0.031 \pm 0.005)$ and $\mathrm{Zn}(0.16 \pm 0.05)$ in the Vigarano and Leoville inclusions are at the low ends of their ranges in the Allende inclusions. In the latter, $\mathrm{Fe}$ ranges from $0.01-0.3$ $\times \mathrm{Cl}$ in thirty-four samples and is $>0.035 \times \mathrm{Cl}$ in twentythree of those samples. Zinc ranges from $0.05-0.9 \times \mathrm{Cl}$ in seventeen samples, is $>0.16 \times \mathrm{Cl}$ in twelve, and $>0.4 \times \mathrm{Cl}$ in five. GROSSMAN and GANAPATHY (1976a) showed that most Allende coarse-grained inclusions have concentrations of $\mathrm{Fe}, \mathrm{Co}$, and $\mathrm{Mn}$ that are $2 \%$ or less of those in bulk Allende. Concentrations of $\mathrm{Cr}, \mathrm{Au}$, and $\mathrm{Na}$ in those inclusions are, in contrast, $15 \%$ to more than $100 \%$ of those in bulk Allende. GROSSMAN and GANAPATHY (1976a) suggested, therefore, that if measured concentrations of $\mathrm{Fe}, \mathrm{Co}$, and $\mathrm{Mn}$ in Allende coarse-grained inclusions reflect contamination by admixed meteoritic matrix material, $\mathrm{Cr}, \mathrm{Au}$, and $\mathrm{Na}$ concentrations in those inclusions must be largely indigenous. Concentrations of $\mathrm{Cr}, \mathrm{Au}, \mathrm{Na}, \mathrm{Ni}, \mathrm{Zn}, \mathrm{Fe}, \mathrm{Co}$, and $\mathrm{Mn}$ in the four Type $B$ Vigarano and Leoville inclusions studied here are $11-17 \%$, $3-5 \%, 12-16 \%, 3-15 \%, 16-105 \%, 1-3 \%, 2-11 \%$, and $1-16 \%$, respectively, of those in their hosts, bulk Vigarano (KALLEMEYN and WASSON, 1981) or bulk Leoville (KRACHER et al., 1985). If none of the $\mathrm{Fe}$ in those inclusions is indigenous, they could have suffered as much as $1-3 \%$ matrix contamination. The amount of matrix contamination was probably much less, however, because, when we sampled these inclusions, we avoided material near inclusion/matrix interfaces. Thus, we prefer the interpretation that measured volatile element concentrations of coarse-grained inclusions in this study are largely indigenous and there is overlap of the ranges of indigenous volatile element concentrations in refractory inclusions of the reduced and oxidized subgroups of $\mathrm{C} 3 \mathrm{~V}$ chondrites, with the latter being higher for $\mathrm{Na}, \mathrm{Au}, \mathrm{Fe}$, and $\mathrm{Zn}$.

Fine-grained CAIs are thought to have been more intensely altered by reaction with low-temperature nebular gas than were coarse-grained CAls. Thus, it is not surprising that the two Vigarano fine-grained inclusions analyzed in this study, Vig 1623-14 and 1623-16, are enriched by factors of 5 and 7 in $\mathrm{Fe}, 59$ and 26 in $\mathrm{K}, 166$ and 73 in $\mathrm{Na}, 79$ and 41 in $\mathrm{Cl}$. and 24 and 23 in $\mathrm{Zn}$, respectively, compared to the mean composition of the three Vigarano Type B inclusions studied here. What is surprising is that, in contrast to coarse-grained Vigarano inclusions, fine-grained Vigarano inclusions are not strongly depleted in $\mathrm{Na}$ and $\mathrm{Au}$ compared to their counterparts in Allende. Instead, as shown in Fig. 7b, Vig 1623-14 and 1623-16 have concentrations of $\mathrm{Na}(7.14$ and $3.17 \%$, respectively) and $\mathrm{Au}$ ( $<30$ and $<5.9 \mathrm{ppb}$, respectively) that are comparable to those in nine Allende fine-grained inclusions analyzed by GROSSMAN and GANAPATHY (1975, $1976 \mathrm{~b}$ ). Concentrations of $\mathrm{Cl}$ and $\mathrm{Zn}$, two of the most volatile elements, are enriched in Vig 1623-14 by factors of 3.6 and 1.5 , respectively, and in Vig $1623-16$ by factors of 2.0 and 1.4 , respectively, relative to the mean of those nine Allende fine-grained inclusions. These comparisons suggest that $\mathrm{Vi-}$ garano fine-grained inclusions were not altered at higher temperatures or for shorter times than Allende fine-grained inclusions, as was probably the case for their coarse-grained counterparts. It is also unlikely that all fine-grained members of the reduced subgroup of $\mathrm{C} 3 \mathrm{~V}$ chondrites were altered under the same conditions. As is shown in Fig. 7b, a fine-grained Leoville inclusion, Leo 3536-1, analyzed by MAO et al. (1990) has very low concentrations of both $\mathrm{Na}$ and $\mathrm{Au}$ and a finegrained Efremovka inclusion, E14, analyzed by BorNTON et al. (1986) has a very low concentration of $\mathrm{Na}$, but not $\mathrm{Au}$.

Two inclusions studied here are not plotted in Fig. 7. One is the olivine aggregate, Vig 1623-10, which is most properly compared to Allende amoeboid olivine aggregates, and which has lower $\mathrm{Na}$ and $\mathrm{Au}$ concentrations than them. The mean of seven Allende amoeboid olivine aggregates has $2.3 \pm 0.5$ $\times \mathrm{Cl}$ chondritic $\mathrm{Na}$ and $1.4 \pm 0.3 \times \mathrm{Cl}$ chondritic $\mathrm{Au}$ (Grossman et al., 1979), whereas Vig 1623 10 has 0.33 $\pm 0.01 \times \mathrm{Cl}$ chondritic $\mathrm{Na}$ and $0.648 \pm 0.004 \times \mathrm{Cl}$ chondritic Au. Like coarse-grained inclusions, olivine aggregates of the reduced subgroup of $\mathrm{C} 3 \mathrm{~V}$ chondrites seem to have been altered at higher temperatures or for a shorter time than their counterparts in the oxidized subgroup. The other sample not plotted is the barred olivine chondrule, Vig 1623-13, which has $0.73 \pm 0.02 \times \mathrm{C} 1$ chondritic $\mathrm{Na}$ and $<0.02 \times \mathrm{C} 1$ chondritic Au. An appropriate comparison for this sample is with the mean of twenty Allende olivine chondrules given by RUBIN and WASSON (1987). The latter has $0.93 \pm 0.13$ $\times \mathrm{Cl}$ chondritic $\mathrm{Na}$ and $0.71 \pm 0.12 \times \mathrm{Cl}$ chondritic Au.

\section{CONCLUSIONS}

Six Vigarano refractory inclusions have refractory element fractionations that are rare or absent in Allende inclusions. Vig 1623-11 has Th/La and Ir/Mo ratios that are lower than those of any Allende Group I or modified Group I inclusion, and $\mathrm{Os} / \mathrm{La}, \mathrm{Ir} / \mathrm{La}$ and $\mathrm{Zr} / \mathrm{Hf}$ ratios that are among the lowest 
in those inclusions. The low Os/La, Ir/La, and Ir/Mo ratios seem to require nonrepresentative sampling of condensate phases in the nebula to a degree that is unusual for Allende Group I and modified Group I inclusions. Vig 1623-10 is an olivine aggregate with an Ir enrichment factor 1.7 times greater than the mean enrichment factor for $\mathrm{Re}, \mathrm{Os}$, and $\mathrm{Ru}$, relative to $\mathrm{Cl}$ chondrites. This is probably the result of preferential sampling of fcc relative to hcp condensate alloy phases in the nebula, a phenomenon that is not known to fractionate Ir from $\mathrm{Re}, \mathrm{Os}$, and $\mathrm{Ru}$ by more than $20 \%$ in Allende refractory inclusions or olivine aggregates. Vig 1623-13 is an olivine chondrule which has one of the lowest $\mathrm{Ir} / \mathrm{Sm}$ ratios ever measured in such an object and which probably suffered unusually severe metal/silicate fractionation before or during chondrule melting. Vig 1623-16 is a fine-grained inclusion with a modified Group II pattern formed by condensation after removal of an ultrarefractory condensate at unusually low temperatures. Vig 477-5 is a fluffy Type A inclusion with a REE pattern characterized by a small negative Ce anomaly, a large negative $\mathrm{Yb}$ anomaly, and no Eu anomaly. It probably contains two components, one removed from the nebular gas before $\mathrm{Ce}, \mathrm{Eu}$, and $\mathrm{Yb}$, the most volatile REEs, had completely condensed, and another that condensed after removal of a condensate containing all REEs except Eu. Vig 477-B is a Type $B$ inclusion with a REE pattern resembling an ultrarefractory condensate except for a sub-chondritic $\mathrm{Lu} / \mathrm{Tm}$ ratio. It is probably a mixture of a component with an ultrarefractory REE pattern and another with a modified Group II REE pattern.

MAO et al. (1990) found that three of five Vigarano and Leoville inclusions have refractory element fractionations that are rare in Allende. One has a modified Group II pattern, another has a large negative $\mathrm{Ce}$ anomaly, and a third is strongly enriched in Os compared to other refractory siderophiles, relative to $\mathrm{Cl}$ chondrites. Thus, nine of fifteen inclusions analyzed in this laboratory from the reduced subgroup of $\mathrm{C} 3 \mathrm{~V}$ chondrites have unusual or unique refractory element characteristics compared to those in Allende, a member of the oxidized subgroup. Taken together, our results seem to confirm the notion that Allende inclusions are not representative of all refractory objects that existed in the solar nebula, or even of all those sampled by $\mathrm{C} 3 \mathrm{~V}$ chondrites. In fact, LIU and SCHMITT (1988) analyzed many inclusions in Kaba and Mokoia and concluded that there are different proportions of Groups I, II, and III inclusions in each of Kaba, Mokoia, and Allende, all members of the oxidized subgroup of $\mathrm{C} 3 \mathrm{~V}$ chondrites. The fact that refractory inclusions are distributed heterogeneously even among $\mathrm{C} 3 \mathrm{~V}$ chondrites implies that nebular mixing processes were rather inefficient, at least where and when $\mathrm{C} 3 \mathrm{~V}$ chondrites accreted. This is surprising because, in order to produce the relatively unfractionated siderophile element enrichment factors of Allende inclusions such as Egg 6 (SYLVESTER et al., 1990b), rather efficient mixing of large numbers of fractionated metal alloy grains seems to be required. Inclusions such as Egg 6 may have been assembled in a rather turbulent nebular environment before being transported to a rather quiescent region, where they were incorporated into Allende without being well mixed with more fractionated inclusions like the nine found so far in $\mathrm{C} 3 \mathrm{~V}$ chondrites of the reduced subgroup.
Concentrations of $\mathrm{Na}$ and $\mathrm{Au}$ in Vigarano and Leoville coarse-grained inclusions tend to be lower than in their Allende counterparts, but this does not seem to be the case for all fine-grained inclusions. Thus, while coarse-grained inclusions of the reduced subgroup of $\mathrm{C} 3 \mathrm{~V}$ chondrites were probably altered at higher temperatures or for shorter times than those of the oxidized subgroup, some fine-grained inclusions of the reduced and oxidized subgroups may have been altered under similar conditions.

Acknowledgments-We are grateful to C. Caillet for permission to use unpublished mineral-chemical data for Leo $3537-2$ and Vig 477 B. We thank A. M. Davis and S. B. Simon for helpful discussions and T. P. Ward for laboratory assistance. This research was funded by the National Aeronautics and Space Administration through grants NAG 9-54 (L.G.) and NAG 9-230 (G.J.M.).

Editorial handling: R. A. Schmitt

\section{REFERENCES}

ANDERS E. and GREVESSE N. (1989) Abundances of the elements: Meteoritic and solar. Geochim. Cosmochim. Acta 53, 197-214.

BisCHOFF A., PALME H., and SPETTEL B. (1987) A37-a coarsegrained, volatile element-poor, $\mathrm{Ca}, \mathrm{Al}$-rich inclusion with huge Fremdlinge (abstr.). Lunar Planet. Sci. XVIII, 81-82.

Blum J. D., Wasserburg G. J., Hutcheon I. D., Beckett J. R, and STOLPER E. M. (1989) Origin of opaque assemblages in C3V meteorites: Implications for nebular and planetary processes. Geochim. Cosmochim. Acta 53, 545-558.

BOYNTON W. V. (1975) Fractionation in the solar nebula: Condensation of yttrium and the rare earth elements. Geochim. Cosmochim. Acta 39, 569-584.

BoYNTON W. V. (1978) Fractionation in the solar nebula, II. Condensation of Th, U, Pu and Cm. Earth Planet. Sci. Lett. 40, 6370.

Boynton W. V., Wark D. A., and Ulyanov A. A. (1986) Trace elements in Efremovka fine-grained inclusion E14: Evidence for high temperature, oxidizing fractionations in the solar nebula (abstr.). Lunar Planet. SCi. XVII, 78-79.

Caillet C., MacPherson G. J., and El Goresy A. (1988) Fremdinge in Vigarano CAI 477B: Assemblages, compositions and possible formational history (abstr.). Lunar Planet. Sci. XIX, 156157.

Caillet C., Zinner E. K., and MacPherson G. J. (1991) Mg-Al isotopic study of a deformed and recrystallized Leoville Type B refractory inclusion: Hot accretion into a cold matrix, and if so when? (abstr.). Lunar Planet. Sci. XXII, 167-168.

Clayton R. N., MacPherson G. J., Hutcheon I. D., Davis A. M., Grossman L., Mayeda T. K., Molini-Velsko C., and ALLEN J. M. (1984) Two forsterite-bearing FUN inclusions in the Allende meteorite. Geochim. Cosmochim. Acta 48, 535-548.

Clayton R. N., Mayeda T. K., MacPherson G. J., and GrossMAN L. (1987) Oxygen and silicon isotopes in inclusions and chondrules from Vigarano (abstr.). Lunar Planet. Sci. XVIII, 185186.

CONARD R. (1976) A study of the chemical composition of Ca-Alrich inclusions from the Allende meteorite. M.S. thesis, Oregon State University.

DAvis A. M. and Grossman L. (1979) Condensation and fractionation of rare earths in the solar nebula. Geochim. Cosmochim. Acta 43, 1611-1632.

Davis A. M., Grossman L., and Allen J. M. (1978) Major and trace element chemistry of separated fragments from a hibonitebearing Allende inclusion. Proc. 9th Lunar Planet. Sci. Conf., 12351247.

Davis A. M., TANaka T., Grossman L., LeE T., and Wasserburg G. J. (1982) Chemical composition of HAL, an isotopically unusual Allende inclusion. Geochim. Cosmochim. Acta 46, 1627-1651.

Davis A. M., MACPherson G. J., and Hinton R. W. (1986) Rims 
revealed-ion microprobe analysis of individual rim layers in a Vigarano Type A inclusion (abstr.). Meteoritics 21, 349-351.

DAvis A. M., HINTON R. W., and MACPHERSON G. J. (1987) Relict grains in a Vigarano refractory inclusion (abstr.). Meteoritics 22, 363-365.

Davis A. M., MACPHERSON G. J., Clayton R. N., MAYeda T. K., SYlvester P. J., Grossiman L., Hinton R. W., and LaUGHLiN J. R. (1991) Melt solidification and late-stage evaporation in the evolution of a FUN inclusion from the Vigarano $\mathrm{C} 3 \mathrm{~V}$ chondrite. Geochim. Cosmochim. Acta 55, 621-637.

Ekambaram V., Kawabe I., TanaKa T., Davis A. M., and GrossMAN L. (1984) Chemical compositions of refractory inclusions in the Murchison $\mathrm{C} 2$ chondrite. Geochim. Cosmochim. Acta 48, 20892105.

El Goresy A., CAllet C., and ZinNer E. (1990a) Vigarano CAI 477B: Petrography and $\mathrm{Mg}$ isotopic composition of the core and individual rim layers (abstr.). Lunar Planet. Sci. XXI, 321-322.

El. GORESY A., KIMURA M., and ZINNER E. (1990b) Isotopic and trace element study of ALH85085 inclusions (abstr.). Meteoritics 25, 360-361.

Fahfy A. J., Goswami J. N., McKeegan K. D., and Zinner E. $(1987){ }^{26} \mathrm{Al},{ }^{244} \mathrm{Pu},{ }^{50} \mathrm{Ti}, \mathrm{REE}$ and trace element abundances in hibonite grains from $\mathrm{CM}$ and $\mathrm{CV}$ meteorites. Geochim. Cosmochim. Acta 51, 329-350.

FEGLeY B., JR., and PALME H. (1985) Evidence for oxidizing conditions in the solar nebula from Mo and $W$ depletions in refractory inclusions in carbonaceous chondrites. Earth Planet. Sci. Lett. 72, 311-326.

GrossmaN J. N. and WASSON J. T. (1983) Refractory precursor components of Semarkona chondrules and the fractionation of refractory elements among chondrites. Geochim. Cosmochim. Acta 47, 759-771.

GROSSMAN J. N. and WASSON J. T. (1985) The origin and history of the metal and sulfide components of chondrules. Geochim. Cosmochim. Acta 49, 925-940.

Grossman J. N., Rubin A. E., Rambaldi E. R., Rajan R. S., and Wasson J. T. (1985) Chondrules in the Qingzhen type-3 enstatite chondrite: Possible precursor components and comparison to ordinary chondrite chondrules. Geochim. Cosmochim. Acta 49, 17811795.

GrosSMAN L. (1972) Condensation in the primitive solar nebula. Geochim. Cosmochim. Acta 36, 597-619.

GrossmaN L. (1973) Refractory trace elements in Ca-Al-rich inclusions in the Allende meteorite. Geochim. Cosmochim. Acta 37, 1119-1140.

GROSSMAN L. and GANAPATHY R. (1975) Volatile elements in Allende inclusions. Proc. 6th Lunar Planet. Sci. Conf., 1729-1736.

GROSSMAN L and GANAPATHY R. (1976a) Trace elements in the Allende meteorite-1. Coarse-grained, Ca-rich inclusions. Geochim. Cosmochim. Acta 40, 331-344.

GROSSMAN L. and GANAPATHY R. (1976b) Trace elements in the Allende meteorite-II. Fine-grained, Ca-rich inclusions. Geochim. Cosmochim. Acta 40, 967-977.

GrossmAN L. and STEELE I. M. (1976) Amoeboid olivine aggregates in the Allende meteorite. Geochim. Cosmochim. Acta 41, 16471664.

Grossman L., Ganapathy R., and Davis A. M. (1977) Trace elements in the Allende meteorite-III. Coarse-grained inclusions revisited. Geochim. Cosmochim. Acta 41, 1647-1664.

Grossman L., Ganapathy R., Methot R. L., and Dayis A. M. (1979) Trace elements in the Allende meteorite-IV. Amoeboid olivine aggregates. Geochim. Cosmochim. Acta 43, 817-829.

Hinton R. W., Davis A. M., SCATENA-WACHEL D. E., GROSSMAN L. and DRAUS R. J. (1988) A chemical and isotopic study of hibonite-rich refractory inclusions in primitive meteorites. Geochim. Cosmochim. Acta 52, 2573-2598.

Hutcheon I. D., PAlme H., KenNedy A., and Spettel B. (1989) Trace elements in the FUN inclusion C-1: Absence of $\mathrm{Ce}$ and $\mathrm{W}$ anomalies (abstr.). Meteoritics 24, 279.

IRELAND T. R. (1990) Presolar isotopic and chemical signatures in hibonite-bearing refractory inclusions from the Murchison carbonaceous chondrite. Geochim. Cosmochim. Acta 54, 3219-3237.

IRELAND T. R. and FEGLEY B. (1991) REE abundance patterns in
CAI's: A new classification (abstr.). Lunar Planet. Sci. XX1I, 611612.

IRELAND T. R., FAHEY A. J., and ZiNNER E. K. (1988) Trace-element abundances in hibonites from the Murchison carbonaceous chondrite: Constraints on high-temperature processes in the solar nebula. Geochim. Cosmochim. Acta 52, 2841-2854.

KallemeYN G. W. and WASSON J. T. (1981) The compositional classification of chondrites-I. The carbonaceous chondrite groups. Geochim. Cosmochim. Acta 45, 1217-1230.

KaWABE I., TANaka T., EKambaram V., Davis A. M., and GrossMAN L. (1986) INAA determination of holmium in submilligram samples of cosmochemical and geochemical interest and the second-order activation interference. J. Radioanal. Nucl. Chem. 102, $227-238$.

Kracher A., Kell K., Kallemeyn G. W., Wasson J. T., Clayton R. N., and Huss G. I. (I985) The Leoville (CV3) accretionary breccia. Proc. I6th Lunar Planet. Sci. Conf;; Part I, J. Geophys. Res. 90, (supplement), DI23-D135.

Kuehner S. M., Laughlin J. R., Grossman L., Johnson M. L., and BURNETT D. S. (1989) Determination of trace element mineral/liquid partition coefficients in melilite and diopside by ion and electron microprobe techniques. Geochim. Cosmochim. Acta 53, 3115-3130.

IIU Y.G. and SCHMITT R. A. (1988) The relative nebular locations for the formation of parental components in the CV3 chondrites Kaba, Mokoia and Allende (abstr.). Lunar Planet. Sci. XIX, 684685.

LIU Y.-G., RAJAN R. S., and SCHMITT R. A. (1987) Mokoia Ca-Al inclusions (CAl's) with negative and positive $\mathrm{Ce}$ anomalies-interim report \#2 (abstr.). Lunar Planet. Sci. XVIII, 562-563.

Loss R. D. Lugmair G. W. MacPherson G. J., and Davis A. M. (1990a) Isotope anomalies in Vigarano CAl's-Hic et ubique (abstr.). Lunar Planet. Sci. XXI, 718-719.

Loss R. D., Lugmair G. W., MacPherson G. J., and Davis A. M. (1990b) Isotopically distinct reservoirs in the early solar system (abstr.). Meteoritics 25, 380-381.

MACPHERSON G. J. (1985) Vigarano refractory inclusions: Allende unaltered, and a possible link with $\mathrm{C} 2$ inclusions (abstr.). Meteoritics 20, 703-704.

MACPherson G. J. and DAvis A. M. (1990) A petrologic and ion microprobe study of a Vigarano Type B2 refractory inclusion: Evolution by multistage melting and recrystallization following alteration (abstr.). Meteoritics 25, 382.

MACPHERSON G. J. and GROSSMAN L. (1981) A once-molten, coarsegrained, Ca-rich inclusion in Allende. Earth Planet. Sci. Lett. 52, 16-24.

MacPherson G. J, and Grossman L. (1984) "Fluffy" Type A $\mathrm{Ca}, \mathrm{Al}$-rich inclusions in the Allende meteorite. Geochim. Cos mochim. Acta 48, 29-46.

MacPherson G. J., Grossman L., Allen J. M., and BecketT J. R. (1981) Origin of rims on coarse-grained inclusions in the Allende meteorite. Proc. 12th Lunar Planet. Sci. Conf., 1079-1091.

MacPherson G. J., Davis A. M., and Grossman J. N. (1989) Refractory inclusions in the unique chondrite ALH85085 (abstr.). Meteoritics 24, 297.

Mao X.-Y., Ward B. J., Grossman L., and MacPherson G. J. (1990) Chemical compositions of refractory inclusions from the Vigarano and Leoville carbonaceous chondrites. Geochim. Cosmochim. Acta 54, 2121-2132.

Mao X.-Y., WARD B. J., Grossman L., and KECK B. D. (1991) Chemical composition of $T-21$ silica tubing and implications for neutron activation analysis. J. Radioanal. Nucl. Chem. 149, 97108 .

MASON B. and MARTIN P. M. (1977) Geochemical differences among components of the Allende meteorite. Smithsonian Contrib. Earth Sci. 19, 84-95.

MASON B. and TAYLOR S. R. (1982) Inclusions in the Allende meteorite. Smithsonian Contrib. Earth Sci. 25, 1-30.

MCSWEEN H. Y., JR. (1977) Petrographic variations among carbonaceous chondrites of the Vigarano type. Geochim. Cosmochim. Acta 41, 1777-1790.

MISAWA K. and NAKAMURA N. (1988) Rare earth elements in chon- 
drules and CAI from the Felix (CO3) chondrite (abstr.). Lunar Planet. Sci. XIX, 784-785.

Nagasawa H., Blanchard D. P., Jacobs J. W., Brannon J. C., PHILPOTTS J. A., and ONUMA N. (1977) Trace element distribution in mineral separates of the Allende inclusions and their genetic implications. Geochim. Cosmochim. Acta 41, 1587-1600.

PALME H. and WlotZKA F. (1976) A metal particle from a Ca-Alrich inclusion from the meteorite Allende, and condensation of refractory siderophile elements. Earth Planet. Sci. Lett. 33, 4560.

Palme H., Wlotzka F., Nagel K., and El Goresy A. (1982) An ultra-refractory inclusion from the Ornans carbonaceous chondrite. Earth Planet. Sci. Lett. 61, 1-12.

PALME H., LARIMER J. W., and LIPSCHUTZ M. E. (1988) Moderately volatile elements. In Meteorites and the Early Solar System (eds. J. F. KERRIDGE and M. S. MATTHEWS), pp. 436-461. University of Arizona Press.

Pernicka E. and Wasson J. T. (1987) Ru, Rc, Os, Pt and Au in iron meteorites. Geochim. Cosmochim. Acta 51, 1717-1726.

PodoseK F. A., ZinNer E. K., MacPherson G. J., LUNDberg L. L., BRANnun J. C., and FaheY A. J. (1991) Correlated study of initial ${ }^{87} \mathrm{Sr} /{ }^{86} \mathrm{Sr}$ and $\mathrm{Al}-\mathrm{Mg}$ isotopic systematics and petrologic properties in a suite of refractory inclusions from the Allende meteorite. Geochim. Cosmochim. Acta 55, 1083-1110.

RUBIN A. E. and WASSON J. T. (1987) Chondrules, matrix and coarsegrained chondrule rims in the Allende meteorite: Origin, interrelationships and possible precursor components. Geochim. Cosmochim. Acta 51, 1923-1937.

RUBIN A. E. and WASSON J. T. (1988) Chondrules and matrix in the Ornans $\mathrm{CO} 3$ meteorite: Possible precursor components. Geochim. Cosmochim. Acta 52, 425-432.

SimON S. B., GroSSMAN L., and DAvis A. M. (1991) Fassaite composition trends during crystallization of Allende Type B refractory inclusion melts. Geochim. Cosmochim. Acta 55, 2635-2655.

SYLVESTER P. J., Grossman L., and MACPHERSON G. J. (1990a) Chemical compositions of Leoville and Vigarano inclusions (abstr.). Lunar Planet. Sci. XXI, 1231-1232.

Sylvester P. J., WARD B. J., Grossman L., and Hutcheon I. D. (1990b) Chemical compositions of siderophile element-rich opaque assemblages in an Allende inclusion. Geochim. Cosmochim. Acta 54, 3491-3508.

WARK D. A. and LOVERING J. F. (1977) Marker events in the early solar system: Evidence from rims on Ca-Al-rich inclusions in carbonaceous chondrites. Proc. 8th Lunar Planet. Sci. Conf., 95-112.

WARK D. A. and LOVERING J. F. (1982) The nature and origin of type $\mathrm{Bl}$ and $\mathrm{B} 2 \mathrm{Ca}-\mathrm{Al}$-rich inclusions in the Allende meteorite. Geochim. Cosmochim. Acta 46, 2581-2594.

Wark D. A., Boynton W. V., Keays R. R., and Palme H. (1987) Trace element and petrologic clues to the formation of forsteritebearing Ca-Al-rich inclusions in the Allende meteorite. Geochim. Cosmochim. Acta 51, 607-622.

WASSON J. T., OUYANG X., WANG J., and JERDE E. (1989) Chemical classification of iron meteorites: XI. Multi-element studies of 38 new irons and the high abundance of ungrouped irons from Antarctica. Geochim. Cosmochim. Acta 53, 735-744.

ZinNer E. K., CAILleT C., and El Goresy A. (1989) Mg- and O-isotopic compositions of periclase, spinel and melilite from Vigarano CAI 477B (abstr.). Lunar Planet. Sci. XX, 1245-1246. 